\title{
Use of protein immobilization to measure cytochrome P450 conduction and metabolism kinetics
}

Christopher D. Bostick

Follow this and additional works at: https://researchrepository.wvu.edu/etd

\section{Recommended Citation}

Bostick, Christopher D., "Use of protein immobilization to measure cytochrome P450 conduction and metabolism kinetics" (2015). Graduate Theses, Dissertations, and Problem Reports. 5238.

https://researchrepository.wvu.edu/etd/5238

This Dissertation is protected by copyright and/or related rights. It has been brought to you by the The Research Repository @ WVU with permission from the rights-holder(s). You are free to use this Dissertation in any way that is permitted by the copyright and related rights legislation that applies to your use. For other uses you must obtain permission from the rights-holder(s) directly, unless additional rights are indicated by a Creative Commons license in the record and/ or on the work itself. This Dissertation has been accepted for inclusion in WVU Graduate Theses, Dissertations, and Problem Reports collection by an authorized administrator of The Research Repository @ WVU.

For more information, please contact researchrepository@mail.wvu.edu. 


\title{
USE OF PROTEIN IMMOBILIZATION TO MEASURE CYTOCHROME P450 CONDUCTION AND METABOLISM KINETICS
}

\author{
Christopher D. Bostick \\ Dissertation submitted to the School of Pharmacy \\ at West Virginia University \\ in partial fulfillment of the requirements \\ for the degree of \\ Doctor of Philosophy \\ in \\ Pharmaceutical and Pharmacological Sciences \\ Grazyna Szklarz, Ph.D., Chair \\ David Lederman, Ph.D. \\ Yon Rojanasakul, Ph.D. \\ William Petros, Pharm.D., FCCP \\ Tim Tracy, Ph.D., RPh \\ Department of Pharmaceutical Sciences \\ Morgantown, West Virginia \\ 2015
}

Keywords: Cytochrome P450, Drug Metabolism, Protein Immobilization, Protein-Protein Interactions, Protein Electron Transport, Nanoscale Platform, Conductive Probe Atomic Force Microscopy, Molecular Modeling

Copyright 2015 Christopher D. Bostick 


\title{
Abstract
}

\section{USE OF PROTEIN IMMOBILIZATION TO MEASURE CYTOCHROME P450 METABOLISM KINETICS AND ITS EFFECTORS}

\author{
by Christopher D. Bostick
}

\begin{abstract}
Cytochrome P450s (P450s) are a large family $(>11,000)$ of heme thiolated proteins that are responsible for $\sim 75 \%$ of the metabolism of pharmaceuticals on the market. Understanding P450 mediated metabolism is crucial for accurate in vitro predictions of drug metabolism. P450 protein-protein interactions have been shown to alter enzyme catalytic activity. Furthermore, these interactions are isoform specific, and can elicit activation, inhibition, or no effect on enzymatic activity. Studies show these effects are also dependent on the protein binding partner cytochrome $\mathrm{P} 450$ reductase (CPR), and the order of protein addition to purified reconstituted enzyme systems. In the current work, we use controlled immobilization of P450s to a gold surface to gain a better understanding of P450-P450 interactions between three key drugmetabolizing isoforms (CYP2C9, CYP3A4, and CYP2D6). Molecular modeling was used to assess the favorability of homo/heteromeric P450 complex formation. P450 complex formation in vitro was analyzed in real-time utilizing surface plasmon resonance (SPR). Lastly, the effects of P450 complex formation were investigated utilizing our immobilized platform and reconstituted enzyme systems.
\end{abstract}

Molecular modeling shows favorable binding of CYP2C9-CPR, CYP2C9-CYP2D6, CYP2C9CYP2C9, and CYP2C9-CYP3A4 in rank order. $\mathrm{K}_{\mathrm{D}}$ values obtained via SPR show strong binding, in the nanomolar range, of the above pairs, with CYP2D6 yielding the lowest $\mathrm{K}_{\mathrm{D}}$, followed by CYP2C9, CPR, and CYP3A4. Metabolic incubations show immobilized CYP2C9 metabolism was activated by homomeric complex formation. CYP2C9 metabolism was not affected by the presence of CYP3A4 with saturating CPR concentrations. CYP2C9 metabolism was activated by CYP2D6 in solution, but inhibited when CYP2C9 was immobilized, both at saturating and sub-saturating CPR concentrations. Order of addition of proteins (CYP2C9, CYP2D6, CYP3A4, and CPR) influenced magnitude of inhibition for CYP3A4, but not CYP2D6. These results indicate isoform specific P450 interactions and effects on P450 mediatedmetabolism. These findings are important in evaluating how in vitro results are obtained for measuring P450 kinetics, and provide a better mechanistic understanding of P450-P450 interactions to allow for better prediction of in vivo metabolism from in vitro data.

We also demonstrate that gold nanopillars, functionalized with an organic self-assembled monolayer, can be used to measure the electrical conductance properties of immobilized P450s without aggregation. Given that transfer of the $1^{\text {st }}$ electron to the P450 heme group acts as the gating step for the catalytic cycle, understanding electron transfer in P450s could shed light on metabolism kinetics. Conductance measurements of nanopillars with immobilized CYP2C9 using conducting probe atomic force microscopy demonstrate that a correlation exists between the energy barrier height between hopping sites and CYP2C9 metabolic activity. Measurements performed as a function of tip force indicate that, when subjected to a large force, the protein is more stable in the presence of a substrate. This agrees with the hypothesis that substrate entry into the active site helps to stabilize the enzyme.

The relative distance between hopping sites also increases with increasing force, possibly because protein functional groups responsible for electron transport depend on the structure of 
the protein. The inhibitor sulfaphenazole, in addition to the previously studied aniline, increased the barrier height for electron transfer and thereby makes CYP2C9 reduction more difficult and inhibits metabolism. This suggests that P450 Type II ligands may decrease the ease of electron transport processes in the enzyme, in addition to occupying the active site. These findings further our understanding of how P450 metabolism is mediated through substrates, and provides an important technological advancement for studying P450s that avoids complications found in current methodologies. These two studies demonstrate the ability of an immobilized P450 platform to provide information on protein-protein interactions, substrate protein interactions, and atypical enzyme kinetics. 


\section{Dedication}

I dedicate this to my mother Debbie Bostick, who obtained her first college degree while raising me and working two jobs. It is from her that I learned the most important ideal one can have is to never give up on anything you desire, no matter how daunting the task. 


\section{Acknowledgements}

The process of obtaining a PhD is both intellectually demanding and emotionally draining. It is for this reason I owe a great deal of thanks to the many people who have helped ease both of these aspects during my journey.

I would first like to thank my advisor Dr. Peter Gannett who has always had an open door and ready ear during my PhD. There was never a time I could not rely on your help during my studies, and even after your departure from WVU, you still remained involved and interested in how I was doing both in research and personally. I couldn't envision a better advisor to learn from, and feel I have learned a great deal as your mentee.

Thank you to my Co-advisor Dr. David Lederman for helping me broaden my knowledge outside of my field of expertise. You pushed me to think critically about my research and to understand each step of what I was doing, and I am a better scientist today because of it. I would like to thank Dr. Grazyna Szklarz who stepped in to be my advisor upon Dr. Gannett's departure. All of the assistance you gave me during the hardest time of my PhD was invaluable. I would also like to recognize the rest of my committee, Dr. Timothy S. Tracy whose skyped with me many times to help me be a better pharmacokinetics guy, Dr. Yon Rojanasakul for his expertise in nanoparticles, and Dr. William Petros for his valued in vivo pharmacology experience. I have always felt prepared answering questions at conferences due to the quality of the ones I received in my committee meetings.

I owe a great deal of gratitude to all the members of the lab past and present who have been like brothers and sisters in arms. My research would not be possible without the willingness of my colleagues to share their time and expertise with me. I thank Dr. Katherine Hickey, Emily Despeaux, Dr. Lance Wollenberg, Dr. John Jett, Dr. Brian Train, Dr. Darcy Flora, and Dr. Robyn Ayscue. I would also like to thank Wesley Tish, Andrew Biundo, and Emma Dolan, the undergraduate students who I mentored that were always eager to learn. I also thank the Dr. Lederman lab group for sharing their techniques and knowledge with me to help me develop my research.

I like to give special thanks to the staff of the School of Pharmacy for going above beyond in helping students such as myself, and getting to know me on a personal level. I would especially like to recognize Penny Daily, Anna Rittenhouse, Donna Mathess, and Amy Newton.

I would also like to thank the staff of the shared research facilities who have devoted countless hours training me, helping me with research design, and inevitably fixing my mistakes. Thank you Dr. Kolin Brown, Dr. Harley Hart, Dr. Weiqiang Ding. Dr. Marcela Redigolo, and Dr. Huiyuan Li.

I thank Kim Quedado for being an excellent fellowship manager who really cared about all of us as individuals. No matter how big or small the need Kim gave it her all to help, whether it was something I needed for research or a conference I wanted to attend.

I give many thanks to family and friends who have put up with me being gone for so long to get my $\mathrm{PhD}$, and who have offered me reprieve from research from time to time. 
Last, but not least, I thank Lindsay Lueptow for being a constant source of joy during some of my hardest times. Your constant encouragement has kept me going when I didn't think I could, and I'm happier for having you in my life. 


\section{Table of Contents}

1. Introduction: Understanding Cytochrome P450 Mediated Metabolism and its Effectors ........1

1.1. P450 Function and Mechanism of Action .............................................................

1.2. P450-P450 Interactions Effect on Metabolism ............................................... 11

1.3. Applications of Immobilized Protein Platforms .............................................. 13

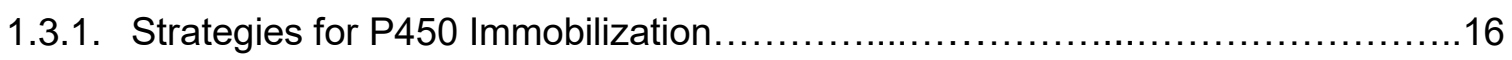

1.3.2. Characterization of Immobilized P450s.................................22

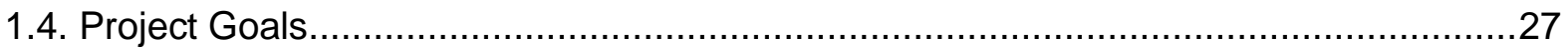

2. Immobilized Cytochrome P450 for monitoring of P450-P450 Interactions and Metabolism..31

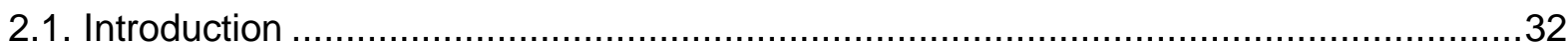

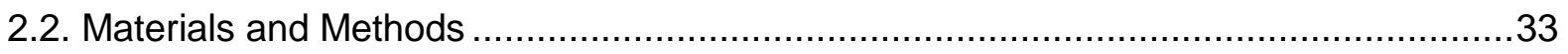

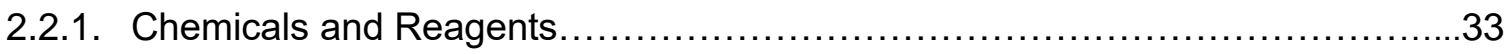

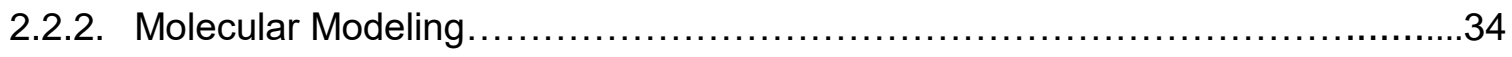

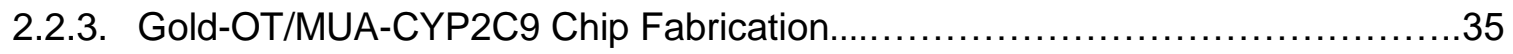

2.2.4. Surface Plasmon Resonance Binding Analysis............................

2.2.5. Immobilization of CYP2C9 Enzyme on Gold/Silicon Platform...................36

2.2.6. Reconstituted CYP2C9 Enzyme Incubation...................................

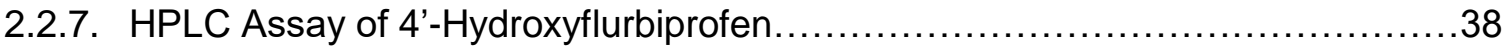

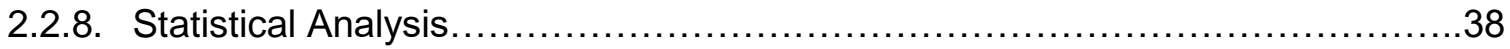

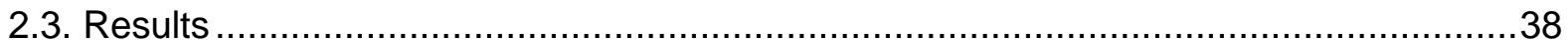

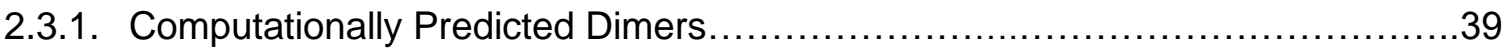

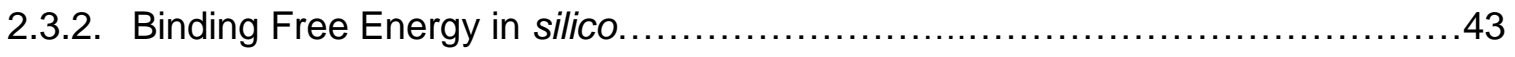

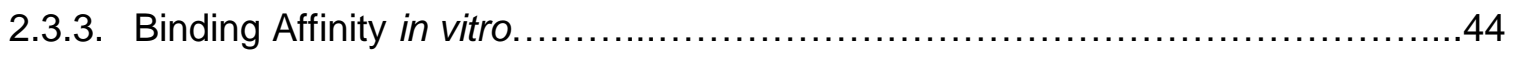

2.3.4. CYP2C9 effect on CYP2C9i-Mediated Flurbiprofen Metabolism...............46 
2.3.5. Effect of CYP3A4 and CYP2D6 on CYP2C9-mediated metabolism of

flurbiprofen

2.3.6. Influence of the order of addition of enzymes on CYP2C9-mediated

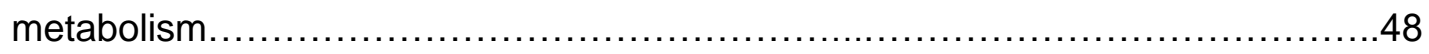

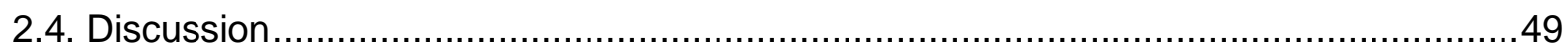

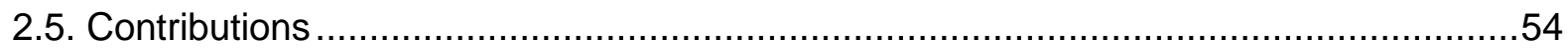

3. Nanoscale electron transport measurements of immobilized cytochrome P450 proteins .....55

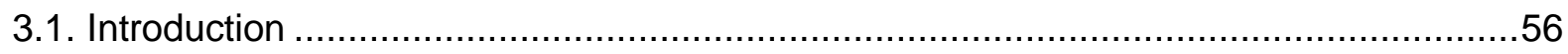

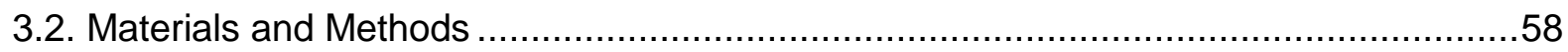

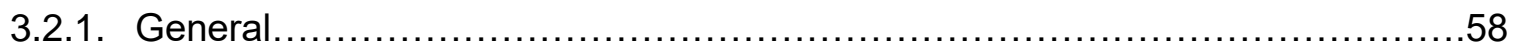

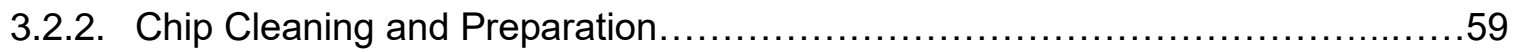

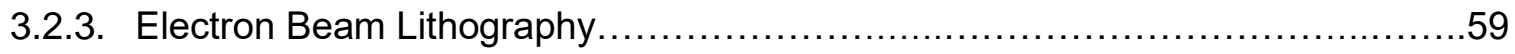

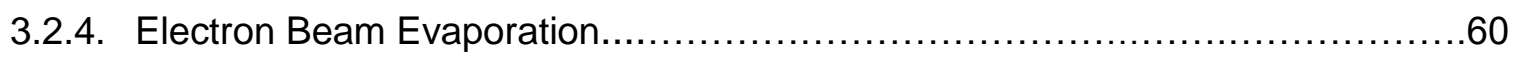

3.2.5. Scanning Electron Microscopy and Atomic Force Microscopy Imaging............60

3.2.6. Immobilization of CYP2C9 to Gold via Self Assembled Monolayer..................60

3.2.7. Conductive Probe Atomic Force Microscopy Measurements.....................61

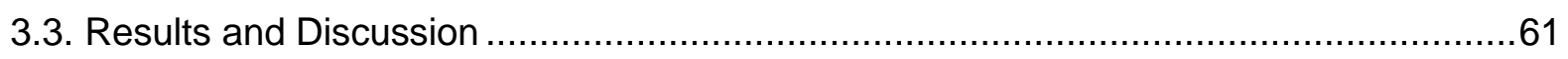

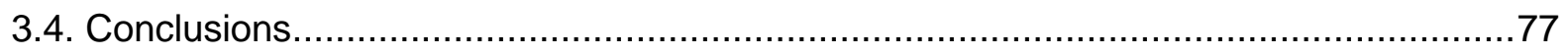

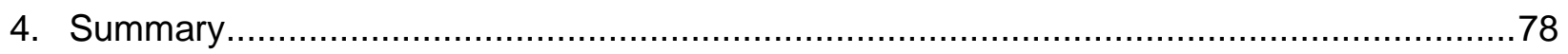

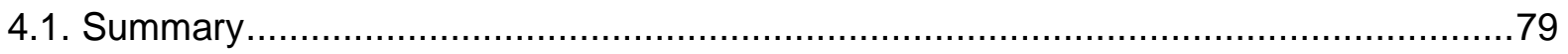

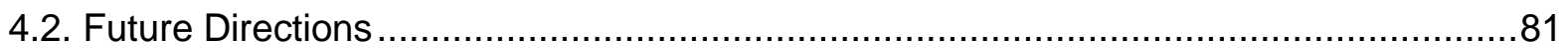

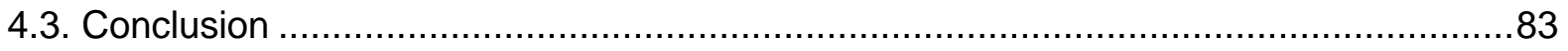

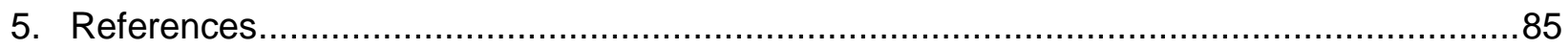

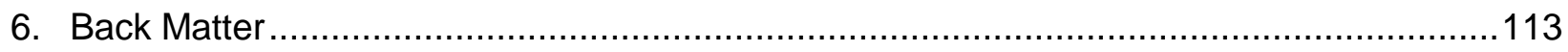




\section{List of Figures}

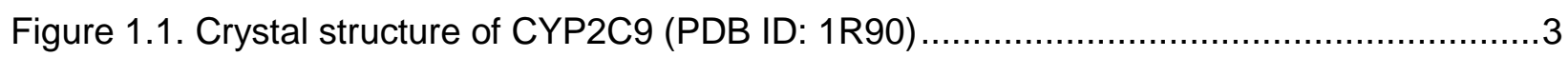

Figure 1.2. Cytochrome P450 catalytic cycle diagram ............................................... 4

Figure 1.3. Cartoon representation of $\mathrm{P} 450$ and $\mathrm{CPR}$ in the smooth endoplasmic reticulum .......5

Figure 1.4. Plot detailing Michaelis-Menten enzyme kinetics ...........................................10

Figure 1.5. Lineweaver-Burk plots of different types of reversible enzyme inhibition ...............11

Figure 1.6. Schematic of P450 adsorbed to a bare gold electrode for mediatorless reduction

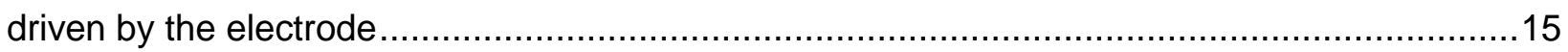

Figure 1.7. Cartoon representation of enzyme immobilization on different surfaces ................17

Figure 1.8. Diagram of Surface Plasmon Resonance Spectroscopy ...............................25

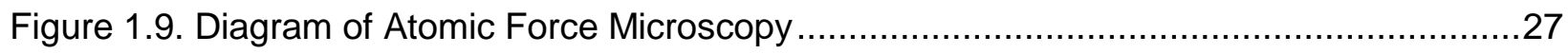

Figure 2.1. Representation of the top scored interaction predicted by GRAMMX for 1 R9O bound

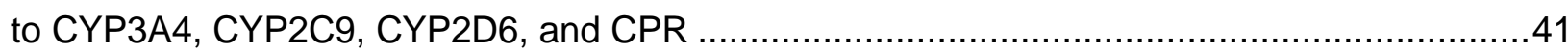

Figure 2.2. Relative arrangements of CYP2C9 (1R9O or 1OG5) and partner P450s (CYP2C9,

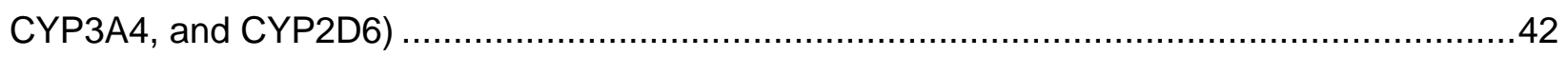

Figure 2.3. Scheme for CYP2C9 coupling to self-assembled monolayer of thiols...................44

Figure 2.4. Representative sensorgram of CYP2C9 immobilization ..................................45

Figure 2.5. SPR sensorgram obtained from system comprised of soluble CYP2D6 binding to

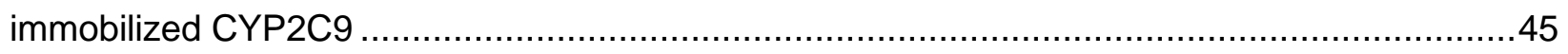

Figure 2.6. Metabolite production by immobilized CYP2C9 in the presence of soluble CYP2C9. 
Figure 2.7. Metabolite production in CYP2C9-CYP3A4 system

Figure 2.8. Metabolite production in CYP2C9-CYP2D6 system 48

Figure 2.9. Order of addition of CPR and CYP3A4 or CYP2D6 effect on CYP2C9 mediated flurbiprofen metabolism

Figure 3.1. SEM image of fabricated gold nanopillars 63

Figure 3.2. AFM tapping mode images of gold coated with SAM and SAM/CYP2C9 .64

Figure 3.3. Height distributions of pillars before and after CYP2C9 functionalization 65

Figure 3.4. Measured I-V curves of immobilized CYP2C9 as a function of force 66

Figure 3.5. CYP2C9 substrates used in CPAFM studies

Figure 3.6. I-V curves for CYP2C9 alone and in the presence of flurbiprofen and dapsone or sulfaphenazole.

Figure 3.7. Measured I-V curves of CYP2C9 and substrates on different sessions showing reproducibility. 68

Figure 3.8. Poole-Frenkel plot for the data for positive bias voltages for nanopillars 71

Figure 3.9. Poole-Frenkel plot for the data obtained for positive bias voltages for nanopillars .73

Figure 3.10. Poole-Frenkel plot for I-V curves taken at $32 \mathrm{nN}$ on Pillar $\mathrm{A}$ .74

Figure 3.11. Change in barrier height and barrier width as a function of force for pillars. .75

Figure 3.12. Simplified depiction of electron transfer through hopping sites within a protein immobilized to gold when uncompressed and compressed. 76 


\section{List of Tables}

Table 2.1. CYP2C9 regions of interest and the relevant residues

Table 2.2. Dimer Binding Sites Predicted by GRAMMX ....................................................40

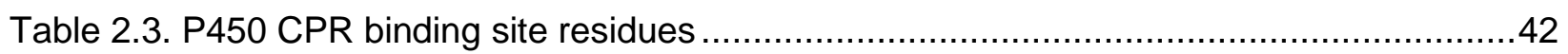

Table 2.4. Planar alignment of CPR binding site with dimer partner molecule ..........................43

Table 2.5. MM-GBSA Binding Free Energies for CYP2C9 Dimers ........................................44

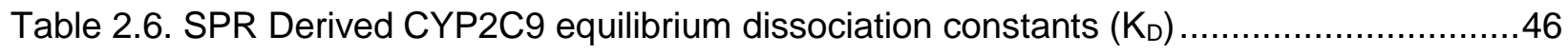

Table 3.1. Results of the Fits to CYP2C9 I-V data with the Poole-Frenkel model ......................72 


\section{Abbreviations}

BOE, Buffered Oxide Etch; CD, Circular Dichroism; CPAFM, Conductive Probe Atomic Force Microscopy; CTAB, Cetyltrimethylammonium Bromide; b5, Cytochrome b5; P450, Cytochrome P450; CYP2C9, Cytochrome P450 2C9; CYP2D6, Cytochrome P450 2D6; CYP3A4, Cytochrome P450 3A4; CPR, Cytochrome P450 Reductase; Dap, dapsone; DDAB, didodecyldimethylammonium bromide; DMPC, dimeristoyl-I-a-phosphatidylcholine; DLPE, dilauroylphosphatidylethanolamine; DSPE, distearoylphosphatidylethanolamine; DLPC, Dilauroylphosphatidylcholine; EDC, N-(3-dimethylaminopropyl)-N'-ethylcarbodiimide Hydrochloride; EC-STM, Electrochemical scanning tunneling microscopy; ET, Electron Transfer; ETp, Electron transport; EPG, edge-plane pyrolytic graphite; FAD, flavin adenine dinucleotide; Flurbi, flurbiprofen; FMN, flavin mononucleotide; GCE, glassy carbon electrodes; $\mathrm{CL}_{\text {int, }}$ intrinsic clearance; KPI, Potassium Phosphate Buffer $\mathrm{pH}=7$; LbL, layer-by-layer; MM/GBSA, Molecular Mechanics/Generalized Born Surface Area; MM/PBSA, Molecular Mechanics/Poisson Boltzmann Surface Area; MUA, 11-Mercaptoundecanoic Acid; NHS, NADPH, nicotinamide adenine dinucleotide phosphate; NHE, normal hydrogen electrode; N-hydroxysulfosuccinimide Sodium Salt; OT, 1-Octanethiol; PBS, Phosphate Buffered Saline; PMMA, PEI, poly(ethyleneimine); Poly(methyl methacrylate); PF, Poole-Frenkel; QCM, quartz crystal microbalance; RT, Room Temperature; SAM, Self-Assembled Monolayer; SCE, saturated calomel electrode; SEM, Scanning Electron Microscope; SERRS, surfaced enhanced resonance raman spectroscopy; STM, scanning tunneling microscopy; SPR, Surface Plasmon Resonance; yCPR, yeast CPR; $V_{\max }$, maximum velocity WT, wild-type 
Chapter 1

\section{Introduction: Understanding Cytochrome P450 Mediated Metabolism and its Effectors}




\subsection{P450 Function and Mechanism of Action}

P450 catalyzed reactions were first described for the metabolism of the azo dye 4dimethylaminoazobenzene by liver homogenates in the $1940 \mathrm{~s}^{1}$, but the identity of the enzyme responsible was unknown. Twenty years later this enzyme was identified when a unique spectral peak was observed with a maxima at $450 \mathrm{~nm}$ when the protein was reduced by dithionite and bound to carbon monoxide ${ }^{2}$. This spectral signature is where P450s derive their name. A study in 1968 revealed a redox partner was necessary for the P450 mediated $\omega$ hydroxylation of fatty acids $^{3}$, which would later be identified as cytochrome P450 reductase (CPR). Expression of P450s was achieved first in human liver microsomes, but gave rise to low yields ${ }^{4}$. Later scientific advancements would allow expression of recombinant human P450s in Escherichia coli, with much greater yields ${ }^{5}$. The ability to isolate purified protein allowed extensive characterization including generation of high resolution crystal structures of $\mathrm{P} 450 \mathrm{~s}^{6}$, as well as the functional interaction between P450s and $\mathrm{CPR}^{7}$.

A nomenclature system was developed based on genetic similarity to identify the 14 P450 families, and 26 subfamilies found in mammals ${ }^{8,9}$. P450s are designated as CYPs, denoting cytochrome P450. CYP is followed by a number placing it in a gene family, which share $\geq 40 \%$ genetic sequence identity. Next is a letter that denotes a sub family, which share $\geq 55 \%$ genetic sequence identity. Lastly, each individual gene receives a second number. Thus the name CYP2C9 would represent a cytochrome P450 in the '2' family, the 'C' subfamily, and individual gene ' 9 '. In addition, there also exist allelic variants of P450 isoforms resulting from single nucleotide polymorphisms altering the amino acid sequence. These mutant CYPs are labeled by the isoform followed by an * and a number greater than ' 1 ', with ' 1 ' being reserved for the wild type. Expression of these mutants occurs more frequently in some populations, and has been demonstrated to affect catalytic activity, as evidenced by difference in warfarin hydroxylation by CYP2C9*3 compared to wild type CYP2C $9^{*} 1^{10}$. 
P450s constitute of a large family $(>11,000)$ of heme containing proteins. In humans the P450 enzymes are found primarily in the liver where they are membrane bound in the smooth endoplasmic reticulum ${ }^{11}$. The main function of P450s is to take lipid-soluble substrates and convert them to more water soluble products by insertion of an oxygen atom and the creation of a hydroxylated product. Of the 57 P450 genes identified by the human genome project, 15 found in the liver are principally responsible for metabolizing xenobiotic agents such as drugs; 20 participate in the biosynthesis of steroids, sterols, fatty acids, and eicosanoids; 7 play key roles in sterol biosynthesis; and 15 are enzymes whose functions still remain unknown ${ }^{12}$. P450s are responsible for the metabolism of $75 \%$ of all pharmaceuticals on the market ${ }^{13}$. Furthermore, five P450 isoforms, CYP1A2, CYP2C19, CYP2C9, CYP2D6, and CYP3A4, are responsible for 95\% of P450-mediated metabolism in humans ${ }^{14}$.

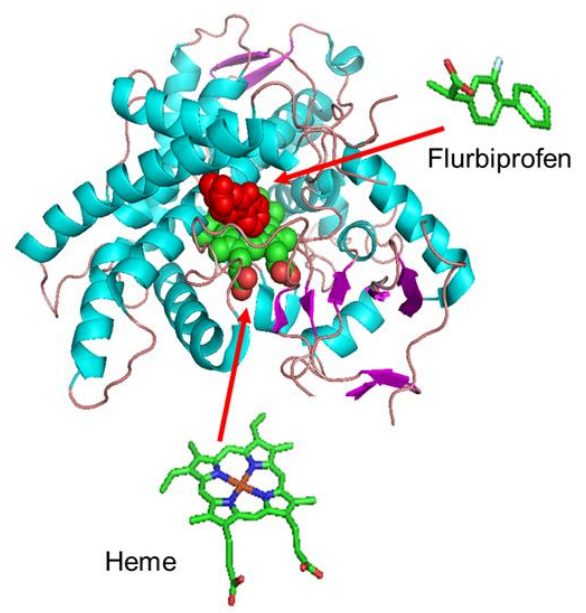

Figure 1.1 Crystal structure of CYP2C9 with bound flurbiprofen (1R9O.pdb). CYP2C9 displayed in ribbon diagram with heme group and flurbiprofen depicted in space filling model, and outside of the active site as stick figure models. Figure rendered in Pymol (www.pymol.org). 
P450s contain a heme group within the active site composed of an iron atom coordinated in a porphyrin ring (Figure 1.1). A series of reductions and oxidations must occur to allow the splitting of a dioxygen and hydroxylation of a substrate. Looking at Figure 1.2 we can identify the important steps of the catalytic cycle as such, i) binding of substrate in the active site with the heme iron in the low spin, +3 or ferric state $(1 \rightarrow 2)$, ii) donation of an electron by the binding partner CPR (2) results in the formation of the +2 ferrous iron to which oxygen then binds (3), iii) donation of a second electron from CPR (4), iv) cleavage of the dioxygen occurs and there is a loss of water and formation of the perferryl species (5), v) abstraction of the hydrogen atom from the substrate followed by the radical recombination step $(6 \rightarrow 7)$, and finally, vi) the hydroxylated substrate (metabolite) exits from the active site (8) and concludes the cycle ${ }^{15}$ returning the enzyme to its initial state. Of central importance to the catalytic cycle is the transfer of two electrons from nicotinamide adenine dinucleotide phosphate (NADPH), in two distinct steps, via CPR to the P450 heme.

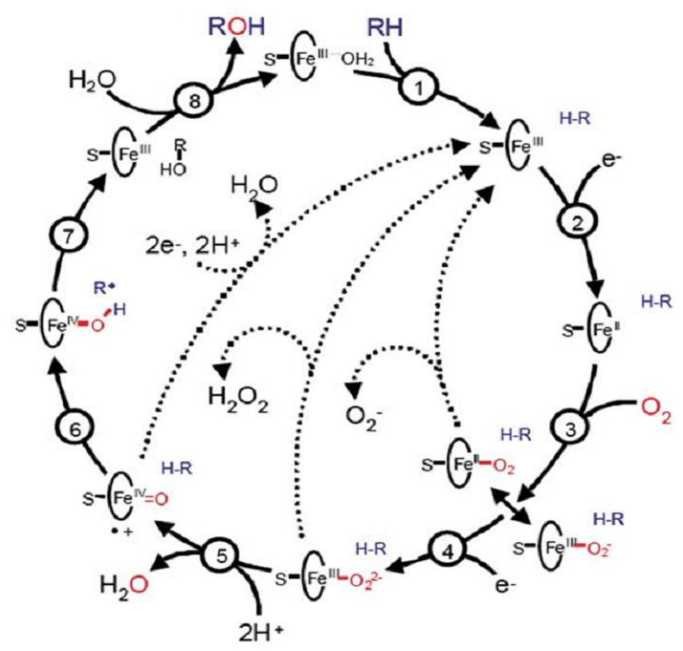

Figure 1.2. Catalytic cycle of a Cytochrome P450 enzyme. Electrons transferred in step two is provided by CPR, and electron transferred in step four is provided by CPR or cytochrome b5. Dotted lines in the interior of the diagram represent shunt pathways that prevent $P 450$ from metabolizing itself in the event of substrate exit prior to the finish of the catalytic cycle. Reproduced in part from reference ${ }^{16}$ with permission of The Royal Society of Chemistry. 
P450s are membrane bound via their hydrophobic N-terminus, and are co-localized with protein partners CPR and $b_{5}$ (Figure 1.3) ${ }^{11}$. Their mechanism of action is dependent upon on the sequential transfer of two electrons. The first transfer can only be accomplished by cytochrome P450 reductase $(\mathrm{CPR})$, and the second transfer requires CPR or cytochrome $\mathrm{b}_{5}(\mathrm{~b} 5)^{16}$. While CPR is required for P450 metabolism, b5 is not required for P450-mediated metabolism, and its presence, in certain instances, simply activates metabolism ${ }^{17-19}$.

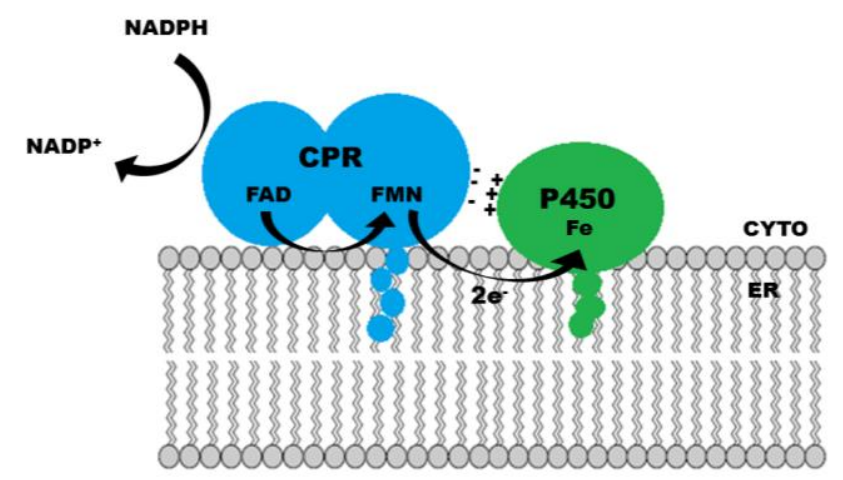

Figure 1.3. Cartoon representation of the smooth $E R$ membrane containing a P450 and CPR. CPR and P450 interact through the shown electrostatic interactions. The electron transfer pathway is shown by the arrows.

CPR contains four regions including a flavin mononucleotide (FMN), a flavin adenine dinucleotide (FAD) prosthetic groups, a connecting flexible hinge domain, and a NADPH-binding domain ${ }^{20}$. FAD accepts a hydride ion from NADPH, and reduced FAD donates electrons to FMN, which, in turn, transfers electrons to the heme center of cytochrome P45021. Transfer from CPR to the heme is mediated by a binding location on the proximal side of $P 450 s^{7,22}$ (substrate binds on the distal side). CPR is able to open and close about its flexible hinge domain, brining both prosthetic groups in close proximity, allowing the transfer of electrons ${ }^{23}$. FRET studies have shown that when closed CPR it is not able to interact with P450, but is in the optimal configuration to be reduced. Reduction causes opening, exposing the FMN residues that participate in P450 binding ${ }^{24}$. In microsomes, P450 concentration exceed those of CPR by at least five fold ${ }^{25,26}$ suggesting enzyme mobility may be required ${ }^{27,28}$, or multiple P450s utilize a 
single CPR for the supply of electrons from NADPH ${ }^{29-31}$. The exact interplay of CPR and P450 in vivo has yet to be fully elucidated and this needs to be taken into consideration during in vitro study analysis.

P450s are able to oxidize (metabolize) a wide variety of substrates resulting in various reactions including carbon hydroxylation, heteroatom oxygenation, $\mathrm{N}$ - and $\mathrm{O}$ - dealkylation, epoxidation of olefins, aromatic oxidation, heteroatom oxidation, aldehyde oxidations, and dehydrogenation of organic molecules ${ }^{32,33}$. The insertion of oxygen by P450 enzymes into the chemical structure of many xenobiotics can often create reactive or otherwise toxic metabolites $^{34}$ that subsequently undergo chemical reactions. "Reactivity" of metabolites is characterized by their stability (or lack thereof), and how far they can travel before they are inactivated through, for example, covalent binding ${ }^{35}$. There are stable metabolites that exit the organ of generation, long-lived that can exit the tissue of origin, intermediate-lived that exit the cell of origin but not the tissue, short-lived that diffuse away from the enzyme but are not able to leave the cell, and ultra short-lived that form covalent adducts at the site of generation. Reactive species that can result from P450 bioactivation include free radicals and electrophilic metabolites. These compounds are able to bind or bond to proteins ${ }^{36}$ or DNA ${ }^{37}$, and are able to cause mitochondrial permeability transition pore opening ${ }^{38}$, hepatotoxicity ${ }^{39}$, carcinogenesis $^{40}$, and increased immune hypersensitivity ${ }^{41}$. In addition, highly reactive metabolites may act as mechanism based inactivators of P450s through alkylation of the heme group, as is seen in CYP3A4 and substrate raloxifene ${ }^{42,43}$. This is of importance for drug development as reactive metabolites can not only cause toxicity, but also create drug-drug interactions by inactivation of P450s.

Another issue to consider is the possibility of drug-drug interactions that occur through substrate effects with various pharmaceutical agents. The heme group in P450s is known to have a low and high spin state that can correlate with metabolism rate. Type I substrates are 
known to induce a high spin state upon binding to the heme, while Type II have no effect or stabilize the low spin state ${ }^{44}$. Many P450 inhibitors have been shown to be Type II ligands, due to their ability to stabilize the low reduction potential of P450 and prevent oxygen binding to heme ${ }^{45}$. However, there are many substrates such as dapsone that do not alter the spin state but are still metabolized, thus there must be other alterations made that facilitate electron transfer and metabolism. A study by Guengerich and Johnson ${ }^{16}$ showed that the reduction of heme occurred in the absence of substrate in some P450s, and equal reduction rates between low and high spin heme were shown. Another proof of this can be seen in a single enzyme study by our lab measuring electron transport though CYP2C9, that showed no difference in ease of electron transport between flurbiprofen and dapsone despite known opposite effects on spin state of the two substrates ${ }^{46}$. This suggests that much is still unknown about spin states impact on metabolism, and that it may not be the best predictor for metabolism kinetics in P450 substrates.

Some P450s also display atypical kinetics due to activation, auto activation, partial inhibition, substrate inhibition, and biphasic metabolism ${ }^{47}$. The work by Korzekwa et al. ${ }^{47}$ provided a basis for multiple binding sites within a P450. This was later proven by Nuclear Magnetic Resonance studies done by our lab on CYP2C9, showing that flurbiprofen and dapsone can occupy the active site simultaneously ${ }^{48}$. This allows some substrates to act allosterically as effectors, altering metabolic rates for other substrates. For example, dapsone has been shown to be an activator of CYP2C9 mediated flurbiprofen metabolism through shifting flurbiprofen into a more favorable position for metabolism in the active site ${ }^{17}$. Furthermore, testosterone has been shown to act as its own effector for CYP3A4, with up to two or three able to fit in the active site simultaneously ${ }^{49}$. Currently identification of these pairs or triplets requires time intensive metabolism studies ${ }^{50}$. The possibility of multiple binding site model and allosteric modulation is also still under debate ${ }^{51}$. 
Given the large percentage of pharmaceuticals metabolized by P450s, many efforts have gone into effective ways to study their metabolism in vitro. The two widely used assays are solution based systems with either human liver microsomes or purified reconstituted systems. Human liver microsomes are purified through ultracentrifugation of homogenized liver tissue ${ }^{52}$. These are useful tools for early drug screening as they contain all the P450s and protein binding partners found in vivo. However, the presence of multiple P450 isoforms prevents easy determination of metabolism by specific isoforms, and more in depth studies using isoform specific inhibitors are generally needed to parse out P450s primarily involved in metabolism ${ }^{53,54}$. For these reasons drug-drug interactions, and protein-protein effects can be difficult to predict with these systems. Another approach is the use of reconstituted systems created by adding single recombinant P450 isoforms, charged lipids, and protein partners to form a microsomal structure $^{55-57}$. Control of the isoform type, substrates, and protein partners allows interrogation of substrate and protein interactions and their effects on metabolism kinetics.

Using these in vitro solution assays, we are able to study P450 kinetics in vitro utilizing mathematical models based on the rate of metabolite formation. A simplified model for P450 enzymatic substrate conversion is the Michaelis-Menten model:

$$
E+S \underset{K_{-1}}{\stackrel{K_{1}}{\Longrightarrow}}[E S] \stackrel{K_{2}}{\Longrightarrow} E+P
$$

In this model $S$ represents the substrate, $E$ represents the enzyme, ES represents the substrate-enzyme complex, and $P$ represents product of enzymatic conversion of the substrate. In this model all reactions are, in principle, reversible, and thus enzymatic conversion must occur while enzyme and substrate are complexed. The rate of the reaction is described by the kinetic rates above and below the reaction arrows. $K_{1}$ denotes the rate of formation for the enzyme-substrate complex, while $K_{-1}$ is the dissociation of the substrate enzyme complex without enzymatic conversion. Lastly, $K_{2}$ represents the rate of enzymatic conversion of 
substrate into metabolite $(P)$ by the enzyme, and is usually the rate limiting step. From this model the Michaelis-Menten equation can be derived:

$$
V=\frac{V_{\max }[S]}{K_{m}+[S]}
$$

In this equation $V$ is the velocity of the reaction, $S$ represents the concentration of substrate, and $K_{m}$ represents the Michaelis constant, the substrate concentration where half the maximum velocity $\left(V_{\max }\right)$ is achieved. This equation results in a hyperbolic graph when velocity is plotted against substrate concentration (Figure 1.4). Use of this equation requires the steady state assumption and rapid equilibrium conditions, where the enzyme-substrate complex reaches a non-changing concentration quickly or $\mathrm{d}[\mathrm{ES}] / \mathrm{dt}=0 . K_{m}$ is often used as a measure of affinity as it is equal to $\left(K_{-1}+k_{2}\right) / k_{1}$, and thus gives insight on binding of substrate to an enzyme for conversion. Michaelis-Menten kinetics are a saturable process, meaning as the substrate concentration increases the $V_{\max }$ can be calculated using the rate limiting step $K_{2}$ (also known as the turnover rate) (Equation 1.3).

$$
V_{\text {max }}=k_{2}[E]_{\text {tot }}
$$




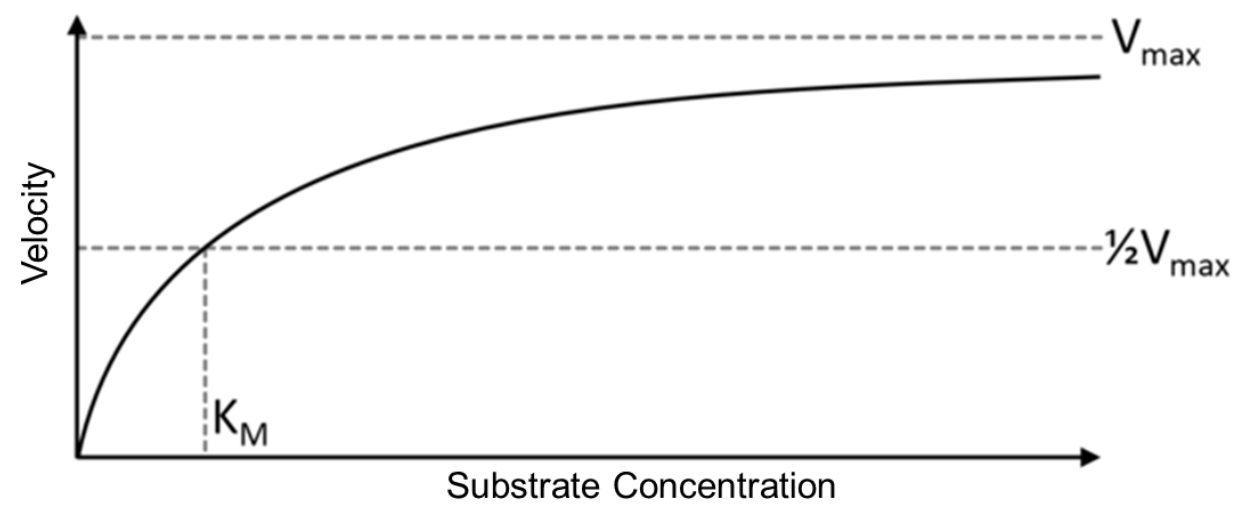

Figure 1.4. Plot detailing Michaelis-Mentin kinetics commonly observed in P450 mediated metabolism. Velocity is generally represented as metabolite concentration/time/P450 concentration.

The kinetic parameters $K_{m}$ and $V_{\max }$ have important implications in drug discovery and development, mainly for correlating in vitro kinetics to in vivo drug clearance. The intrinsic clearance $\left(C L_{\text {int }}\right)$ of a substrate is analogous to the in vivo intrinsic clearance, and is not influenced by physiological determinants such as blood flow or drug binding within the matrix ${ }^{58}$. Assuming in vivo that substrate concentration is less than $10 \%$ of $K_{m}$ (a typical assumption) and that the velocity equals the product of $C L_{\text {int }}$ and substrate concentration, we can obtain an equation for clearance as shown below:

$$
C L_{\text {int }}=\frac{V_{\max }}{K_{m}}=\frac{V}{[S]}
$$

Determination of $C L_{\text {int }}$ is thus the method for making in vitro - in vivo correlations of drug clearance to allow estimations for "first dose in man" and in vivo drug disposition. This equation also shows the importance of the kinetic constants $V_{\max }$ and $K_{m}$, and how they are crucial in making these in vitro predictions. Given the importance of these constants, it is essential to monitor how substrate interactions and protein interactions affect their values to limit otherwise unforeseen substrate toxicity. Different types of inhibition for instance are known to shift $V_{\max }$ and $K_{m}$ values in different ways, and this information can be obtained from in vitro analysis using graphical evaluation through different types of reciprocal plots ${ }^{59-61}$ (Figure 1.5). 


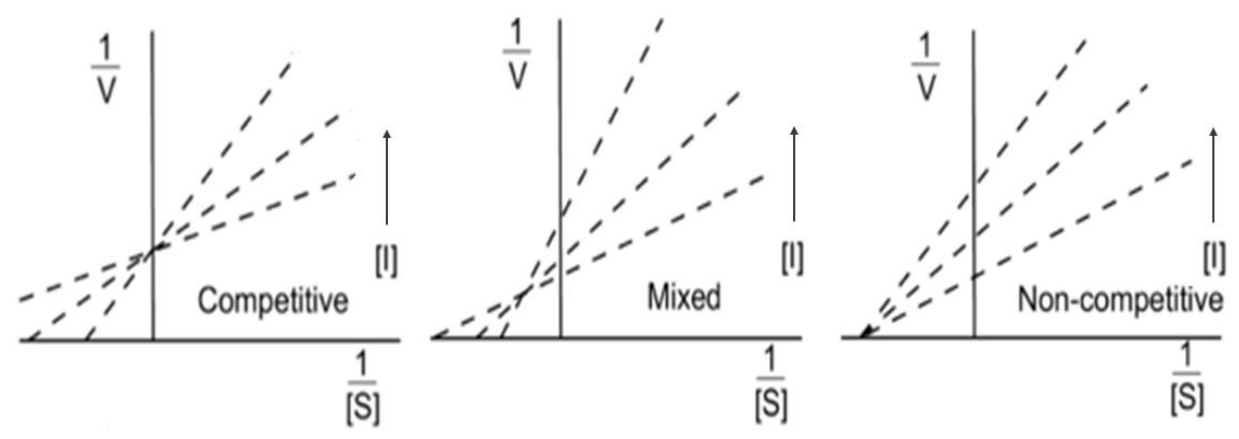

Figure 1.5. Lineweaver-Burk plots of different types of reversible enzyme inhibition. The Lineweaver-Burk plot is a double reciprocal plot of the Michaelis-Menten equation that can be used to calculate $K_{m}$ and $V_{\max }$. In these plots $S$ represents the substrate concentration and I represents the inhibitor concentration. The $y$-intercept equals $1 / V_{\max }$, and the $x$-intercept represents $-1 / K_{m}$. Another use of these plots is quick determination of the type of enzyme inhibition based on changes in $K_{m}$ and $V_{\text {max. }}$. In competitive inhibition the $K_{m}$ increases while the $V_{\max }$ is unchanged, in mixed inhibition the $K_{m}$ increases and $V_{\max }$ decreases, and in non-competitive inhibition the $K_{m}$ is unchanged and the $V_{\max }$ decreases.

\subsection{P450-P450 Interaction Effects on Metabolism}

Protein-protein interactions with P450s have been observed in multiple microsomal and reconstituted systems. P450s have been shown to form homo- and heterooligomers in solution $^{62,63}$, and a range of effects from these interactions can occur. Certain P450s can interact with each other and alter catalytic rate ${ }^{64-66}$. In addition, studies have shown that these interactions and effects are isoform dependent ${ }^{67,68}$. In order to better understand P450 metabolism, many studies have investigated the nature of these P450-P450 interactions, and their implications for in vitro metabolism predictions.

One of the early steps to understand P450-P450 interactions was to determine the mechanism by which they occur. It was proposed that P450s interact with each other through electrostatic and/or hydrophobic interactions and thus researchers sought to use detergents to disrupt possible binding. Studies using the detergent Emulgen 913 showed that CYP2B4 could be brought to monomeric form and that there were alterations (increases) in both $V_{\max }$ and $K_{m}{ }^{69,70}$. In contrast, CYP1A2 was found to require much higher concentrations of Emulgen 913 
to produce monomeric forms, and at the higher detergent concentrations, a loss of monooxygenase activity was seen ${ }^{71}$. However, non-ionic detergents were later shown to modulate P450 metabolism at concentrations that would produce no changes in aggregation state of the $\mathrm{P}_{450^{72}}$ and thus confound interpretation of data from prior studies utilizing detergents.

To investigate the role of electrostatic interactions on P450-P450 binding, studies as a function of ionic strength were also investigated. One study showed that CYP1A2 inhibition of CYP2B4 mediated 7-pentoxyresorufin-O-dealkylation was alleviated with increasing ionic strength buffers ${ }^{73}$. Another study showed that synergism of 7-pentoxyresorufin-O-dealkylation and 7-ethoxyresorufin-Odeethylation production by CYP1A2- CYP2E1 complexes at subsaturating CPR was eliminated at increasing ionic strengths ${ }^{74}$. However, it has been shown that CPR electron transfer is increased in high ionic strength buffers ${ }^{75}$, and this could supress the effect of the P450-P450 interaction. The above results do suggest that P450 heteromeric complex formation alters metabolism kinetics of one or both binding partners.

Several studies have demonstrated that the $\mathrm{N}$-terminus could play a part in aggregation and it has been reported that modifications of the $\mathrm{N}$-terminus are necessary to isolate $\mathrm{P} 450 \mathrm{~s}$ for crystallization ${ }^{76}$. In addition, in vitro studies have shown that the $\mathrm{N}$-terminus is necessary for CYP2C8 dimerization ${ }^{77}$ and the formation of a CYP2C9-CYP3A4 heteromeric complex ${ }^{78}$. Fluoresence studies also show that the N-terminus is necessary for association of CYP2C2 and CYP2C1 in the endoplasmic retiuculum of living cells ${ }^{79,80}$. However, other studies have shown that aggregation of some P450 isoforms is independent of the $\mathrm{N}$-terminus. Thus, these results taken together suggest that the role of the $\mathrm{N}$-terminus in $\mathrm{P} 450$ interactions is isoform dependent.

There are three mechanisms by which $\mathrm{P} 450-\mathrm{P} 450$ interactions are proposed to affect catalytic rate of substrate metabolism ${ }^{81}$. In the first mechanism, there is no formation of a P450P450 complex, and effects are from competition for available CPR. In this case, the binding 
affinity of CPR to P450 would govern the reaction rate, and effects could be alleviated by increased concentration of CPR. The second mechanism consists of the formation of a P450P450 complex that alters the binding affinity of the P450s for CPR. In this case, conformational changes of the P450s brought about by complex formation may alter CPR affinity and all complex formation rates have to be considered in CPR affinity (i.e. binding of CPR to the individual P450s and binding of CPR to the complexed P450s). Lastly, formation of a P450P450 complex alters the substrate turnover rate for one or both of the P450s. Again, in this case, the affect of P450 complexation is dependent upon how the isolated P450 metabolic rate compares to that of the complexed P450. Unlike the second mechanism, this response would be seen at both subsaturating and saturating CPR concentrations. This substrate turnover could be brought about by changes in electron transfer (ET) from CPR to the P450 heme group through conformational changes.

As P450 aggregation occurs more in solution, aggregation effects will be overestimated in reconstituted systems giving inaccurate in vivo predictions from in vitro results. Thus far it is not understood how oligomer formation alters metabolism, and the effect could be due to a variety of factors including conformational changes and alterations in P450 substrate turnover. Another important factor is how CPR interacts with monomeric P450s compared to homo/heteromeric P450 complexes. There is a lack of information on how CPR binding is changed by aggregation of P450s, and how ET would occur. As noted earlier, attempts to prevent P450 aggregation have also been shown to alter P450 metabolism (independent of aggregation affects) and thus confound interpretation of metabolism kinetics. In order to fully understand P450 metabolism, it is necessary to render them in a manner that mimics the in vivo membrane bound environment.

\subsection{Applications of Immobilized Protein Platforms}

Proteins have generally evolved over time into structures capable of precise reactions including highly-specific substrate recognition, analyte binding, and facile and directional 
electron tunneling ${ }^{82}$. In addition, many reactions such as such as photosynthesis, respiration, water oxidation, molecular oxygen reduction, and nitrogen fixation are performed by metalloproteins, which account for nearly half of all proteins found in nature ${ }^{83,84}$. Due to their ability to catalyze a vast amount of reactions, there has been much interest in harnessing their abilities for downstream applications. One desired application is biocatalysis in synthetic organic chemistry. The high chemo-, regio-, and stereoselectivity of enzymes negates the need for protecting groups, and thus reduces synthetic steps and alleviates work flow ${ }^{85}$. This is of special interest to the pharmaceutical industry as bioreactors, a vessel were chemical reactions are carried out by biological components, can efficiently accomplish large scale production of fine chemicals $^{86-90}$, including metabolites of P450 mediated reactions. Another use of biocatalysis is bioremediation, or the removal of toxic or hazardous substances by naturally occurring organisms ${ }^{91}$.

The high specificity of proteins also makes them ideal candidates for use as biosensors. In this case they are immobilized to a solid support and act as transducers of an optical ${ }^{92,93}$ or electronic signal that correlates with protein-analyte interaction ${ }^{94}$. Optimization of the solid support has demonstrated that these biosensors can work with low voltage potentials, have high sensitivities, exhibit low detection limits, and offer long-term stability for measurements ${ }^{95-97}$. These biosensors are currently being developed for use in drug monitoring ${ }^{98}$, environmental toxin detection ${ }^{99,100}$, and early disease detection ${ }^{101,102}$.

Two studies in 1977 demonstrated the ability of direct electron transfer from an electrode to the redox active heme group of cytochrome $\mathrm{c}^{103,104}$. This is known as mediatorless reduction, as it obviates the need of electron donors (NADPH) and protein partner (CPR) (Figure 1.6). In addition to these studies, works by Scheller et al. ${ }^{105}$ demonstrated electrochemically driven cytochrome P450 hydroxylation without the presence of NADPH. This ability has been used to improve biosensors by eliminating the need for costly reagents ${ }^{106}$ and for the creation of bioelectronics devices that exploit proteins unique properties. Using organic molecules as 
electronic components, first proposed in $1974^{107}$, has been suggested as a possible solution to the physical limitations of micro- and nano-scale electronic development. This is an attractive idea because proteins are small (nanoscale) and capable of chemomechanical, electromechanical, optomechanical, and optoelectronic processes ${ }^{108}$, making them ideal candidates for electronic components. Research has sought to use

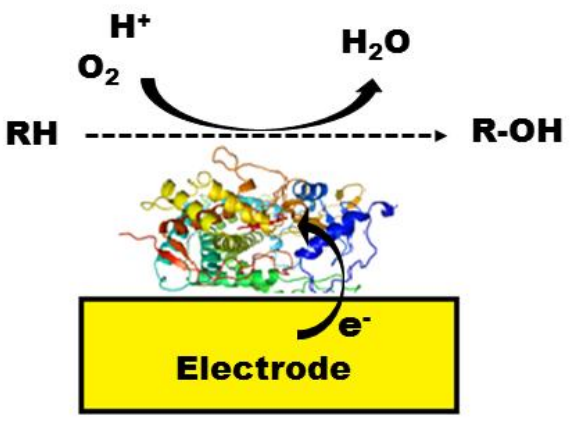

Figure 1.6. Schematic of $P 450$ adsorbed to a bare gold electrode for mediatorless reduction driven by the electrode in place of NADPH and CPR. In some cases the adsorbed $P 450$ retains metabolic activity, and upon heme reduction can hydroxylate substrate (dashed line).

proteins in the design of bioelectronics devices including field effect transistors for sensing ${ }^{109,110}$, bio-molecular transistors for data storage ${ }^{111,112}$, bio-molecular diodes ${ }^{113}$, bio-hybrid solar cells ${ }^{114}$, bio-computers ${ }^{115}$, and enzymatic biofuel cells ${ }^{116-118}$. There is also an extensive biomedical application for devices such as implantable sensors for monitoring chemicals ${ }^{119,120}$ and the creation of artificial retinas ${ }^{109}$ and noses ${ }^{121}$.

Generally, useful properties of proteins are granted by their prosthetic groups, including the metal center of metalloproteins such as P450s, and chromophores of photoactive proteins such as bacteriorhodopsin ${ }^{122}$. It has also been well demonstrated that the electrical/conductive properties of proteins are reliant on prosthetic groups, as evidenced by differences in electron transport (ETp) observed between holo- and apoazurin ${ }^{123}$. Recently, scientists have sought to enhance and optimize use of these proteins through immobilization to various solid state materials. The ability to immobilize proteins allows for direct study of electrical properties, including ETp ${ }^{124}$. ETp is defined as the travel of electrons across/through the entire protein, while ET is travel of electrons from electronically localized donor and acceptor domains. This is of interest not only for bioelectronics, but also because a proteins ETp characteristics have been shown to be correlated to its ET and redox potential ${ }^{125,126}$.

In order to successfully incorporate proteins into bioelectronics and utilize them, a deep 
understanding of electron transfer is crucial. Thus there has been extensive research into electron transfer in proteins ${ }^{124,127}$ and the development of some well characterized model systems such as Cytochrome $c^{128}$. In contrast, there have been fewer studies on ET in P450s. Given that ET acts as the gating step for the P450 catalytic cycle, enhanced understanding could shed light on P450 function/mechanism and aid in using P450s as biosensors for personal drug monitoring ${ }^{129}$, bioreactors in transformations/metabolic engineering ${ }^{87}$, and bioremediation ${ }^{91}$.

Protein immobilization is a powerful tool that is capable of controlling protein aggregation and allowing single molecule analysis. However, it is important to consider the effects of immobilization on protein structure and function. In order for immobilized proteins to be useful it is important that they retain biologic activity and reaction specificity. Several factors that determine protein function after immobilization are amino acid composition of the enzyme surface, physical and chemical properties of the solid state support, and the kind of interface between the protein and the solid state support ${ }^{130}$. All of these factors play into the structure, orientation, and conformation of the immobilized protein.

\subsubsection{Strategies for P450 Immobilization}

The first strategy employed for P450 immobilization was direct absorption to a bare electrode. Proteins, including P450s, are generally amphiphilic with hydrophobic amino acids forming a protective shell via folding to minimize the exposure of hydrophilic amino acids to solvent. Proteins can be absorbed to surfaces through electrostatic interactions of charged surface amino acids and the solid surface ${ }^{131}$, hydrophobic interactions if there are exposed hydrophobic regions, or through tethering with a linker molecule (Figure 1.7). As suggested in Figure 1.7, direct immobilization allows poor control of orientation and can result in inactive protein conformations. It has also been shown that strong interactions can denature "soft" proteins that lack a rigid structure ${ }^{132}$. 

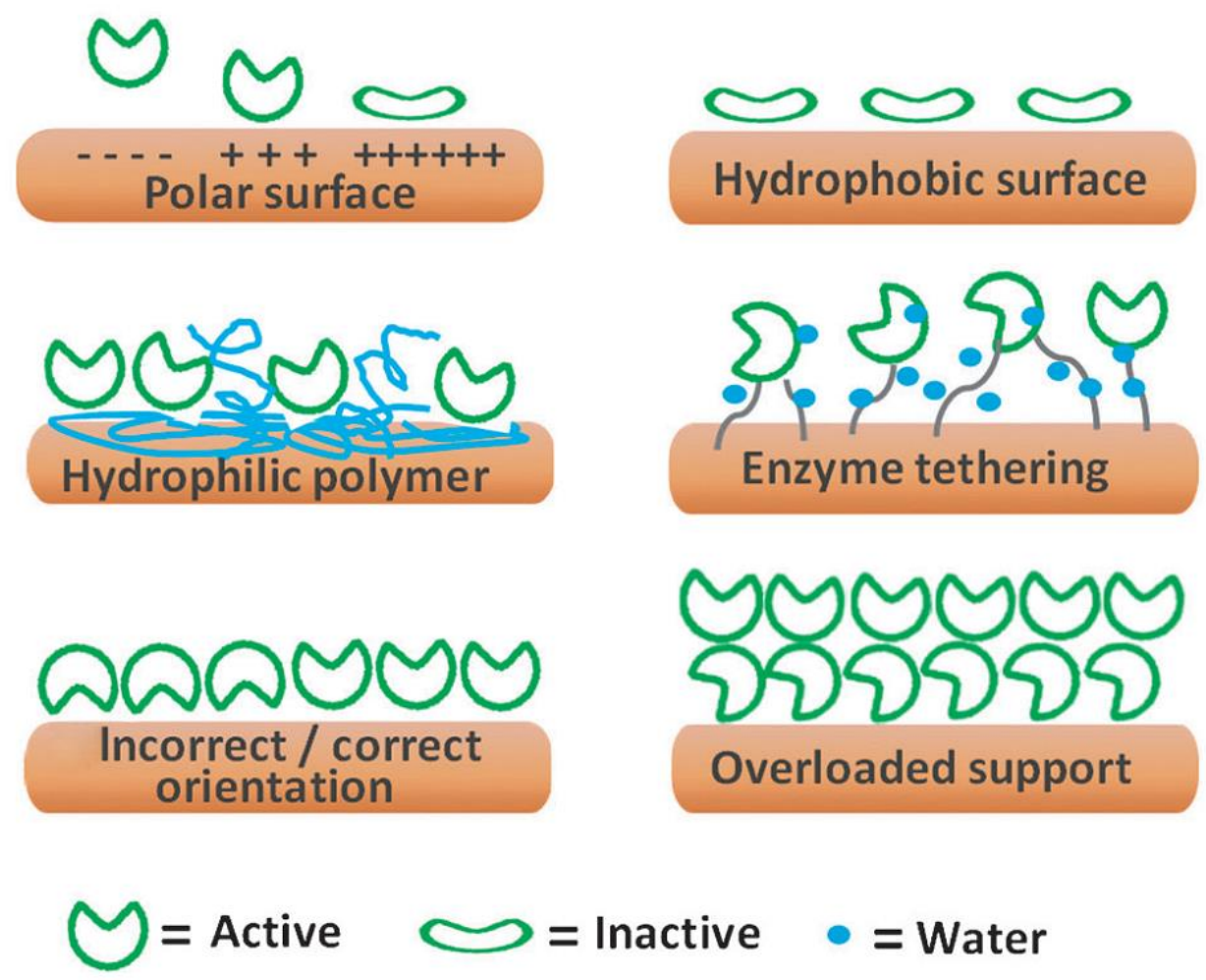

\begin{abstract}
Figure 1.7. Enzyme immobilization on different interfaces and possible effects on the enzyme conformation. High charge density (above left) or a hydrophobic surface (above right) are possible causes of enzyme conformational changes and inactivation. Enzyme co-immobilized with hydrophilic polymers (middle, left) or tethered (middle, right) can reduce unfolding and inactivating support-enzyme interactions. Incorrect orientation (below, left) and multilayer formation (below, right) may cause reduction of specific activity. Reproduced in part from reference ${ }^{131}$ with permission of The Royal Society of Chemistry.
\end{abstract}

Direct absorption of P450s was first described by Hill and coworkers for the immobilization of P450cam to edge-plane pyrolytic graphite (EPG) electrodes ${ }^{133}$. In this work, the authors propose that electrostatic interactions by positively charged amino acids (Arg-72, Arg-112, Lys344, and Arg-364) located near the proximal side and close to the heme group with the negative charges on EPG allowed for electrochemically driven catalysis yielding formal potentials ranging from -428 to $-449 \mathrm{mV}$ versus saturated calomel electrode (SCE). Further work by this group investigated the ability to achieve site-specific immobilization of P450s using surface cysteines to form a thiol bond with gold ${ }^{134}$. In these studies electrochemistry of WT P450cam was compared to mutants with all surface cysteines replaced by inert alanines (surface cysteine free, 
SCF), and a mutant containing only one surface cysteine on the proximal side (SCF-K334C) ${ }^{134}$. The results show that the single cysteine mutant yielded anodic (-50 mV vs SCE) and cathodic (-170 mV vs SCE) peaks in cyclic voltammograms, while no difference from background was observed in the WT and non-surface cysteine containing mutant. This result suggest the possibility of multiple inactive conformations of the WT P450, and that the enhanced electroactivity of SCF-K334C may be due to P450 orientation.

Direct immobilization to bare unmodified electrodes has shown to result in low electron transfer and thus limited protein activity upon immobilization ${ }^{135}$. Thus modification of the electrode surface is often used to decrease protein denaturing, enhance electron transfer, and allow control of protein orientation. Thin films have been used as one method of modifying electrode surfaces to achieve active immobilized protein. A study by Lei et al. ${ }^{136}$ used sodium montmorillonite, a member of the general mineral group of clays, to immobilize P450cam to glassy carbon electrodes (GCE). Using this immobilization method, electron transfer rates comparable to natural transfer rates from putidaredoxin to P450cam were obtained. Of note in the study is the positive shift of the redox potential of adsorbed P450cam (-361 mV vs Ag/AgCl) in comparison to $\mathrm{P} 450 \mathrm{cam}$ in solution $(-525 \mathrm{mV}$ vs $\mathrm{Ag} / \mathrm{AgCl})$. The authors hypothesize that immobilization may cause partial dehydration of P450cam, excluding water from the heme pocket. This could change the coordination of the heme iron, causing a shift from low to high $\operatorname{spin}^{137}$. Other clays, such as kaolinite, talc, goethite and orche, have also produced similar immobilization results as sodium montmorillonite ${ }^{138}$. In addition, this immobilization scheme has been shown to work for other P450 isoforms, such as CYP2B4, with the addition of Tween 80 to eliminate aggregates ${ }^{139}$.

Another notable thin film is created by modifying electrodes using lipids to mimic the lipophilic membrane environment of the smooth endoplasmic reticulum where $\mathrm{P} 450$ s reside. Lipids such as didodecyldimethylammonium bromide (DDAB), dimeristoyl-I- $\alpha$ phosphatidylcholine (DMPC), dilauroylphosphatidylethanolamine (DLPE), and 
distearoylphosphatidylethanolamine (DSPE) are able to form stable lipid bilayers through electrostatic interactions with electrodes to provide a membranous environment to facilitate electron transfer ${ }^{140}$. Studies by Shukla et al. ${ }^{141}$ found a redox potential of $-41 \mathrm{mV}$ vs normal hydrogen electrode (NHE) for CYP2C9, CYP2C18, and CYP2C19 immobilized to EPG electrodes modified with DDAB. This study however showed that only $1-3 \%$ of the immobilized P450s were electroactive, and a broad peak width (150-200 mV) was observed that exceeds the theoretical $90 \mathrm{mV}$ width for a single electron transfer, which the authors ascribe to a lack of conformational homogeneity on the surface. Of special interest in lipid bilayers is the use of Nanodisc ${ }^{\circledR}$ technology to provide a discoidal piece of membrane that is only capable of binding a single P450 enzyme, demonstrated for CYP2B4 $4^{142}$ or CYP3A4 and CPR ${ }^{143}$.

Another approach to enhance immobilization of P450s is the use of layer-by-layer (LbL) films of P450 and polyions. In LbL, multiple layers of oppositely charged films of P450s and polyions are built up on the electrode surface, providing several active layers. The ability to incorporate multiple layers on an electrode increases the effective P450 concentration, and would thus enhance downstream applications such as biosensing. Rusling and co-workers have demonstrated regular and reproducible layer formation via quartz crystal microbalance analysis with P450cam and branched poly(ethyleneimine) (PEI) on gold ${ }^{144}$. Further work demonstrated styrene epoxidation turnover on a carbon cloth electrode functionalized with multiple layers of CYP1A2 and sodium poly(styrenesulfonate) was 2.3 times faster than CYP1A2 mediated epoxidation with NADPH and CPR in solution ${ }^{145}$. Studies by Rusling and co-workers have also shown that this adsorption technique works for microsomal P450s, and that the pathway of electron transfer in this case is from the electrode to CPR and then to the P450 heme group $^{146,147}$.

Recently, the covalent attachment of P450s directly to the electrode via a tether has become a robust method to control protein adsorption. This allows for a greater degree of control of protein orientation on the surface through tailoring the choice of linker and coupling chemistry. 
This also allows for a bonded P450 that mimics their membrane attachment in vivo ${ }^{11}$. One method is to attach a molecular electronic relay to the P450 surface that allows coupled ET from the electrode to the heme group. Shumyantseva et al. ${ }^{148}$ observed electrode-driven catalysis rates comparable to NADPH-driven systems in CYP1A2 or CYP2B4 covalently attached to a flavin cofactor that was linked to rhodium-graphite electrodes. Panicco et al. ${ }^{149}$ also demonstrated the ability to measure differences in metabolism of flavin modified CYP2C9 or CYP2D6 polymorphisms using an amperometric platform. However, this method requires modification of the protein which can be difficult, and may alter protein function and conformation $^{150}$.

To overcome this problem groups have immobilized unmodified P450s through covalent attachment to self-assembled monolayers (SAM). Amphiphilic molecules, such as alkanethiols and carboxylic acids, form well-ordered mono-layers on metal and metal oxide surfaces ${ }^{151-154}$. Long chain thiols such as 11-mercaptoundecanoic acid (MUA) and hexanoic acid, and short chain thiols such as mercaptoproprionic acid and dithio-bismaleimidoethane have all been used to immobilize P450s ${ }^{155}$. It is important to consider carefully what molecule to use as a linker as length ${ }^{124,156}$ and composition ${ }^{151}$ have been shown to affect electrical properties. There are various techniques that have been used to covalently link proteins to SAMs ${ }^{157}$, with chemical activation using 1-Ethyl-3-[3-dimethylaminopropyl]carbodiimide Hydrochloride (EDC) and Nhydroxysuccinimide (NHS) being commonly used to link P450s to a carboxy-terminated SAM via surface exposed amine group.

Using EDC and NHS coupling, our group has demonstrated attachment of unmodified CYP2C9 to a mixed SAM composed of 3:1 octanethiol (OT): MUA bonded to gold ${ }^{158}$. Furthermore, the attached CYP2C9 was shown to metabolize $\Delta^{9}$-tetrahydrocannibinol (THC) to 11-hydroxy- $\triangle^{9}-\mathrm{THC}$ in the presence of CPR and NADPH, demonstrating that it retained enzymatic activity after immobilization. Later work by our group showed that this immobilization scheme also allowed electrode-driven mediatorless CYP2C9 mediated metabolism of warfarin 
to 7-hydroxywarfarin ${ }^{106}$. This study showed an anaerobic CV scan of the Au/OT-MUA/2C9 electrode revealed quasi-reversible reduction and oxidation peaks at a formal potential of -490 $\mathrm{mV}$ and $-420 \mathrm{mV}$ vs. $\mathrm{Ag} / \mathrm{AgCl}$, well in the range of redox and oxidation peaks of $\mathrm{P} 450 \mathrm{~s}^{159}$. Finally, the study demonstrated that this platform could work as a CYP2C9 biosensor as current-concentration data yielded a fit for $\mathrm{K}_{\mathrm{m}}$ of warfarin hydroxylation $(3 \mu \mathrm{M})$ that is in good agreement with previous literature values for this reaction ${ }^{160}$. More recently, Fantuzzi et al. ${ }^{161}$ have shown rapid determination of $\mathrm{K}_{\mathrm{m}}$ for a set of known substrates for CYP3A4, CYP2C9, and CYP2D6 using a SAM linked P450-electrode integrated into a microtiter plate format.

To gain a complete understanding of protein structure and function, and allow exploitation of their unique properties for bioelectronics, the ability to study single proteins becomes a necessity. The previously mentioned studies all measure a large ensemble of proteins in various conformations, and thus the data represents a statistical average that may occlude specific features. This is especially important for P450s which are prone to catalytic changes due to aggregation. To this end there have been several studies investigating methods to study single proteins. The easiest method for single protein study is the use of electrochemical scanning tunneling microscopy (EC-STM), which has been used to investigate electron transport in isolated azurin ${ }^{162-164}$. This method is ideal because the high resolution of EC-STM allows visualization of single proteins to be measured. However, this method only allows confirmation of isolation, but does not provide a means of controlling aggregation. Secondly, this method does not allow multiple studies to be conducted on the same protein, which prevents study of how characteristics are altered under different conditions.

Another nanoscale method to study single proteins involves creation of nano-gap electrodes through mechanical breaking ${ }^{165,166}$ or electromigration techniques ${ }^{167}$. Our lab has used electromigration to create a nanogap electrodes with $5 \mathrm{~nm}$ gaps between platinum source and drain electrodes deposited on silicon ${ }^{168}$. This narrow gap allowed isolation and interrogation of molecular energy levels of a single molecule of apomyoglobin or myoglobin. The work 
demonstrates that electrons tunnel through the protein, and that the heme group mediates the electron tunneling. The success of this study on heme containing myoglobin is proof that this technique could be possible with P450s. However, this technique has several drawbacks that prevent it from allowing full interrogation of proteins. Creation of working junctions is inefficient and if not performed correctly can result in characteristics of the junction itself being probed, for example when metal grains are formed, rather than the molecule of interest ${ }^{168,169}$. In addition, this technique allows no control over the orientation and conformation of the protein on the electrodes.

As the above methodologies alone do not provide an effective way of isolating unmodified protein, labs have sought to use a multifaceted approach by combining techniques. Work by Smaali et al. ${ }^{170}$ demonstrated the creation of an array of gold nanopillars $8 \mathrm{~nm}$ in diameter using electron beam lithography, which could be functionalized with SAM. Utilizing a similar approach, our lab created an indexed array of gold nanopillars that CYP2C9 could be linked to using a SAM comprised of 3:1 OT:MUA ${ }^{46}$. The indexing of the array allowed multiple studies on the same P450 enzyme exposed to different substrates. The ability to complete repeated studies on the same enzyme demonstrated that ET correlated with rates of metabolism, and that competitive inhibitors not only block the active site, but also inhibit transfer of electrons through the protein.

\subsubsection{Characterization of Immobilized P450s}

To fully interpret results obtained from immobilized proteins, it is crucial to have a deep understanding of the protein layer thickness, the density of adsorbed protein, orientation of the protein, secondary and tertiary structure of the adsorbed protein, protein stability, and most importantly the effect of adsorption on the biological function of the protein ${ }^{171}$. As delineated earlier, many studies of immobilized P450s utilize electrochemical techniques, in particular cyclic voltammetry, are used to ensure P450 electroactivity. These studies can also demonstrate the immobilized P450s are capable of substrate turnover when coupled to 
detection techniques including high performance liquid chromatography and liquid chromatography coupled mass spectrometry ${ }^{172}$. Although these methods offer no information on what orientations of immobilized protein exist, the width of voltammogram peaks have been used to analyze the homogeneity of adsorbed protein. For example the wide peaks observed by Shukla et al. ${ }^{141}$ point to heterogeneous P450 orientation, whereas narrow peaks seen by Yang et al. ${ }^{106}$ point to a more homogenous P450 orientation. These techniques however, give little information on structure and conformation of the protein.

Generally, information about protein secondary and tertiary structure can be obtained by Circular Dichroism (CD) spectroscopy ${ }^{72}$. CD measurements are generally conducted in solution, thus labs have employed the use of nanoparticles to observe how protein adsorption affects structure ${ }^{173,174}$. P450 attributes including heme spin state and binding of substrates can be obtained easily with UV-Vis spectroscopy in solution ${ }^{17,175}$. However, UV-vis spectroscopy can only be conducted on adsorbed proteins if they can be immobilized to quartz ${ }^{176}$. Thus information obtained from the protein immobilized to quartz may not hold true for different solid platforms. To overcome this limitation, other optical techniques can be employed to determine P450 surface coverage and interactions.

Ellipsometry is a spectroscopic technique that allows the determination of layer thickness. By measuring the constructive and destructive interference of light reflecting off the surface or traveling through the film, we can obtain the thickness of the film. Ellipsometry is typically for films with thickness in the range of nanometers to microns. This technique is useful because it can be tailored to study parameters by varying the spectral region of light, which allows protein layer thickness determination on the desired solid platform. An example of this is the use of ellipsometry to measure increases in CYP11A1 layer thickness on a silicon surface after multiple P450 layer applications ${ }^{176}$. In contrast to optical methods, quartz crystal microbalance (QCM) also allows layer thickness determination by measuring changes in oscillating frequency of the substrate upon mass load ${ }^{171}$. Solid support choice in QCM is flexible since the electrodes 
embedding the piezoelectric crystal can be coated with practically any desired thin film.

A very useful optical technique is Surface Plasmon Resonance (SPR), which allows label free study of biomolecular interactions in real time ${ }^{177,178}$. In short, P polarized light reflects off of the backside of a sensor chip, generally glass with a thin film $(\sim 50 \mathrm{~nm})$ of metal deposited on top (Figure 1.8). At a specific incidence angle, known as the resonance angle, the light is absorbed by the electrons in the metal causing them to resonate, and producing a sharp shadow, known as the SPR dip, in the reflected intensity. These resonating electrons are known as surface plasmons, and they are highly sensitive to their surrounding environment. Changes in mass on the surface will cause changes in the resonance angle, and the shape and location of the SPR dip, which can be directly correlated to binding.

By conducting SPR analysis with various concentrations of analytes, flowed over an immobilized protein, a binding dissociation constant $K_{D}$, the ratio of the association "on" rate $\left(K_{a}\right)$ and dissociation "off" rate $\left(K_{d}\right)$, can be obtained. It is important to note that the $K_{D}$ is not equivalent to $K_{m}$, as the $K_{D}$ does not take into account the rate of formation and release of product $\left(K_{2}\right)$. There are also many well studied binding models that data can be applied to interpret observed biomolecular interactions. Studies by Ivanov et al. ${ }^{179}$ demonstrated immobilization of yeast CPR (YCPR) onto functionalized nickel or gold, and evaluated binding of FMN and FAD. The study demonstrates specific binding of FAD and FMN to YCPR, and also concluded a 1:1 binding ratio based on the maximum binding at saturating analyte concentrations. In addition, information about the amount of functionally attached $ү C P R$ can be inferred from the max signal at saturating analyte conditions. This same methodology could be applied to the study of immobilized P450s as evidenced by work on P450cam binding with putidaredoxin ${ }^{180}$. This would thus allow confirmation that immobilized P450 still retain substrate binding activity, and could be used to measure protein-protein interactions. 


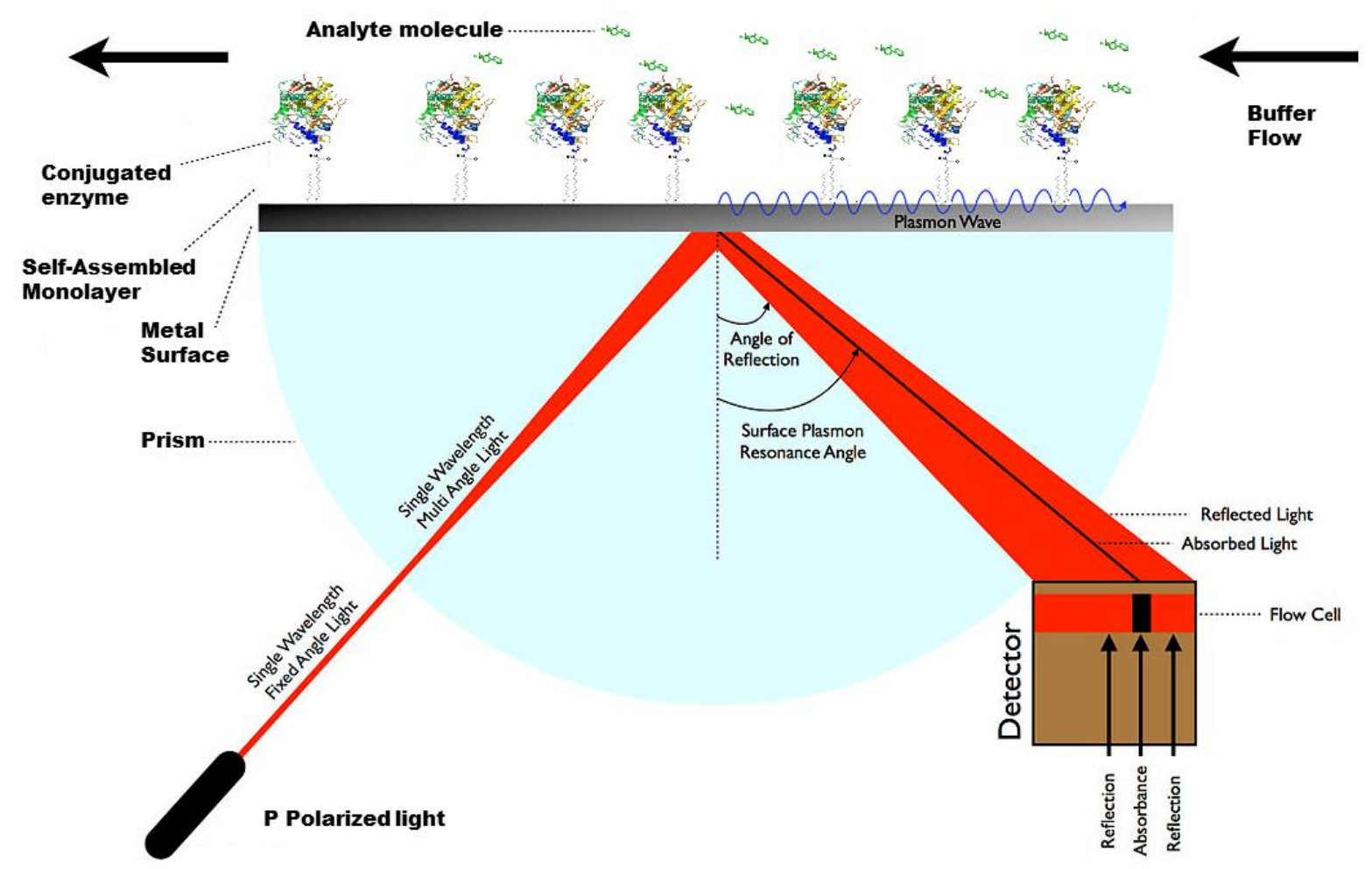

Figure 1.8. Diagram of surface plasmon resonance spectroscopy with a conjugated enzyme system. The black bar in the detector represents the resonance angle where the light is absorbed by the electrons in the metal creating the SPR dip.

Another technique that has been used to measure P450 binding activity and the heme state is surface-enhanced resonance raman spectroscopy (SERRS). Raman spectroscopy relies on inelastic scattering of laser light upon interaction with a sample ${ }^{181}$. Photons are absorbed by the surface and re-emitted. The frequency of the re-emitted photons undergoes a shift based on the vibrational, rotational, and other low frequency transitions in molecules, giving information about the surface. SERRS studies conducted on P450cam immobilized to an MUA SAM on Ag electrodes showed that both the ferric and ferrous states of the enzyme were in the inactive P420 form ${ }^{182}$. A more recent study demonstrated that CYP2D6 immobilized to an MUA SAM on $\mathrm{Ag}$ electrodes was able to reversibly bind its substrate dextromethorphan, and retained its native structure ${ }^{183}$. However, they also observed raman shifts indicative of P420 form, and noted an inability to reduce CYP2D6. This result is interesting given work in our lab demonstrating 
metabolically active CYP2D6 adsorbed to MUA SAM on Au, and may reveal surface specific differences on immobilized P450 function.

Lastly, scanning probe techniques have revealed a great deal of information about immobilized proteins due to their high resolutions. Scanning tunneling microscopy (STM) and electron microscopy have lateral resolutions down to the atomic level, allowing for detailed information of adsorbed protein structure. EC-STM is a powerful tool as it akin to combination of conventional STM and cyclic voltammetry ${ }^{162}$. The conductive substrate and STM tip act as working electrodes in an electrolyte solution. There are also reference and counter electrodes on opposite sides of the tip. The reference electrode is a material, often silver wire, whose potential can be measured to obtain an absolute measurement. The counter electrode allows the current to flow. A bipotentiostat allows independent control of the potential of the substrate and tip with respect to the reference, and their difference represents the tunneling bias. A bias voltage or nuclear configuration fluctuations can cause the energy level of the molecule to be brought closer to the Fermi level of the electrodes, allowing an ET sequence to occur.

In EC-STM electrons tunnel between the protein and the electrodes due to alignment of molecular orbital energy levels of the protein with the Fermi level of the contact electrodes. This alignment with the Fermi levels can be obtained through application of a bias voltage or nuclear fluctuations of the protein ${ }^{164,184}$. Utilizing EC-STM, mechanistic data about ET in proteins and changes in reduction and conformation can be obtained. Thus with P450s we could conduct these studies with different substrates to see what changes in redox state and local confirmation of the heme group occur during the process of electron transfer. We could also monitor changes in the barrier height of P450 in relation to redox state, as has been done in other studies with Azurin ${ }^{163}$. There is currently a lack of studies utilizing EC-STM to investigate immobilized P450. However, work by Hill and co-workers using STM to measure P450cam and its binding to putidaredoxin reveal the possibility of P450 EC-STM studies ${ }^{185-187}$. 
Atomic Force Microscopy (AFM) is another scanning probe technique that is extensively used in protein surface characterization ${ }^{188}$. AFM measures the topography of a surface using a tip consisting of a small probe attached to a cantilever (Figure 1.9). Depending on the AFM

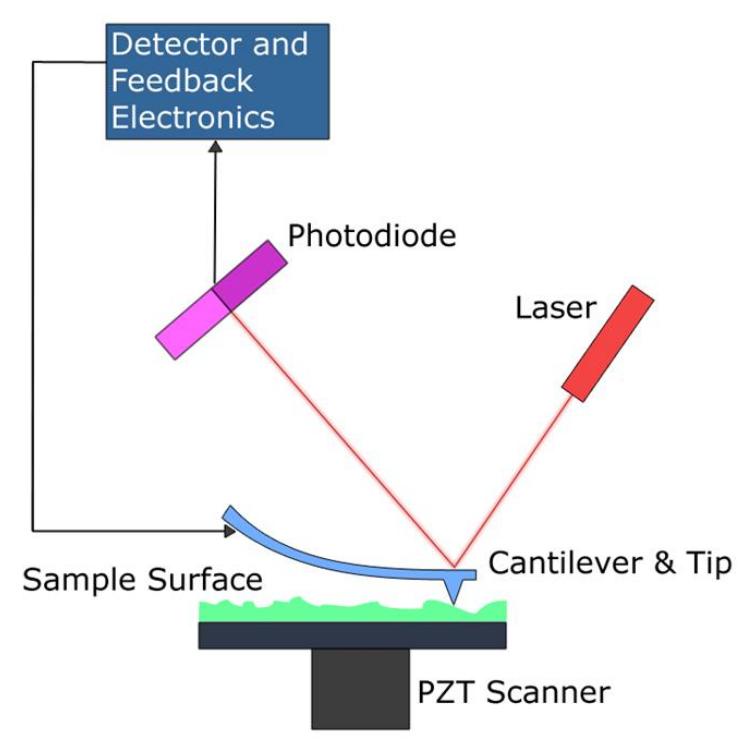

Figure 1.9. Schematic depiction of atomic force microscopy. method, the tip either makes direct contact with the surface (contact mode), or oscillates being driven at its resonance frequency close enough to the surface that van der Waals forces cause changes in its oscillation (tapping mode). This is translated into an image by a laser reflecting off the back of the tip to a photodiode detector and can be visualized in $x, y$, and $z$ axis. With P450s, AFM has

been used to monitor CYP2C9 interaction with a lipid bilayer ${ }^{189}$, determine P450 surface coverage $^{158,189}$, measure P450 isolation by lipid bilayer disc ${ }^{142}$, and demonstrate P450-P450 interactions ${ }^{190}$. Furthermore, a special attachment allows conductive probe atomic force microscopy (CPAFM) in contact mode imaging. In CPAFM, a DC bias differential is applied between the tip and the surface, allowing the system to measure the resistance and conductance of the sample at the site of tip sample interaction. Our lab has used this technique to investigate conductive characteristic of single CYP2C9 molecules immobilized to gold nanopillars after exposure to different substrates ${ }^{46}$. In addition, the work in chapter 3 demonstrates the unique ability of AFM to measure stability of P450 structure, and the use of height analysis to confirm isolation.

\subsection{Project Goals}

The accuracy of in vitro assays in predicting in vivo drug metabolism is of crucial importance to avoid interactions that cause non-therapeutic doses of pharmaceuticals. Currently, we use 
reconstituted systems that contain the full complement of necessary proteins and co-factors in solution to assess kinetic parameters. However, it is well known that the free floating P450 nature in these systems does not match the membrane bound environment found in vivo. It has also been demonstrated that P450s can aggregate into homo/heteromeric complexes in solution, which have been shown to alter their catalytic rates. To further complicate things, we know that the levels of necessary cofactor CPR are drastically lower than the amount of P450s, and our understanding of how electrons are transferred to each P450 is incomplete.

To address these issues it is necessary to develop methodologies that allow studies of nonaggregated P450s. Previous attempts to disaggregate P450s using detergents and ionic buffers have resulted in changes of P450 catalytic activity due to the disaggregating agent. To improve upon this we need to be able to isolate a P450 of interest in an enzymatically unchanged manner. Achieving this will allow us to understand inherent properties of metabolism, and how they are altered in the presence of effectors. This lends itself to the study of substrate effectors as well as P450-P450 interactions. To accomplish this an interdisciplinary approach must be taken as a means to control the P450 aggregation state without significant alteration of its native state.

Our long-term goal is to develop methods to gain a better understanding of P450 function, and to improve our design and interpretation of in vitro P450 metabolism towards better prediction of in vivo P450 metabolism. We intended to accomplish this through the use of P450 immobilization to control aggregation, yet preserve P450 functionality. This allows for careful study of an enzymatically active P450 without the variable of aggregation. In addition, the ability to immobilize the P450 grants greater control allowing single molecule studies under different and defined conditions.

Project Aims:

1) To use an immobilized P450 platform to control aggregation, and allow quantification of different P450-P450 interactions and their resulting metabolic effect. 
2) Create a nanoscale platform for isolation of a single P450, and study its conductive properties in the presence of various substrates to gain insight on the critical electron transfer process.

This dissertation will discuss the use of an immobilized platform designed to control P450 aggregation. The immobilized platform consisting of 3:1 MUA:OT on gold chips was used to immobilize CYP2C9. Using this SPR experiments were then conducted to measure homo/heteromeric complex formation of P450s in real-time, and obtain kinetic binding parameters. The effects of P450 complex formation on CYP2C9 mediated metabolism was evaluated using the same immobilized platform as SPR experiments. To gain a greater mechanistic understanding of the effects, data was evaluated in conjunction with molecular modeling data of $\mathrm{P} 450$ binding pairs and regions of interest that might be affected.

Furthermore, I will discuss the use of a nanoplatform using the same immobilization scheme as above to further control P450 aggregation. Using e-beam lithography a chip with isolated gold nanopillars of controlled size was created to immobilize single CYP2C9 molecules. This array is indexed to allow for repeated studies of the same molecule under varying conditions. I demonstrated the use of a CYP2C9-coated nanopillar array platform for the purpose of measuring ETp in the absence or presence of various substrates or inhibitors molecules of CYP2C9. Ultimately, the development of this platform will allow us to better understand the process of electron transfer in P450 enzymes which can enhance our mechanistic understanding of P450 metabolism, and allow for better drug design and metabolism predictions. 


\section{Chapter 2}

\section{Immobilized Cytochrome P450 for Monitoring of P450-P450 Interactions and Metabolism}

Adapted from Bostick C.D*., Hickey, K.M*., Wollenberg L, Flora, D.R., Tracy T.S., \& Gannett PM. Immobilized Cytochrome P450 for monitoring of P450-P450 Interactions and Metabolism. Pending Revision.

* Denotes co-first author 


\subsection{Introduction}

Cytochrome P450s (P450s) are a superfamily of monooxygenases responsible for metabolizing $75 \%$ of the pharmaceuticals on the market as well as other xenobiotics and endobiotics ${ }^{13}$. They are found primarily in the liver where they are membrane bound via their hydrophobic $\mathrm{N}$-terminus to the smooth endoplasmic reticulum ${ }^{11}$. Their mechanism of action is dependent upon the sequential transfer of two electrons. The first transfer requires cytochrome P450 reductase (CPR), and the second transfer requires CPR or CPR and cytochrome $b_{5}(\mathrm{~b} 5)$ 16. While CPR is required for P450 metabolism, b5 is not required for P450-mediated metabolism, and its presence, in certain instances, simply enhances metabolism ${ }^{17-19}$. In microsomes, P450 levels exceed those of CPR by at least five fold ${ }^{25,26}$ suggesting enzyme mobility may be required ${ }^{27,28}$, or multiple P450s utilize a single CPR for the supply of electrons from NADPH ${ }^{29-31}$.

The effect of P450-P450 and P450-CPR interactions on liver metabolism is poorly characterized. It has been suggested that P450s are clustered rather than being uniformly distributed in the endoplasmic reticulum ${ }^{191}$ and that there may exist zonal differences ${ }^{192}$. Under in vitro conditions it has been shown that $\mathrm{P} 450$ s interact ${ }^{67,68}$, and that this interaction modulates P450-mediated metabolism ${ }^{81,193}$. This effect on metabolism has been seen in multiple reconstituted enzyme systems including human CYP2C9-CYP2D6 ${ }^{194}$, human CYP2C9CYP3A4 ${ }^{78}$, human CYP2C9-CYP2C19 ${ }^{64}$, rabbit CYP1A2-CYP2B4 ${ }^{66,195}$, rabbit CYP1A2CYP2E1 ${ }^{74}$, and human CYP3A4-CYP1A1 and CYP3A4-CYP1A2 ${ }^{18}$. P450-P450 interactions

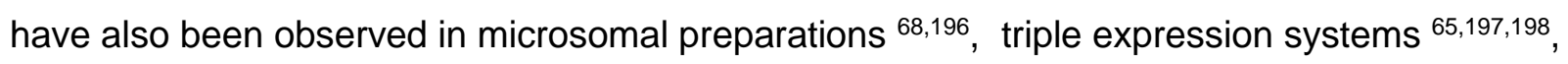
and homomeric oligomerization of CYP2E1 ${ }^{199}$ CYP2B4 ${ }^{200}$ and CYP3A4 ${ }^{143,201}$.

While P450-P450 interactions in vitro have been found to affect metabolism, there remains some ambiguity as some studies have reported that P450-P450 interactions did not affect metabolism. Dutton et al. (1987) reported no effect on P450-mediated testosterone metabolism 
in the presence of other P450s in rat microsomes ${ }^{202}$. Alston et al. (1991) showed the formation of heterodimers by some P450s but not others suggesting there is some selectivity for the formation of heteromeric P450 complexes. Elucidating the underlying factors controlling P450P450 interactions is essential for accurately predicting in vivo P450 substrate clearance rates from in vitro data. For example, predictions of clearance are often based on observations in single isoform reconstituted systems that, therefore, lack the possibility of observing effects due to hetero-aggregate formation. Also, while the aforementioned studies have shown oligomerization does occur in solution, the extent and characterization of how P450s interact in vivo has yet to be examined.

There are three main mechanisms by which one P450 can affect the metabolic rate of another: competition for CPR binding, the formation of a complex with altered affinity for CPR, and the formation of a complex with altered substrate binding or turnover rate ${ }^{81,193}$. Recently there has been interest in the use of controlled immobilization of P450s to allow better understanding of metabolism ${ }^{143,149,155,158,161,203,204}$, and the formation of homo/heteromeric complexes ${ }^{193}$. Our lab has developed a scheme whereby soluble P450 is covalently attached to a self-assembled monolayer (SAM) on a gold film. The result retains full metabolic activity and uses the normal protein binding partners and cofactors to metabolize substrates ${ }^{158}$. This platform offers a model system for how P450 is bound in vivo and thus allows for control of P450 aggregation. In the present work we assess the interaction of CYP2C9 with three major P450 isoforms (CYP2C9, CYP3A4, and CYP2D6) and with CPR, demonstrate the formation of homo/heteromeric P450 complexes in real-time, and determine the consequences of these complexes on P450-mediated metabolism. The results are also supported by molecular modeling studies of the P450 complexes.

\subsection{Materials and Methods}

\subsubsection{Chemicals and Reagents}


All chemicals were used as purchased. Potassium phosphate, HEPES, sodium chloride, 11-mercaptoundecanoic acid (MUA), 1-octanethiol (OT), N-(3-dimethylaminopropyl)-N'ethylcarbodiimide hydrochloride (EDC), N-hydroxysulfosuccinimide sodium salt (NHS), flurbiprofen, dilauroylphosphatidylcholine (DLPC), and beta-nicotinamide adenine dinucleotide phosphate reduced form (NADPH), Cetyltrimethylammonium bromide (CTAB), were purchased from Sigma-Aldrich (St. Louis, MO). CYP2C9 was prepared by expression in an Escherichia coli system, isolated, and purified as described previously ${ }^{17,205}$. Human CPR was purchased from Invitrogen (Carlsbad, CA). All solutions were prepared in $18 \mathrm{M} \Omega-\mathrm{cm}$ water unless otherwise noted. Phosphate buffer (40 mM, pH 7.4) contained $150 \mathrm{mM}$ sodium chloride (PBS). HEPES buffer $(10 \mathrm{mM}, \mathrm{pH}=7.4)$ contained $150 \mathrm{mM}$ sodium chloride, $3 \mathrm{mM}$ EDTA, and $0.005 \% \mathrm{v} / \mathrm{v}$ Surfactant P20. Titanium and gold pellets were purchased from Kurt J. Lesker Company (Jefferson Hills, PA). Cryobuffer was made from PBS and glycerol (80:20 v/v).

\subsubsection{Molecular Modeling}

Computational modeling was employed to determine possible enzyme binding orientations and the relative binding energy of the enzyme pairs CYP2C9-CYP2C9, CYP2C9-CYP3A4, CYP2C9-CYP2D6, and CYP2C9-CPR. To account for conformational changes affecting the binding strength and binding site, several CYP2C9 crystal structures were examined including 1OG2 (without substrate), 1OG5 (with bound warfarin, closed) ${ }^{206}$, and $1 \mathrm{R} 90$ (with bound flurbiprofen, partially open) ${ }^{207}$. Structural similarity was assessed using the MultiSeq module 208 of VMD. P450 structures, crystallized without substrate, were used to match the experimental conditions: CYP2D6 (Rowland et al., 2006, PDB ID 2F9Q) and CYP3A4 (Yano et al., 2004, PDB ID 1TQN). The crystal structure selected for CPR is locked into a conformation adopted when binding to a P450 enzyme due to a TGEE truncation in the hinge region (Hamdane et al., 2009, PDB ID 3ES9 chain A). GRAMM-X ${ }^{212}$ was used to computationally predict binding modes and 
the ten lowest potential energy models per enzyme pair were selected for analysis. All homoand heterodimers were examined in VMD ${ }^{213}$ with respect to residues defining areas of interest as reported in the literature including the active site and solvent tunnel entrances ${ }^{214}$, the CPR binding site $211,215,216$, and the truncated $\mathrm{N}$-terminus. The relative binding energy for the lowest energy GRAMM-X models for each binding pair was calculated using MM GBSA ${ }^{217}$ after minimization, equilibration and $2 \mathrm{~ns}$ molecular dynamics at $300 \mathrm{~K}$ using the Amber software suite ${ }^{218-220}$.

\subsubsection{Gold-OT/MUA-CYP2C9 Chip Fabrication}

Two different materials were used as test platforms for the experiments described: gold coated silicon wafers for generation of metabolites, and gold coated glass cover slips for SPR detection of binding. Both were prepared for surface modification using a Temescal BJD-2000 (Edwards Vacuum, Phoenix, AZ) system with an Inficon XTC/2 deposition controller (Inficon, East Syracuse, NY). Evaporation occurred at a voltage of $10.0 \mathrm{kV}$. The current was set so that metal vaporization occurred at a rate of $0.03-0.10 \mathrm{nM} / \mathrm{s}$ : approximately $50 \mathrm{~mA}$ for titanium and $100 \mathrm{~mA}$ for gold. Thin films comprised of titanium, $(\sim 5 \mathrm{~nm})$ thick, and then gold $(\sim 50 \mathrm{~nm})$ thick, were deposited on silicon chips or glass cover slips. Silicon wafer platforms were diced into 4 $\mathrm{mm} \times 6 \mathrm{~mm}$ chips and then were sonicated in a Bransonic 220 (Bransonic, Danbury, CT) (5 $\min$ ) and rinsed in succession with acetone, isopropyl alcohol, and water (each, 3X) to ensure a clean surface. Glass platforms were cut into $10 \mathrm{~mm}$ x $12 \mathrm{~mm}$ rectangles, sonicated (2 min), and rinsed with isopropyl alcohol and then water (each, 3X). A self-assembled monolayer (SAM) was then formed on the gold surface by first rinsing it with ethanol followed by soaking it in an ethanolic solution of OT (7.5 mM) and MUA (2.5 mM), overnight under argon. Excess thiol was removed by rinsing, in succession, with absolute ethanol, 95\% ethanol, and water (each, 3X). Chips were dried under a gentle stream of nitrogen. 


\subsubsection{Surface Plasmon Resosnance Binding Analysis}

Glass platforms coated with gold-SAM as described above were loaded into a Biacore X100 SPR (GE Healthcare, Pitscataway, NJ) for further surface modification and analysis. The surface was then activated with $\operatorname{EDC}(0.4 \mathrm{M})$ and $\mathrm{NHS}(0.1 \mathrm{M})$ in PBS running buffer as per Biacore instructions. CYP2C9 (100 nM) in PBS running buffer was flowed (10 $\mu \mathrm{L} / \mathrm{min})$ over the test surface (480 s), capped with ethanolamine (1 M) in PBS running buffer, and then rinsed with PBS running buffer alone (480 s). The control surface was prepared by directly capping it with ethanolamine. Immobilization levels reaching 70 to 200 response units above the control surface, post running buffer rinse, were considered acceptable. Analysis of binding between immobilized and soluble enzyme (0, 5, 10, 50, 100, $500 \mathrm{nM})$ was conducted in PBS running buffer at $30 \mu \mathrm{L} / \mathrm{min}$. After stabilization, contact times for soluble enzyme were $180 \mathrm{~s}$ followed by 900 s dissociation. Flow cells were regenerated with CTAB $(3.9 \mathrm{mM})$. Two concentrations of the soluble enzyme $(50,100 \mathrm{nM})$ were measured twice to ensure reproducibility of data. Each P450 binding pair was run at least three times. Fitting of kinetic data was accomplished using both Biacore x100 Evaluation software and Scrubber 2.0 (BioLogic Software, Campbell, Australia). CYP2C9, CYP3A4, and CPR were fit using Biacore x100 Evaluation software. Biacore x100 Evaluation was unable to fit CYP2D6 curves, and Scrubber 2.0 was employed instead. Cross analysis between fitting software's demonstrated consistency.

\subsubsection{Immobilization of CYP2C9 Enzyme on Gold/Silicon Platform}

To bond the CYP2C9 to the SAM, the SAM-coated slides were first activated by immersion in an aqueous solution of EDC $(2 \mathrm{mM})$ and $\mathrm{NHS}(5 \mathrm{mM})(1: 1 \mathrm{v} / \mathrm{v})$, each in water, for $2 \mathrm{~h}$ at room temperature (RT). Activated chips were rinsed with PBS, then soaked (24 h, RT) in a solution containing CYP2C9 (100 nM), flurbiprofen $(40 \mu \mathrm{M})$, and dapsone $(40 \mu \mathrm{M})$ in PBS, under argon, 
to covalently bond CYP2C9 to the NHS-esters. We have shown inclusion of substrates flurbiprofen and dapsone preserves CYP2C9's active site integrity, and allows retention of enzymatic activity through immobilization ${ }^{158}$. Chips bearing the bonded enzyme were rinsed with PBS to remove substrates from the active site before placing in cryobuffer solution and storing at $-80^{\circ} \mathrm{C}$ for at least $8 \mathrm{~h}$ prior to use.

\subsubsection{Reconstituted CYP2C9 Enzyme Incubation}

Reconstituted CYP2C9 incubations were run in triplicate. Incubations contained CYP2C9 (0.25 nM), saturating CPR (1-4 nM), DLPC (10 $\mu \mathrm{g})$, and flurbiprofen $(160 \mu \mathrm{M})$ in PBS (40 mM, $\mathrm{pH}$ 7.4) for a total volume of $250 \mu \mathrm{L}$. Immobilized CYP2C9 (CYP2C9i) incubations were conducted using two $4 \mathrm{~mm} \times 6 \mathrm{~mm}$ gold chips placed back to back (gold coating facing outwards). DLPC was omitted in incubations containing CYP2C9 immobilized to the gold substrate. Coverage of CYP2C9 on gold chips was estimated based on previous work ${ }^{106,158 .}$ Ratios of CYP2C9i to CYP2C9 in solution (CYP2C9s) ranged from 1:1 to 1:4. Ratios of CYP2C9/CYP2D6 and CYP2C9/CYP3A4 ranged from 1:0.25 to $1: 8$ and $1: 1$ to $1: 12$, respectively, to mimic possible in vivo ratios of these enzymes ${ }^{221}$. Incubations were placed on ice during the addition of pre-cooled solutions of reactants and then preheated ( $3 \mathrm{~min})$ at $37^{\circ} \mathrm{C}$ before the initiation the incubation by the addition of NADPH ( $200 \mu \mathrm{M}$, final concentration) and then incubated at $37^{\circ} \mathrm{C}$ overnight ( 16 hours). Reactions were terminated by the addition of 10 $\mu \mathrm{L}$ of $500 \mathrm{ng} / \mathrm{mL}$ 2-Fluoro-4-biphenyl acetic acid (Internal Standard) and $30 \mu \mathrm{L} 50 \%$ phosphoric acid. Incubation mixtures were centrifuged (10 min, 13,400 x g) to pellet proteins, and supernatant $(135 \mu \mathrm{l})$ loaded into LC vials for analysis.

For incubations containing heterodimers, the effect of the order of mixing of P450s and CPR was investigated by i) pre-incubating CYP2C9 (4 nM) with CPR (5 min) prior to the addition of CYP3A4 or CYP2D6 or ii) pre-incubating CYP2C9 (4 nM) with CYP3A4 or CYP2D6 (5 min) prior 
to addition of CPR. In studies investigating the order of addition, CPR was sub-saturating with a ratio less than 1 P450:2 CPR. Incubations were placed on ice during the addition of pre-cooled solutions of reactants and then preheated $(3 \mathrm{~min})$ at $37^{\circ} \mathrm{C}$ before initiation of the incubation by the addition of NADPH $\left(200 \mu \mathrm{M}\right.$, final concentration) and then incubated at $37^{\circ} \mathrm{C}$ for the indicated time. Reactions were terminated by the addition of $10 \mu \mathrm{L}$ of $500 \mathrm{ng} / \mathrm{mL} 2$-fluoro-4biphenyl acetic acid (internal standard) and $30 \mu \mathrm{L} 50 \%$ phosphoric acid. Incubation mixtures were centrifuged (10 min, 13,400 x g) to pellet proteins, and supernatant (135 $\mu \mathrm{L}$ ) loaded into LC vials for analysis. All experiments were performed three times on separate days.

\subsubsection{HPLC Assay of 4'-Hydroxyflurbiprofen}

Metabolite formation, 4'-hydroxyflurbiprofen, was measured by HPLC with fluorescence detection. The HPLC system consisted of an Alliance 2695XE pump/autosampler and a 2495 fluorescence detector (Waters, Milford, MA) set at an excitation wavelength of $280 \mathrm{~nm}$ and an emission wavelength of $310 \mathrm{~nm}$. The mobile phase consisted of potassium phosphate $(20 \mathrm{mM}$, $\mathrm{pH} 7.4)$ and acetonitrile $(50: 50 \mathrm{v} / \mathrm{v}) ; 0.75 \mathrm{~mL} / \mathrm{min}$ flow-rate; 4.6 x $150 \mathrm{~mm}$ Zorbax $\mathrm{C}_{18}$ column (Agilent, Santa Clara, CA). The retention times of 4'-hydroxyflurbiprofen and the internal standard were 4.1 and 7.7 min, respectively. Metabolite concentration was determined using a calibration curve of 0-50 ng of 4'-hydroxyflurbiprofen.

\subsubsection{Statistical Analysis}

The results for metabolism studies are expressed as the means ( \pm S.E.) and were analyzed by a one-way analysis of variance (ANOVA) followed by Dunnett's test for multiple comparisons with the positive control group. One-way ANOVA with Dunnett's post test was performed using GraphPad Prism version 6 for Windows (GraphPad Software, La Jolla California USA, www.graphpad.com).

\subsection{Results}




\subsubsection{Computationally Predicted Dimers}

P450s are known to form dimers and multimers and their formation is believed to impact metabolic activity. Here, modeling approaches were used to predict the likely binding poses of homo- and heterodimers, the consequence of dimer formation on metabolic activity, and the stability of the dimers relative to the monomers. The enzyme pairs examined were CYP2C9CYP2C9, CYP2C9-CYP3A4, CYP2C9-CYP2D6, or CYP2C9-CPR. For each pair only the CYP2C9 residues involved in binding were considered and, in particular those, involved in CPR binding 211,215 , the solvent access channel, and the substrate channel entrance ${ }^{214,222}$ (Table 2.1).

Table 2.1 CYP2C9 regions of interest and the relevant residues.

\begin{tabular}{l|l}
\hline \multicolumn{1}{c|}{ Region of Interest } & \multicolumn{1}{c}{ CYP2C9 Residues } \\
\hline Substrate Access Channel & P37 I42 I45 I47 F69 K72 I74 R97 G98 I99 P101 L102 A106 R108 G109 \\
& P211 N218 P221 I222 H230 V237 288 \\
Solvent Channel & C206 E300 R307 F476 \\
CPR Binding Site & K84 D89 E92 K121 R125 R132 K270 K273 K421 K423 K432 E438 \\
P450 Binding Site on CPR & D208 \\
a223 & \\
b216 &
\end{tabular}

It has been demonstrated that P450s exist in both open and closed conformations ${ }^{224}$. The closed conformation is favored upon substrate binding $206,210,225$ and alters the affinity toward enzyme partners ${ }^{216,226}$. To account for potential conformational changes on the modeling results, dimer formation was examined with an open and closed form of CYP2C9. To select these structures, three CYP2C9 crystal structures were considered: 1OG2 (without substrate), 1OG5 (with bound warfarin) ${ }^{206}$, and 1R9O (with bound flurbiprofen) ${ }^{207}$. The 1OG2 and 10G5 models, that represent open and closed states, respectively, were more structurally similar to each other than to $1 \mathrm{R} 9 \mathrm{O}$, a partially open conformation. Therefore, further analysis was conducted only on the 1OG5 (closed) and 1R9O (partially open) models. 
For the purpose of generating dimer structures, blind docking, using GRAMMX, was performed for each dimer combination. Each of the ten models returned by GRAMMX for the homo- and heterodimers were examined and divided into three groups, a) those with at least $40 \%$ of the identified residues in the interface of the dimer, b) less than $40 \%$ overlap, and c) where dimer formation occurred but remote from the region of interest ${ }^{211}$. The occurrence of each interaction type and frequency is shown in Table 2.2.

Table 2.2 Dimer Binding Sites Predicted by GRAMMX.

\begin{tabular}{l|llll}
\hline \multirow{2}{*}{ CYP2C9 Model } & Binding Partner & $\begin{array}{l}\text { Active Site Access } \\
\text { Channel }^{\mathrm{b}}\end{array}$ & $\begin{array}{l}\text { Solvent Access } \\
\text { Channel(s) }^{\mathrm{b}}\end{array}$ & CPR Binding Site $^{\mathrm{b}}$ \\
\hline \multirow{4}{*}{1 R9O } & CYP2C9c & 1 & 2 & 2 \\
& CYP3A4 & 2 & 2 & 1 \\
& CYP2D6 & 2 & 2 & 1 \\
& CPR & 3 & 4 & 0 \\
\multirow{4}{*}{$10 G 5$} & CYP2C9c & 2 & 6 & 0 \\
& CYP3A4 & 0 & 5 & 1 \\
& CYP2D6 & 0 & 3 & 1 \\
& CPR & 1 & 6 & 0 \\
\hline aEch dimer contained either 1R90 or 10G5 and the indicated binding partner. The structure of the binding partner was obtained
\end{tabular}
from 19RO.pdb for CYP2C9, 1TQN.pdb for CYP3A4, 3TBG for CYP2D6, or 3ES9.pdb for CPR. ${ }^{\circ}$ Numbers indicate number of models out of the top ten models displaying interaction in specified region. Interactions for each of the top ten binding models was considered. Dimerization involved the solvent or substrate channels $25 \%$ of the time and the CPR binding site about $7.5 \%$ in predicted models.

Dimerization most frequently involved the solvent access channel region of CYP2C9 (Table 2.2). In the case of the closed CYP2C9 (1OG5) this location was heavily favored. In the case of the partially open model, the solvent access channel region was still the favored site with the solvent access channel only slightly less preferred. In either case, the CPR binding site was the least preferred site. The distinctions between the three sites were greatest for the closed model. (Figure 2.1). 
a)

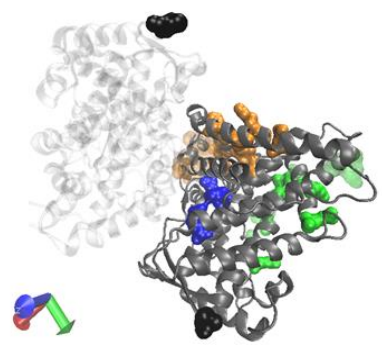

c)

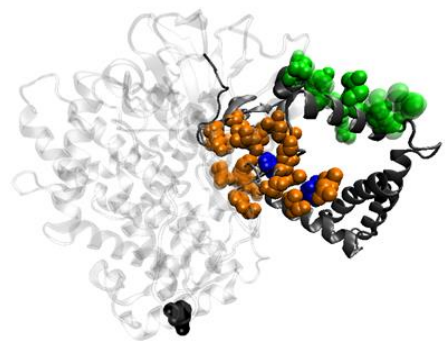

b)

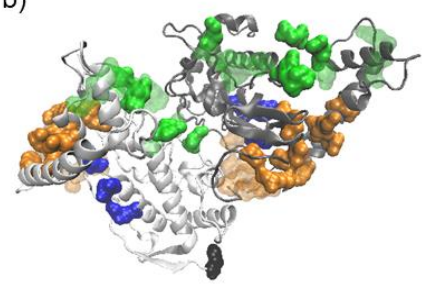

d)

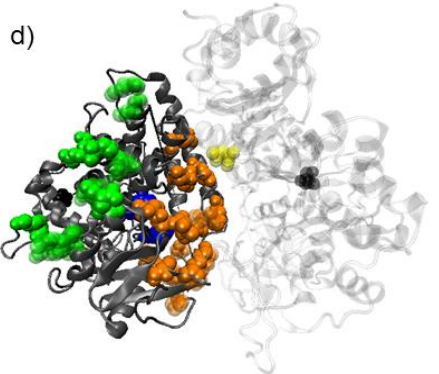

Figure 2.1. Representation of the top scored interaction predicted by GRAMMX for $1 R 90$ bound to CYP3A4, CYP2C9, CYP2D6, and CPR. Residues defining the CPR binding site (green), substrate access channel entrance (orange), and the solvent channel entrance (blue), are highlighted while other residues have been removed from view. Partner molecules are light gray. a) 1R9OCYP3A4 solvent channel interface area. Binding as depicted by this model could affect substrate turnover rate. b) 1R9O-CYP2C9 CPR binding site interface area. Additionally, the partially blocked surface could affect the strength of the CYP2C9-CPR binding interaction for the CYP2C9 (white) molecule. c) 1R9O-CYP2D6 active site area. Binding as depicted by this model could affect substrate entry and exit into the active site. d) 1R9O-CPR substrate access channel area. Dimerization as depicted in this model could affect substrate recognition, binding, and turnover rate. 
Models were examined with respect to the relative orientation between the CPR binding sites of each monomer (Table 2.3). Previous work has shown that the affinity of CPR for P450s is dependent on P450 dimerization and the relative orientation of the two P450s involved. Therefore, the relative orientation of the binding partner P450 with respect to the CYP2C9 model (1R9O or 1OG5) may affect CPR binding strength and, therefore, the rate of P450 mediated metabolism relative to the interaction between a monomeric P450 and CPR (Table 2.4).

Table 2.3 Residues Involved in CYP2C9 CPR Binding ${ }^{a}$

\begin{tabular}{|l|lllll|lllllll|}
\hline \multicolumn{10}{|c|}{ CPR Binding Site } \\
\hline CYP2C9 & E92 & R125 & R132 & K421 & K432 & K84 & D89 & K121 & K270 & K273 & K423 & E438 \\
CYP3A4 & Y99 & & T138 & & P439 & K91 & K96 & K127 & & & P429 & M445 \\
CYP2D6 & E96 & R133 & R140 & K429 & R440 & R88 & T93 & R129 & E278 & K281 & E431 & E446 \\
\hline
\end{tabular}

${ }^{a}$ Residues involved in CYP2C9 CPR binding were mapped onto CYP3A4 and CYP2D6. Those residues exhibiting strong structural similarity with Qres values greater than 0.75 are reported. CYP2C9 residues listed in the right hand side of the table were reported in the CYP2C9-CPR interface for multiple models, those on the left were reported for a single model.

The orientations that may affect CPR binding strength are shown in Figure 2.2. In this figure the CPR binding site is indicated by the 'notch' in the circle and the latter represents the rest of the P450. The circle on the left is CYP2C9 (1R90 or 1OG5) and that on the right is the partner P450. Basically, the proximity of the CPR binding site on one P450 and the binding site of the partner can affect CPR affinity and the orientations shown in Figure 2.2 will alter CPR affinity. Planar alignment (aa) of both partner CPR binding sites has been correlated with increased CPR binding in comparison to the monomer ${ }^{216}$. In contrast, if the resulting dimer leads to total (ab) or partial (ao) occlusion of the CPR binding site, we expect reduced CPR binding. Lastly, a dimer pair where both CPR binding sites are remote (a-) would be expected to have no effect on CPR binding. 


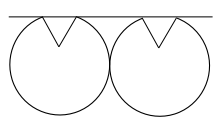

a a

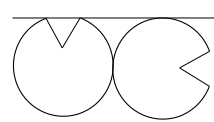

$\mathrm{a}-$

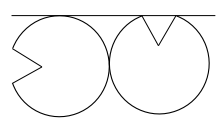

$-\mathrm{a}$

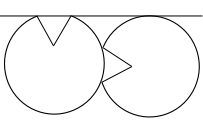

$\mathrm{a} \mathrm{b}$

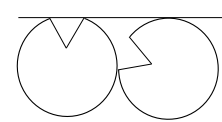

a $\mathrm{O}$

Figure 2.2. Relative arrangements of CYP2C9 (1R9O or 1OG5) and partner P450s (CYP2C9, CYP3A4, and CYP2D6). The left-side corresponds to CYP2C9 (1R9O or 1OG5) and the right-hand side to the partner in each pair. 'Planar alignment' refers to a line that passes over the CYP2C9 CPR binding site and the partner enzyme. The abbreviation 'a' indicates alignment with the partner and implies proximity, ' $b$ ' indicates one of the two CPR binding sites is blocked in the dimer, 'o' indicates partial overlap of one P450-CPR binding site in the complex, and a '-' indicates one of the P450-CPR binding sites is remote from that of its partner.

\begin{tabular}{|c|c|c|c|c|c|c|c|c|c|c|c|}
\hline & & 1 & 2 & 3 & 4 & 5 & 6 & 7 & 8 & 9 & 10 \\
\hline \multirow[t]{3}{*}{$1 \mathrm{R} 90$} & $2 \mathrm{C9}$ & -- & -- & a o & -- & -- & a o & -- & -- & -- & -- \\
\hline & 3A4 & $-a$ & $a b$ & a - & $-a$ & $-a$ & $-a$ & -- & -- & a - & a - \\
\hline & 2D6 & $a-$ & $-b$ & $a b$ & $-a$ & $-a$ & $a-$ & $a b$ & -- & $-a$ & $-b$ \\
\hline \multirow[t]{3}{*}{ 10G5 } & $2 \mathrm{C9}$ & $\mathrm{a} a$ & -- & -- & a - & -- & -- & a - & -- & -- & -- \\
\hline & $3 \mathrm{~A} 4$ & $-b$ & -- & -- & -- & $-a$ & a a & a - & $-a$ & -- & -- \\
\hline & 2D6 & $a b$ & -- & $-a$ & $\mathrm{a} a$ & -- & -- & -- & $-a$ & $-b$ & a - \\
\hline
\end{tabular}

${ }^{a}$ For each model number entry, the first character refers to the CPR binding site of either 1R9O or $10 G 5$ and the second to the partner (CYP2C9, CYP3A4, CYP2D6, CPR). The abbreviations 'a', 'b', and 'o' are explained in Figure 2 and the text.

In approximately $10 \%$ of the models the CYP2C9 CPR binding site was in planar alignment with its enzyme partner, potentially changing CPR binding affinity. Of those, three alignments involved CPR binding regions on both enzyme molecules such as was seen in panel B of Figure 2.1. None of the models resulting from blind docking of CYP2C9 with CPR involved the residues required for a functional CPR-P450 interaction. This result agrees with literature reports that use homology modeling and directed docking to visualize P450-CPR functional dimerization ${ }^{211,216 .}$

\subsubsection{Binding Free Energy in silico}

Computational modeling was employed to determine relative binding energy between four enzyme pairs, CYP2C9-CYP2C9, CYP2C9-CYP3A4, CYP2C9-CYP2D6, and CYP2C9-CPR. Molecular dynamics were run on these structures (neutralized and fully solvated with explicit waters. Binding free energies were calculated using the Molecular Mechanics/Generalized Born Surface Area (MM/GBSA) method, which has been shown to be more successful in ranking binding affinities than the Molecular Mechanics/Poisson Boltzmann Surface Area (MM/PBSA) 
method, though it is less successful in calculating absolute energy values ${ }^{227}$. Resultant energies are reported in $\mathrm{kcal} / \mathrm{mol}$ in Table 2.5. Negative values for total binding free energy suggest favorable protein-protein complexes. The rank-order of the dimers is, from strongest to weakest, CYP2C9-CPR, CYP2C9-CYP2D6, CYP2C9-CYP2C9, and CYP2C9-CYP3A4.

Table 2.5. MM-GBSA Binding Free Energies for CYP2C9 Dimers ${ }^{a}$

\begin{tabular}{|l|r|r|r|r|}
\hline Energy Component & \multicolumn{1}{l|}{ CPR } & \multicolumn{1}{l|}{ CYP2D6 } & \multicolumn{1}{l|}{ CYP2C9 } & \multicolumn{1}{l|}{ CYP3A4 } \\
\hline VDW & $-124.2(6.9)$ & $223.9(9.9)$ & $-157.5(6.4)$ & $-115.7(6.15)$ \\
\hline EEL & $-500.0(43.2)$ & $-88.9(28.3)$ & $-224.3(34.6)$ & $-238.0(35.3)$ \\
\hline EGB & $548.2(38.9)$ & $249.8(28.7)$ & $332.9(33.5)$ & $324.7(32.6)$ \\
\hline ESURF & $-17.23(0.8)$ & $-28.2(1.2)$ & $-20.6(0.7)$ & $-14.5(0.6)$ \\
\hline$\Delta G$ (Gas) & $-624.3(42.0)$ & $-312.7(32.8)$ & $-381.8(34.8)$ & $-353.7(35.3)$ \\
\hline$\Delta G$ (Sol) & $531.0(38.7)$ & $221.6(28.0)$ & $312.4(33.3)$ & $310.2(32.7)$ \\
\hline$\Delta G$ (Total) & $-93.26(6.9)$ & $-91.1(8.2)$ & $-69.4(5.9)$ & $-43.5(6.28)$ \\
\hline
\end{tabular}

Calculated binding free energies for top scored interaction predicted by GRAMMX for 1R90 bound to CYP3A4, CYP2C9, CYP2D6, and CPR. ${ }^{a}$ All values are in $\mathrm{kcal} / \mathrm{mol}$. Values in parenthesis are standard deviations. ${ }^{\mathrm{b}} \mathrm{VDW}=\mathrm{van}$ der Waals contribution from the Molecular Mechanical energy, EEL = electrostatic energy, EGB = the electrostatic contribution to the solvation free energy calculated by the generalized Born approach, ESURF = nonpolar contribution to the solvation free energy calculated by an empirical model, $\Delta \mathrm{G}$ (gas) $=$ VDW $+\mathrm{EEL}, \Delta \mathrm{G}(\mathrm{Sol})=\mathrm{EGB}+\mathrm{ESURF}, \Delta \mathrm{G}$ (total) $=\Delta \mathrm{G}$ (gas) $+\Delta \mathrm{G}($ Sol). $\Delta \mathrm{G}$ (total) binding free energy $[(\Delta \mathrm{G}$ Complex $)-(\Delta \mathrm{G}$ Receptor $+\Delta \mathrm{G}$ Ligand)]. All values are based on at least 50 snapshots from a single trajectory spaced by $10 \mathrm{ps}$ to avoid correlation between models.

\subsubsection{Binding affinity in vitro}

CYP2C9 was

immobilized to a planar

gold surface via a self-

assembled monolayer

(SAM) using Surface

Plasmon Resonance

(SPR) (Figure 2.3).
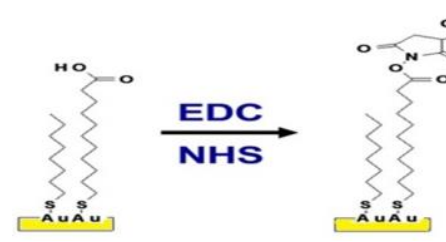

Figure 2.3. Self assembled monolayers of 3:1 OT:MUA were formed on planar gold. Carboxylic groups on MUA were activated by EDC/NHS to allow binding to $\mathrm{N}$-terminus methionine or surface exposed lysines of CYP2C9. 
Binding was assessed via surface plasmon resonance (SPR) showing reproducible immobilization levels of $70-200$ response units relative to the ethanolamine capped control surface (Figure 2.4). This low immobilization level was targeted in order to allow kinetic measurements of the system ${ }^{228,229}$.

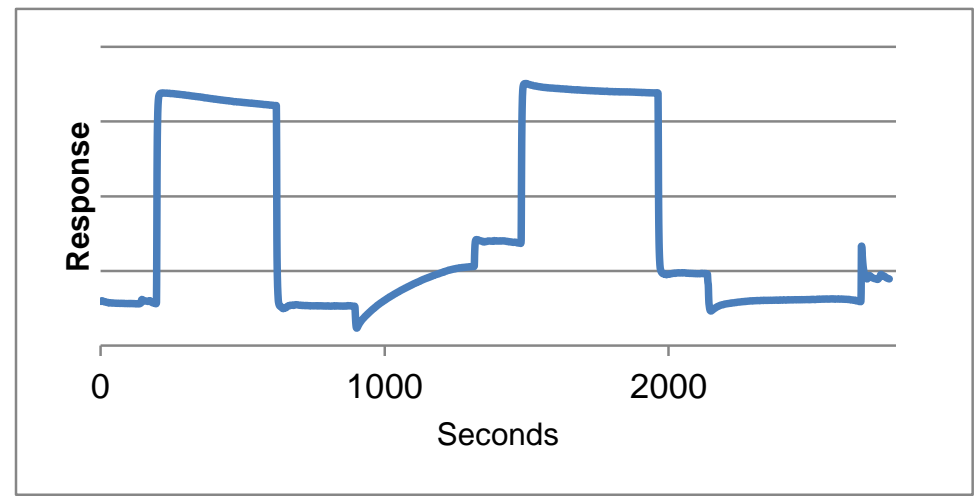

Figure 2.4. Representative sensorgram of CYP2C9 immobilization. Using a HEPES running buffer from time $=0 \mathrm{~s}$ the platform is subjected to EDC/NHS to activate the carboxylic acid groups in the SAM ( $t=$ $140 \mathrm{~s})$, CYP2C9 $(t=900 \mathrm{~s})$, ethanolamine to cap the unreacted NHS-esters ( $t=1490 \mathrm{~s}$ ), and finally washed with CTAB to remove any non-specifically binding $P 450$ molecules $(t=2140 \mathrm{~s})$. This procedure is followed in the control flow cell with the reversal of the ethanolamine and CYP2C9 injections to ensure both surfaces are subjected to identical conditions. The immobilized response was calculated from the final response level - baseline response of the experimental flow cell subtracting the values observed in the reference flow cell.

\section{Immobilized CYP2C9 (CYP2C9i) was exposed to soluble CYP2C9, CYP2D6 and CYP3A4}

to analytically determine equilibrium dissociation constants for each dimer pair. A control flow

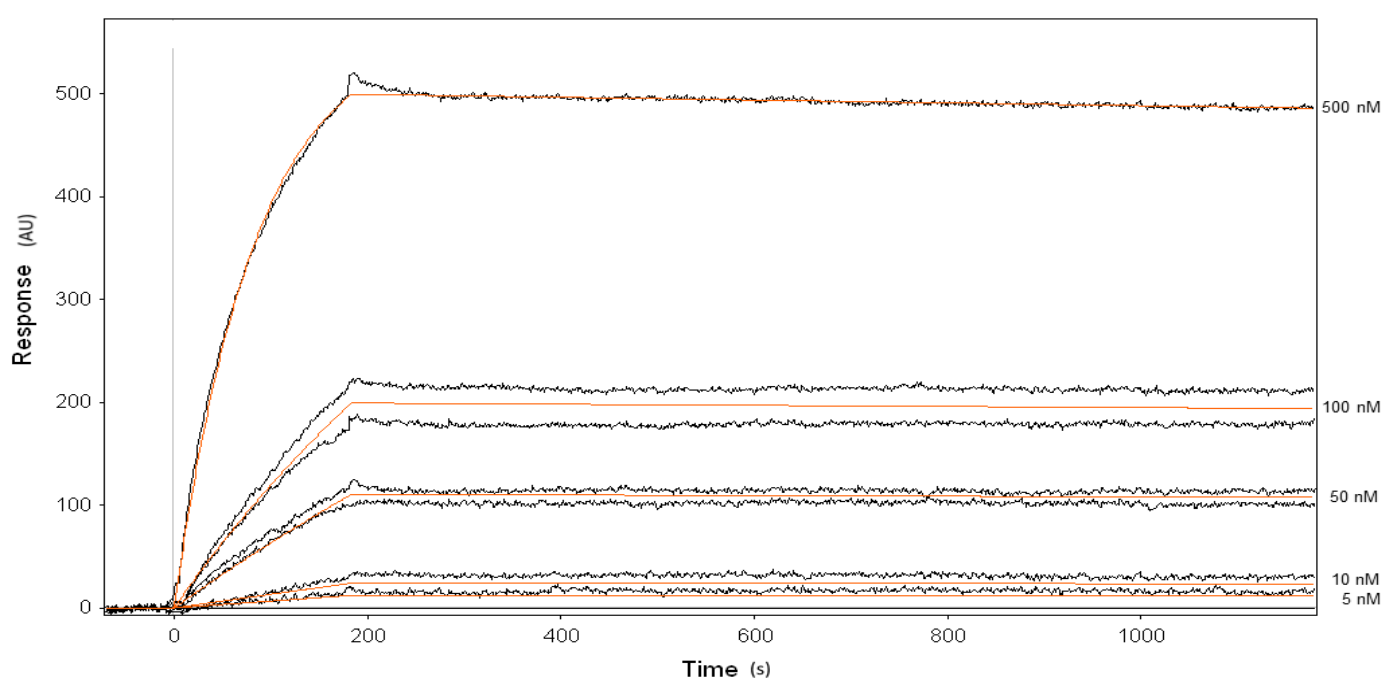

Figure 2.5. SPR sensorgram obtained from system comprised of soluble CYP2D6 binding to immobilized CYP2C9. Both the observed response (black) and the Scrubber 2.0 fitted lines (orange) are shown for CYP2D6 concentrations of $5,10,50,100$, and $500 \mathrm{nM}$. Concentrations 50 and $100 \mathrm{nM}$ were repeated to ensure reproducibility. CYP2D6 in PBS was flowed over the surface from $t=0-180 \mathrm{~s}$ and then PBS, only, for $t=180-900 \mathrm{~s}$ at a flow rate of $30 \mu \mathrm{L} / \mathrm{min}$. Immobilized CYP2C9 was bonded to the surface of the sample cell and ethanolamine capped SAM served as the control.in the control cell, at $30 \mu \mathrm{L} / \mathrm{min}$. 
cell was used to ensure that binding observations were not a result of non-specific interaction with the immobilization platform. A representative fitted sensorgram is shown in Figure 2.5. These results suggest that dimers are likely being formed as no other binding model adequately fit the data.

Binding equilibrium dissociation constants were calculated based on a 1:1 binding model. Ranked in order from strongest to weakest binding affinity are enzyme pairs CYP2C9CYP2D6, CYP2C9-CYP2C9, CYP2C9-CPR, and CYP2C9-CYP3A4 (Table 2.6).

Table 2.6 SPR Derived CYP2C9 equilibrium dissociation constants $\left(\mathrm{K}_{\mathrm{D}}\right)$. $^{\mathrm{a}}$

\begin{tabular}{|c|c|c|c|c|}
\hline Partner & CYP2D6 & CYP2C9 & CYP3A4 & CPR \\
\hline $\mathrm{K}_{\mathbf{D}}$ & $1.1 \pm 0.5 \mathrm{nM}$ & $2.6 \pm 1.0 \mathrm{nM}$ & $18.1 \pm 3.0 \mathrm{nM}$ & $7.3 \pm 2.2 \mathrm{nM}$ \\
\hline
\end{tabular}

${ }^{a} K_{D}$ values are for the binding of CYP2C9 with the indicated partner and were calculated by fitting the SPR sensorgrams with both the Biacore Evaluation or Scrubber 2.0 software and assuming 1:1 stoichiometry.

The binding affinity between immobilized CYP2C9 and CPR determined here is in the range of previously reported values for soluble CYP2C9-CPR binding which are $2.3+/-1.0 \mathrm{nM}^{230}$ and $32.8+/-0.2 \mathrm{nM}^{231}$.

\subsubsection{CYP2C9 effect on CYP2C9i-Mediated Flurbiprofen Metabolism}

We next investigated the effects of

P450-P450 interactions on metabolism

utilizing our immobilized platform. To

estimate the amount of immobilized enzyme

we relied upon our previous work ${ }^{106,158}$ and

our SPR immobilization to obtain a coverage

of $2 \mathrm{ng} / 48 \mathrm{~mm}^{2}$ on chips prepared in a

fashion identical to that used here. The effect

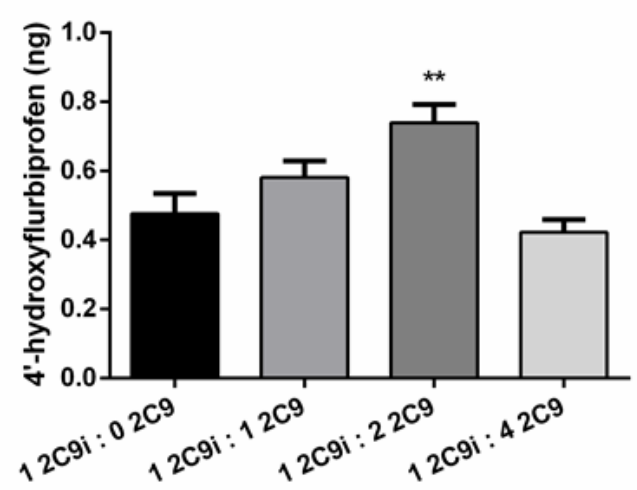

Figure 2.6. Effect of solution CYP2C9 on immobilized CYP2C9 (2C9i)-mediated metabolism of flurbiprofen at three ratios of 2C9i to lipidless CYP2C9 (2C9) and saturating CPR (P450(total): P450: 4 CPR, 1:4), incubated for 16 hours. A significant activation $(p<.01)$ is seen at a 2C9i:2C9 ratio of 1:2. Single-factor analysis of variance was used for statistical comparisons. 
of various amounts of CYP2C9 coincubated with CYP2C9i on flurbiprofen metabolism are presented in Figure 2.6.

Figure 2.6 shows the amounts of metabolite formed from CYP2C9i-mediated metabolism of flurbiprofen in the presence of three concentrations of lipidless CYP2C9 (2C9) in solution at a total P450:CPR ratio of 1:4. Metabolism by lipidless CYP2C9, alone, did not significantly differ from negative control (data not shown) and thus metabolite formation is due entirely to CYP2C9i. CPR was added prior to the addition of lipidless CYP2C9 followed by the addition of NADPH. When the ratio of CYP2C9i:CYP2C9 was 1:2 we observed significant activation of flurbiprofen metabolism. CYP2C9i -mediated metabolism of flurbiprofen was not significantly different from positive control at ratios above 1:2 (Figure 2.6).

\subsubsection{Effect of CYP3A4 and CYP2D6 on CYP2C9-mediated metabolism of flurbiprofen}

The effect of various amounts of CYP3A4 and CYP2D6 coincubated with CYP2C9i on metabolite formation from flurbiprofen are presented in Figures 2.7 and 2.8. The presence of CYP3A4 had no effect on CYP2C9-mediated metabolism of flurbiprofen when CYP2C9 was in solution with lipid (Figure 2.7a) or when CYP2C9 was immobilized (Figure 2.7b) with ratios of up to P450(total):CPR of 1:8. In both cases, CPR was added prior to the addition of CYP3A4.

a)

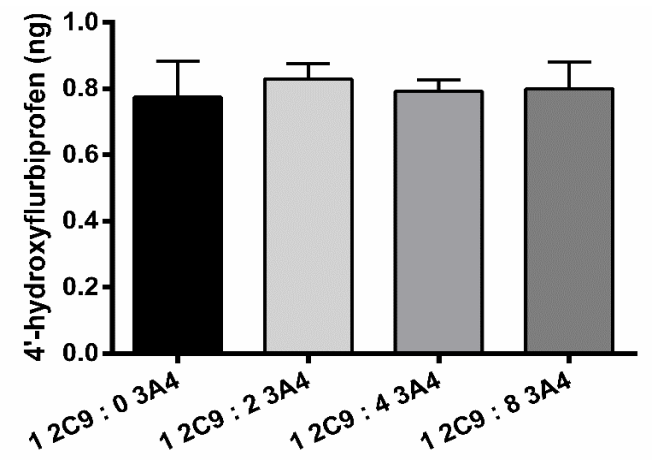

b)

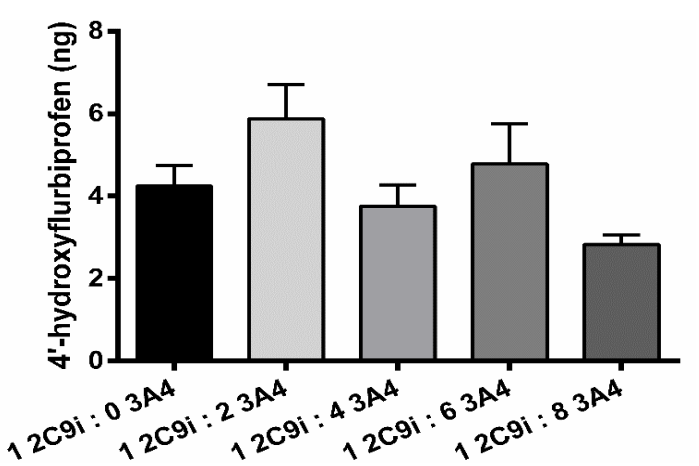

Figure 2.7. Effect of CYP3A4 on CYP2C9-mediated metabolism of flurbiprofen. a) Metabolite formation in solution incubations containing CYP3A4 and CYP2C9, reconstituted in lipid, and with saturating CPR (P450(total):CPR, 1:8), incubated for 16 hours. b) Metabolite formation in incubations containing CYP3A4 (solution), immobilized CYP2C9-mediated flurbiprofen metabolism at saturating CPR (P450(total):CPR, 1:8), incubated for 16 hours. Single-factor analysis of variance was used for statistical comparisons. 
In contrast, the presence of CYP2D6 significantly affected CYP2C9-mediated metabolism of flurbiprofen as suggested by the data shown in Figure 2.8. In particular, in solution, CYP2C9mediate metabolism of flurbiprofen was enhanced by the presence of CYP2D6 and in a concentration-dependent manner when CPR was present at saturating concentrations (P450(total):CPR, 1:8) and added prior to addition of CYP2D6 (Figure 2.8a). This activation saturated at a CYP2C9:CYP2D6 ratio of 1:4 and increased metabolite production by $\sim 400 \%$. In contrast, immobilized CYP2C9-mediated metabolism of flurbiprofen in the presence of CYP2D6 resulted in a concentration-dependent inhibition of CYP2C9-mediated metabolism of flurbiprofen

(Figure 2.8b). CYP2D6 inhibition of CYP2C9i metabolism saturated at $75 \%$ inhibition and did not change up to a ratio of CYP2C9i:CYP2D6 of 1:8.

a)

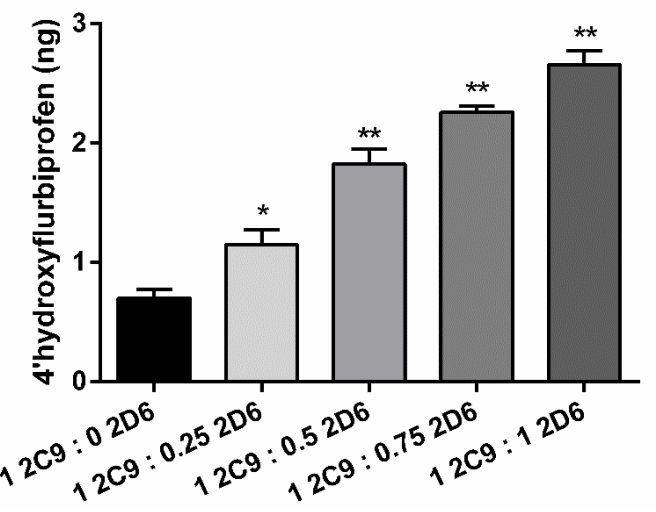

b)

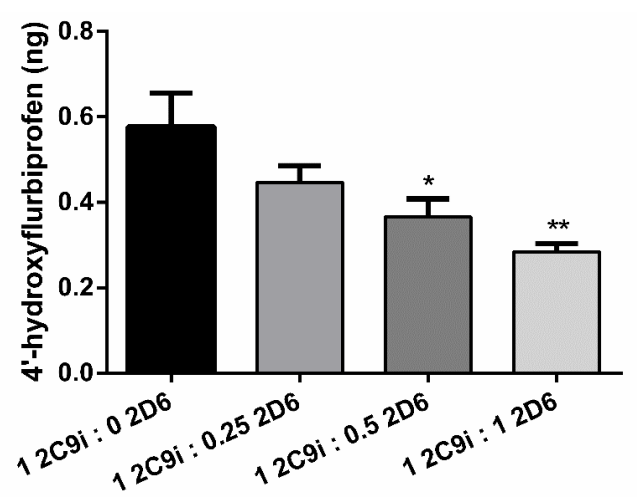

Figure 2.8. Effect of CYP2D6 on CYP2C9-mediated flurbiprofen metabolism. a) Effect of CYP2D6 on solution metabolism of CYP2C9 reconstituted in lipid with saturating CPR (1 total P450: 8 CPR), incubated for 16 hours. Significant activation ( $p<.05)$ is observed at a ratio of $12 C 9: 0.252 D 6$, and ( $p<.01)$ for ratios higher than $12 C 9: 0.252 D 6$. b) Effect of CYP2D6 on immobilized CYP2C9-mediated flurbiprofen metabolism at saturating CPR (1 total P450:8 CPR), incubated for 16 hours. Significant inhibition $(p<.05)$ is seen for 2C9i:2D6 ratios greater than 1:0.25. Single-factor analysis of variance was used for statistical comparisons of variance was used for statistical comparisons.

\subsubsection{Influence of the order of addition of enzymes on CYP2C9-mediated metabolism}

Figure 2.9 displays the amount of metabolite formed as a function of the order of addition of either CYP3A4 or CYP2D6 P450 and CPR from CYP2C9-mediated metabolite production. 
Figure 2.9a shows that there is no effect of CYP3A4 on CYP2C9-mediated metabolism when CPR is added first and allowed to preincubate which is in agreement with the results presented in Figure 2.6a. In contrast, when CYP3A4 is allowed to preincubate with CYP2C9, a significant inhibition up to 34\%, of CYP2C9-mediated metabolism of flurbiprofen at a CYP2C9:CYP3A4 ratio of $1: 2$ is observed. The effect on metabolite production by CYP2D6 in shown in Figure 2.9b and shows that the presence of CYP2D6 strongly inhibits CYP2C9 metabolism (up to 98\%) regardless of the order the components are added prior to initiating the incubation. There is no significant difference between metabolite production at the 1 CYP2C9 : 2 CYP2D6 ratio when CPR is added before or after CYP2D6.

a)

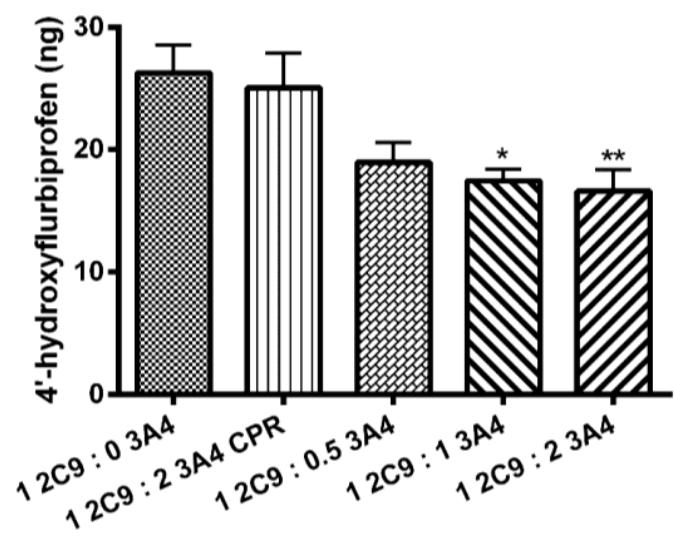

b)

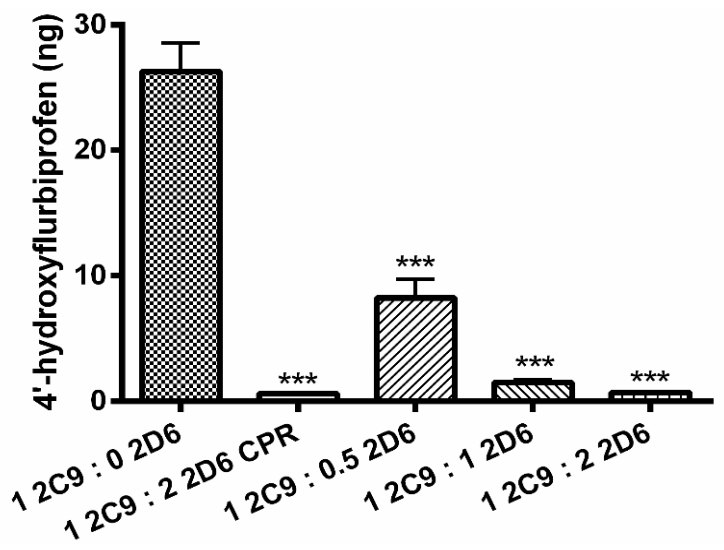

Figure 2.9. Order of addition of CPR and CYP3A4 or CYP2D6 effect on CYP2C9 mediated flurbiprofen metabolism. a) Effect of CYP3A4 on solution metabolism of CYP2C9 reconstituted in lipid with sub-saturating CPR (1 total P450: 1.5 CPR), incubated for 1 hour. $12 C 9: 23 A 4$ CPR represents preincubation of CYP2C9 and CPR for 5 minutes prior to CYP3A4 addition. All other columns represent CYP3A4 preincubation for 5 minutes prior to addition of CPR. A significant inhibition at $2 C 9: 3 A 4$ ratio of $1: 1(p<.05)$ and $(p<.01)$ at a ratio of 1:2 is seen in the presence of preincubated CYP3A4, but not when CPR is preincubated. b) Effect of CYP2D6 on solution metabolism of CYP2C9 reconstituted in lipid with sub-saturating CPR (1 total P450:1.5 CPR), incubated for 1 hour. $12 C 9: 22 D 6 C P R$ represents preincubation of CYP2C9 and CPR for 5 minutes prior to CYP2D6 addition. All other columns represent CYP2D6 preincubation for 5 minutes prior to addition of CPR. A significant inhibition $(p<.001)$ is seen in the presence of CYP2D6. There was no significant difference between preincubating the CPR or CYP2D6 with CYP2C9 at the ratio of $12 C 9: 22 D 6$. Singlefactor analysis of variance was used for statistical comparisons.

\subsection{Discussion}

Protein aggregation between P450s in solution has been shown to occur and can activate or inhibit P450-mediated metabolism. Previous work has demonstrated that the consequences of P450-P450 interactions are not universal, and some specificity between partners may be 
involved $67,74,81$. In the present study, we show the formation of both homomeric (CYP2C9CYP2C9) and heteromeric (CYP2C9-CYP3A4, CYP2C9-CYP2D6, CYP2C9-CPR) P450 complexes with different binding affinities. We reveal for the first time activation of CYP2C9 metabolism by homomeric complex formation. Furthermore, we show that controlling the aggregation state of CYP2C9 through immobilization changes the effect of P450-P450 interactions has on metabolism. Lastly, we show that order of addition and concentration of CPR modulates inhibition of CYP2C9-mediated metabolism by CYP2D6 and CYP3A4.

Computational modeling was used as a tool to predict the conformation of dimer formation and how the complex may affect P450-P450 modulation of metabolism. Models were generated using PDB ID's 10 G5 and 1R90 that represent two different conformations of the CYP2C9 enzyme that may be adopted during enzyme-mediated metabolism, the closed and partially open forms. The data shown in Table 2 indicates that dimerization can involve the solvent or substrate channels and the CPR binding site in about $25 \%$ and $7.5 \%$, respectively, of the predicted models. We investigated both the closed conformation of CYP2C9 (1OG5) and partially open conformation (1R90), as they both exist in vitro and in vivo upon substrate binding and release during the catalytic cycle. The closed conformation involved the solvent channel region more frequently, while the partially open conformation involved all regions of interest uniformly.

In particular, metabolism is more likely to be affected due to dimerization occurring at the solvent channel for CYP2C9 and CYP3A4, thus changing binding or turnover rate, as compared to CYP2D6. In the dimer models generated, a binding conformation wherein the CPR binding site was aligned planar with the partner enzyme occurred in $12 \%$ of the models, or about 1.6 times more frequently than when the CPR site was blocked, suggesting that altered affinity for CPR enzyme could be an important contributor to changes in enzyme metabolism (Table 2.4). MMGBSA calculations were conducted to ensure the predicted GRAMMX dimers were 
favorable and to compare binding between the different complexes. The results demonstrate that each dimer is energetically favorable, and we observe the most favorable $\Delta G$ for CYP2C9CPR (Table 2.5). The favorable $\Delta \mathrm{G}$ observed for CYP2C9-CPR, a known 1:1 complex, helps validate our GRAMMX results.

Currently, there are very few experimental methods that measure or demonstrate P450P450 complex formation, despite the large amount of evidence of P450 interactions. Thus far, co-immunoprecipitation studies ${ }^{67,78}$ and fluorescently-labeled P450 fluorescent energy transfer studies ${ }^{80,195}$ confirm P450-P450 complex formation. Optical sensors, such as SPR, have been used previously to measure complex formation between P450cam and putidaredoxin ${ }^{180}$. In the present study we used SPR to measure binding of analyte P450s to immobilized CYP2C9 in real-time. CYP2C9 was immobilized and the binding partner flowed over in the absence of substrate, conditions that favor the partially open conformation. The $K_{D}$ values determined for the binding of CPR to CYP2C9 is in line with previously reported values by Wei et al. 2003 and Locuson et al. 2007, demonstrating the likely validity and accuracy of this methodology. The high affinity binding which occurs between CYP2C9-CYP2C9 and CYP2C9-CYP2D6 is likely relevant to the results of metabolism assays as they bind to one another more strongly than either does to CPR (Table 2.6). We also show that CYP3A4 forms a heteromeric complex with CYP2C9, but its $K_{D}, 2.5$ times that of CPR (Table 2.6), and suggests CYP3A4 competition for the CPR binding site is unlikely to factor into metabolism effects; however, this does not preclude the possibility of CYP3A4 altering CYP2C9's affinity for CPR. Our results also offer an in vitro validation of our modeling results showing good agreement with our predicted binding between P450s, with the only deviation being that CYP2C9-CPR binding is predicted to be slightly more favorable than CYP2C9-CYP2D6. Furthermore, the immobilization scheme is the same used for our metabolism studies, allowing for direct interpretation of complex formation effect on metabolism. 
P450 aggregation can affect metabolism via altered CPR affinity, substrate binding, or turnover rate ${ }^{81}$. Altered CPR affinity can occur through blocking CPR binding sites and preventing association and inhibiting metabolism. Alternatively, a planar alignment of CPR binding sites with dimer partners may increase CPR binding tightness and thereby enhance metabolism. In the present study, we use our controlled immobilization platform to show for the first time the activation of immobilized CYP2C9 metabolism through homomeric complex formation (Figure 2.6). It is possible that dimerization of CYP2C9 restores activity to CYP2C9 that is in solution (lipid free); however, if this was the case we would expect the metabolite generation to saturate at the highest level. Instead, we note a decrease back to the control at ratios greater than 1:2 (Figure 2.6). Increased affinity for CPR is one possibility for activation given the aligned CPR binding sites in one of our predicted homodimers (Table 2.4). Another factor could be interaction with solvent access channels promoting substrate binding and exit (Table 2.2) ${ }^{232}$. The lack of activation at increased ratios might be due to larger aggregate formation that alters the synergistic kinetics. However, the SPR model suggests that only dimer formation occurs so further study is needed to evaluate the source of this effect.

In solution, it has been shown that protein-protein interactions between CYP2C9 and CYP3A4 yield a protein dose-dependent inhibition of CYP2C9 metabolism when incubated with CYP3A4 prior to CPR addition ${ }^{78}$. Here we show CYP2C9 pre-incubation with CPR does not lead to inhibition by CYP3A4 with solution or immobilized CYP2C9 (Figure 2.7) regardless of CPR concentration (Figure 2.9a). Furthermore, the work by Subramanian et al. (2010) showed that the CYP2C9-CYP3A4 interaction could be eliminated by truncation of the $\mathrm{N}$-terminus of either binding partner. We show however, that if both P450s are truncated interaction can still occur (Table 2.6), and that this binding can result in decreased solution CYP2C9 metabolism when pre-incubated with CYP3A4 (Figure 2.9a). The order of addition of protein binding partners and cofactors has been shown to be important when evaluating the effects of P450- 
P450 interactions ${ }^{194,233}$. The order of addition effect on CYP3A4 inhibition points towards the formation of a quasi-irreversible heteromeric complex (Figure 2.9a).

Studies have also shown that there is a protein concentration-dependent inhibition of CYP2C9 metabolism when co-incubated with CYP2D6 at sub-saturating CPR concentrations 194. Interestingly, we note drastically different effects of CYP2D6 on CYP2C9 metabolism depending on study parameters. Our result of 94\% inhibition of CYP2C9 in solution by CYP2D6 at a $1: 1$ ratio (Figure $2.9 b$ ) is in reasonable agreement with the $50-80 \%$ reported previously ${ }^{194}$. The difference in maximum inhibition could possibly be due to differences in lipid:protein ratios between the two studies. However, we show that at saturating CPR concentrations CYP2C9 in solution metabolism is activated by CYP2D6 (Figure 2.8a). Even more compelling, is that when CYP2C9 is immobilized, CYP2D6 is once again inhibiting despite that excess CPR is being allowed to preincubate with the immobilized CYP2C9 (Figure 2.8b). Competition for CPR is unlikely to be a factor as the reported $K_{D}$ for CYP2D6-CPR interaction (20 nM) ${ }^{234}$ is almost three times that of CYP2C9-CPR (Table 2.6).

One possible explanation for the solution metabolism results could be a dual effect of CYP2D6 on CYP2C9 metabolism. If the CYP2D6-CYP2C9 complex has a faster substrate turnover rate, but lower CPR affinity, we would expect to see activation of metabolism when CPR is well in excess and the faster turnover rate dominates the kinetics but inhibition when CPR is sub-saturating and electron transfer becomes the limiting step. Another factor to consider is the difference between solution and immobilized platform results (Figure 2.8). Immobilization of CYP2C9 controls its aggregation, with the majority being monomeric ${ }^{158,204}$. Therefore, the inhibition in CYP2C9 metabolism could be a result of the lack of competition of homomeric complex (CYP2C9-CYP2C9) formation with heteromeric complex (CYP2C9CYP2D6) formation, evidenced by the close $K_{D}$ values for both interactions (Table 2.6). On the immobilized platform the binding modes and conformations may also be limited or otherwise 
different compared to solution and CYP2D6 may prevent CPR from associating with CYP2C9, thus limiting metabolism.

The current study demonstrates the utilization of an immobilized platform to better understand P450-P450 interactions. Being able to immobilize an enzymatically active P450, we are able to study both P450-P450 complex formation and the resultant metabolic modulation. We show that CYP2C9 forms both homomeric and heteromeric complexes, and that these complexes have the ability to activate or inhibit CYP2C9 metabolism. Future studies using this platform can further explain intricacies of these interactions, and can be expanded to probe whether effects are due to changes in CPR affinity or substrate turnover.

\subsection{Contributions}

Molecular modeling data, computationally predicted dimers and binding free energy in silico, was provided by Dr. Katherine M. Hickey. SPR binding constants for CYP2C9-CYP2D6 and CYP2C9CYP3A4 were provided by Dr. Lance A. Wollenberg and Dr. Katherine M. Hickey respectively. 


\section{Chapter 3}

\section{Nanoscale electron transport measurements of immobilized cytochrome $\mathbf{P} 450$ proteins}

Adapted from Bostick, C.D., Gannett, P.M., Flora, D.R., Tracy, T.S., and Lederman, David. Nanoscale Electron Transfer Measurements of Immobilized Cytochrome P450 Proteins. Nanotechnology 2015, 26(15), 155102 


\subsection{Introduction}

The electron transfer (ET) properties of heme-thiolate monooxygenases have been extensively studied and, as a result, the ET process for a subset of these proteins has been well characterized $^{127}$. One example of such a protein is cytochrome $\mathrm{c}$, which has become a model for studying biological $\mathrm{ET}^{128}$. There have been comparatively fewer studies of electron transfer on monooxygenases involved in metabolic processes. The cytochrome P450s (P450s) are monooxygenases and part of a large family of heme containing proteins found primarily in the liver where they are bound to the smooth endoplasmic reticulum ${ }^{11}$. The main function of P450s is to metabolize substrates into more water-soluble and readily excretable forms, primarily via oxidation (e.g., insertion of an oxygen atom, typically into a C-H bond, to form a hydroxylated product or demethylation). The transfer of an electron from a donor (cytochrome P450 reductase, CPR) to the P450 heme group is the controlling step for the initiation of the catalytic cycle. ${ }^{15} \mathrm{~A}$ better understanding of ET in P450s would allow exploitation of their unique properties for use in new biomedical applications, for example, in bioreactors for bio-transformations/metabolic engineering ${ }^{86,87,106}$, biosensors for personal drug monitoring ${ }^{129,149,161}$,or bioremediation ${ }^{91}$.

Several factors have been suggested to affect ET, such as substrate binding and protein aggregation. Substrate binding has been observed to alter the spin state of the heme iron in P450s ${ }^{44}$. Spin state has been measured using absorption spectroscopy and some degree of correlation has been observed between changes in spin state and metabolic activity ${ }^{17,30}$. Type II ligands to P450 enzymes, are often inhibitors and not metabolized and have no effect on spin state, or stabilize the enzyme in the low spin state. ${ }^{44}$ Moreover, there are many substrates, such as dapsone, that alter the spin state very little, but are nevertheless metabolized. The reduction of heme can also occur in the absence of substrate in some P450s, and equal reduction rates between low and high spin heme species have been measured ${ }^{16}$. This suggests that other factors may be modulating ET other than spin-state changes caused by substrate binding. 
P450s are known to aggregate, forming homo- and hetero-oligomers in solution, and a range of effects from these interactions can occur, such as alteration in catalytic rate or degree of binding to the binding partner $\mathrm{CPR}^{64-66,68,194,196}$. Attempts to prevent this aggregation using detergents, salts, and lipids have been shown to affect $\mathrm{ET}^{57,72,235}$. Due to these difficulties, it has not been possible to determine, how oligomer formation alters metabolism, and it is very possible that aggregation affects ET. A related factor is whether CPR binding to P450s, an obligatory step in the P450 catalytic cycle, alters ET. For example, it is possible that the binding of CPR to a P450 may induce a conformational change that facilitates $E T^{147,236-238}$. It is also possible that substrate binding causes conformational changes that affect ET from CPR to $\mathrm{P} 450^{30,239}$, and by combined processes may also modulate ET.

The literature suggests that the binding of substrates may be key for facilitating ET in P450s ${ }^{182,240,241}$, and a correlation between the rate of substrate metabolism and the ease of ET has been proposed ${ }^{159,172,242,243}$. One approach used to explore this issue has been to immobilize P450 to an electrode in an attempt to achieve direct electron supply from the redox electrode to the redox active group of the $\mathrm{P} 450^{98}$. A variety of techniques have been used to achieve immobilization, including the direct wiring of a flavin cofactor to the heme ${ }^{244}$, confining P450s inside thin films on electrodes ${ }^{141}$, and tethering of modified P450s ${ }^{245}$. These attachment procedures have permitted the use of electrochemical methods, including cyclic voltammetry, square wave voltammetry, and amperometry, thus providing a way to measure critical parameters relevant to the redox processes in the proteins (e.g., the redox potential $E^{\circ}$ ) under a variety of conditions $^{135}$. However, these electrochemical measurements tend to alter protein conformation and function ${ }^{182}$, cause interactions of the heme with the electrode ${ }^{137}$, and there is ambiguity of the aggregation state of the enzyme as a consequence of immobilization. Consequently, the results have not established a clear correlation between metabolism or metabolic rate and $\mathrm{ET}^{242}$.

Recently, immobilization of unmodified P450s on electrodes using various self-assembled monolayers (SAM) has allowed the use of electrochemistry approaches to study P450s that retain 
their metabolic activity ${ }^{106,149,161}$. Nanoscale approaches have also been used to probe single enzyme electron transport (ETp), including scanning probe microscopy ${ }^{162}$, mechanical break junctions $^{166}$, and conductive probe atomic force microscopy (CPAFM ${ }^{246}$. We recently developed a methodology to measure the properties of non-aggregated P450s using CPAFM that allows the study of the same individual P450 enzyme in response to different bound substrates ${ }^{46}$. In this approach, electron beam lithography is used to pattern four $21 \times 21$ gold nanopillar arrays on doped silicon. The lateral dimensions of the nanopillars are in the range of 20 to $40 \mathrm{~nm}$, allowing isolation of the P450 of interest through bonding to a SAM bonded to the gold nanopillar. Here, the SAM was comprised of $\omega$-thioundecanoic acid which selectively bonds to gold and not silicon when dissolved in ethanol ${ }^{152}$, ensuring attachment of the P450 to gold pillars and not the surrounding silicon. Other laboratories have made similar arrays using analogous methods ${ }^{170}$. Using this indexed array of gold nanopillars unmodified cytochrome P450 2C9 (CYP2C9) was selectively immobilized, the electrical conductance measured with CPAFM on the same enzyme molecule under different conditions, and a correlation between ET, ETp, and enzyme metabolic activity was found ${ }^{46}$.

Here we describe important advances in our CPAFM measurement technique. By measuring changes in pillar height prior to and after attachment, we confirm that the nanopillars have single layer P450 coverage, and not an aggregated enzyme. In addition, we demonstrate how the AFM tip force modulates the conductance of the proteins on the gold nanopillars. Finally, we demonstrate that the CYP2C9 inhibitor sulfaphenazole reduces $\mathrm{ET}$, in agreement with our previous results with another inhibitor (aniline) $)^{46}$.

\subsection{Materials and Methods}

\subsubsection{General}

All chemicals were purchased from Sigma Aldrich (Milwaukee, WI) and were of analytical reagent grade and used without further purification unless otherwise noted. Acetone, isopropanol, methyl isobutyl ketone, mercaptoundecanoic acid (MUA), 8-octanethiol (OT), N-((3- 
dimethylamino)propyl)-N-ethylcarbodiimide hydrochloride (EDC), N-hydroxysulfosuccinimide (NHS), flurbiprofen, dapsone, and potassium phosphate-buffer (Kpi, $40 \mathrm{mM}, \mathrm{pH}$ 7.4, prepared from potassium mono- and dibasic phosphate). Ethanol (100\%) was purchased from PharmcoAaper (Brookfield, CT). The 300 MIF developers, 495K poly(methyl methacrylate) (PMMA) and 950K PMMA, were obtained from Micro Chem (Newton, MA). Boron-doped [100] silicon wafers were obtained from University Wafer (South Boston, MA). AZ 5214 photoresist was obtained from AZ Electronic Materials (Capitol Scientific, Austin, TX). Cytochrome P450 2C9 enzyme (CYP2C9) was prepared by expression in an E. coli system, isolated, and purified as described previously ${ }^{247}$. Deionized water was obtained from a Nanopure Ultrapure (Thermo Scientific, Waltham, MA) water system and had a resistivity of $18.2 \mathrm{M} \Omega-\mathrm{cm}$.

\subsubsection{Chip Cleaning and Preparation}

Silicon wafers were cleaned by immersion in Buffered Oxide Etch (BOE) 1:10 hydrofluoric acid (49\%):deionized water solution for $3 \mathrm{~min}$ and rinsed in a cascading water bath for $10 \mathrm{~min}$. The wafers were then blown dry with nitrogen, and immediately spin coated with PMMA to prevent silicon oxidation. The wafers were placed in a Laurell WS-400B-6NPP/LITE spin-coater (North Wales, PA), and spincoated with 495K PMMA (4\% in anisole) (5000 rpm, $30 \mathrm{~s}$ ), placed on a hot plate $\left(180^{\circ} \mathrm{C}, 2 \mathrm{~min}\right)$ to evaporate solvent, and cooled to room temperature. Samples were then spin-coated with 950K PMMA (4\% in anisole) (5000 rpm, $30 \mathrm{~s})$, placed on a hot plate $\left(180^{\circ} \mathrm{C}, 2\right.$ $\mathrm{min}$ ) to evaporate solvent, and cooled to room temperature.

\subsubsection{Electron Beam Lithography}

Electron beam lithography was then performed to pattern the PMMA using a JEOL JSM-7600F field emission analytical scanning electron microscope (Tokyo, Japan) equipped with Nanometer Pattern Generating System software from JC Nabity Lithography Systems (Bozeman, MT). The working conditions were chamber pressure $\leq 9.6 \times 10^{-5}$ Torr, electron beam accelerating voltage $30.0 \mathrm{kV}$, working distance $8.0 \mathrm{~mm}$, and probe current $40-45 \mathrm{pA}$. To index the nanopillars, a fiducial marker was made via a diagonal scratch, from one corner, on the PMMA. After focusing, 
the pattern was written under software control at the end of the fiducial scratch. Upon completion of electron beam lithography, samples were developed by rinsing in a solution of 1:3 methyl isobutyl ketone:isopropyl alcohol (70 s), then in $100 \%$ isopropyl alcohol (20 s), and then deionized water (10 s).

\subsubsection{Electron Beam Evaporation}

A Temescal BJD-2000 system (Edwards Vacuum, Phoenix, AZ) with an Inficon XTC/2 deposition controller (East Syracuse, NY) was used for metal evaporation. Chamber pressures were $\leq 1.0 \times 10^{-5}$ Torr. Samples were rotated $(3 \mathrm{rpm})$ and monitored for metal thickness using a crystal monitor with gold-coated $6 \mathrm{MHz}$ quartz piezoelectric crystals (Kurt J. Lesker Co., Clairton, PA). Rates of $0.3-0.5 \AA /$ s were maintained during the deposition of a titanium adhesion layer (2 $\mathrm{nm}$ ) and a gold layer (15 $\mathrm{nm})$. After deposition, samples were cooled to room temperature before being removed from the chamber. Lift-off of the photoresist was performed by scoring chips with a razor blade, on opposite side of pillar writing, to allow acetone to reach under metal and PMMA layer. Then samples were placed in warm acetone $\left(60-70{ }^{\circ} \mathrm{C}\right)$ for 45 minutes to an hour, with periodic spraying with RT acetone from squirt bottle. SEM images of the nanopillar arrays are shown in Figure 3.1. Steps of PMMA spin coating, metal deposition, and lift-off were all conducted in a 24 hour period to yield best results.

\subsubsection{Scanning Electron Microscopy and Atomic Force Microscopy Imaging}

SEM imaging was performed using a JEOL JSM-7600F (Peabody, MA) field emission analytical scanning electron microscope with a pressure inside the chamber of $\leq 9.6 \times 10^{-5}$ Torr. AFM imaging was performed using an Asylum MFP 3D-BIO AFM (Santa Barbara, CA) in tapping mode using Asyelec-01 silicon tips (Asylum Research, Santa Barbara, CA). Height images were taken in tapping mode to minimize tip effects. Gwyddion version 2.37 (Gwyddion.net) was used for analysis of taping mode height images ${ }^{248}$. Height analysis was done by using a grain threshold to highlight the pillars and obtain a distribution of pillar height. This distribution was then fit to a Gaussian model to obtain a mean pillar height from the peak center. 


\subsubsection{Immobilization of CYP2C9 to Gold via Self Assembled Monolayer}

Samples were washed in acetone, methanol, and deionized water, each, for $5 \mathrm{~min}$, washed with ethanol, and immersed in an ethanolic solution of OT (10 mM) and MUA (2.5 mM) (18 h), and rinsed with ethanol and then $40 \mathrm{mM}$ potassium phosphate buffer $\mathrm{pH} 7$ (Kpi), three times each. Samples were immersed in Kpi containing EDC $(2 \mathrm{mM})$ and NHS $(5 \mathrm{mM})$ for $2 \mathrm{~h}$, and then immersed in a Kpi solution containing CYP2C9 $(50 \mathrm{nM})$, flurbiprofen $(40 \mu \mathrm{M})$, and dapsone (40 $\mu \mathrm{M})$ for $24 \mathrm{~h}$. After the CYP2C9 was attached, the samples were rinsed with $3.9 \mathrm{mM}$ Cetyltrimethylammonium Bromide (CTAB) for 5 minutes to remove non-specific binding, then Kpi, and then blown dry with nitrogen.

\subsubsection{Conductive Probe Atomic Force Microscopy Measurements}

Prior to CPAFM measurements, the tip cantilever spring constants were measured in accordance with the manufacturer's instructions on freshly stripped mica for all RMN 25Pt300B tips (Rocky Mountain Nanotechnology, Salt Lake City, UT) used. Spring constant calculations were used to apply the same force or modulation of force during all CPAFM measurements. Fiducial scratches were used to orient our samples to take measurements on the same nanopillars for each CPAFM measurement. CPAFM scans were taken in-air by starting the bias at $0 \mathrm{~V}$, moving linearly to maximum bias ( 3 to $4 \mathrm{~V}$ ), then linearly to minimum bias $(-3$ to $-4 \mathrm{~V})$, and finally returning to $0 \mathrm{~V}$. Curves shown for each sample are the average of at least five scans taken from the maximum to the minimum voltage cycle. Substrates were applied by immersing chips with immobilized CYP2C9 in a Kpi solution containing $40 \mu \mathrm{M}$ of flurbiprofen and $40 \mu \mathrm{M}$ dapsone, or $40 \mu \mathrm{M}$ sulfaphenazole, and then blown dry with nitrogen. Between CPAFM experiments, samples were rinsed in deionized water for at least $1 \mathrm{~h}$ to wash small molecules (flurbiprofen, dapsone, or sulfaphenazole) from the enzyme. All processes were performed in an argon atmosphere at room temperature.

\subsection{Results and Discussion}


A unique aspect of the nanopillar array is that it allows for sequential measurements under different conditions to be conducted on the same bonded CYP2C9 molecule. This, in part, was made possible by using a scratch as a fiducial marker which allowed us to find the same nanopillar with the same CYP2C9 molecule in subsequent studies. Scanning electron microscopy (SEM) images of the nanopillar platform are shown in Figure 3.1. The images show that each nanopillar was between 20 and $40 \mathrm{~nm}$ in diameter. CYP2C9 was attached to the nanopillars as described in Jett et $a^{46}$. Briefly, a self-assembled monolayer was bonded to the gold nanopillars containing $\omega$-mercaptoundecanoic acid. The carboxyl groups of the SAM were activated toward amide formation such that the CYP2C9 was bonded to the gold nanopillars through the SAM. Bonding to the SAM can occur through the N-terminus or any surface accessible lysine of CYP2C9. While it has been suggested that the orientation of the heme (controlled by the orientation of the enzyme with respect to the pillar) plays an important role in $\mathrm{ET}^{249}$, the reproducibility of our results indicate that our enzyme is either able to adopt a similar conformation across pillars or that heme orientation is inconsequential. Previous cyclic voltammetry studies done by our lab utilizing the same attachment scheme of CYP2C9 on a gold film revealed a peak width characteristic of a prevalent orientation of our bonded enzyme ${ }^{106}$, and we hypothesize attachment is on a lysine near the $\mathrm{N}$-terminus due to $\mathrm{pKa}$ and steric constraints ${ }^{158}$. In addition, because of this experimental construct minimal changes are expected in conformation when measuring the same pillar under different conditions (except for changes caused by changes in force). One question about this immobilized system is whether the CYP2C9 that is bonded to the gold is still active, and able to metabolize substrate. Previous work in our laboratory and the current work have demonstrated that this system maintains the ability to carry out CYP2C9-mediated metabolism of compounds known to be substrates, when endogenous co-factors and protein partners (i.e., NADPH and CPR) are present ${ }^{158}$. Therefore, the ETp studies conducted here are on immobilized CYP2C9 proteins that retain their in vivo activity. 

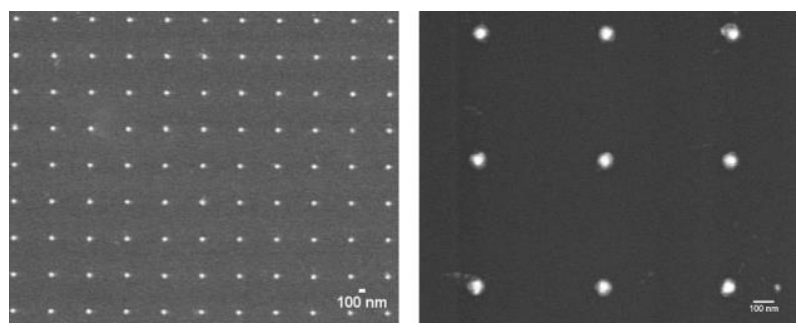

Figure 3.1. SEM image showing a section of one of the four nanopillar arrays created by electron beam lithography (left) and zoomed in to show the size of the nanopillars (right).

Since aggregation of the P450 proteins may alter the ET and ETp processes, to obtain unconfounded information about the P450 ET process, aggregation effects need to be identified or removed. Thus, studies like those herein which employ isolated P450 can be used to minimize aggregation and its effect on ET. The nanopillar lateral dimensions, on average, are approximately $30 \mathrm{~nm}$ and the diameter of a single CYP2C9 enzyme is approximately $6 \mathrm{~nm}^{6}$. Based on previous studies in our lab, we expect approximately $50 \%$ coverage depending on the CYP2C9 concentration used, on the top of each nanopillar. Thus, it is expected that a maximum of 13 CYP2C9 molecules could bind to the top of a nanopillar ${ }^{158}$.

Monolayer attachment would show that we are not measuring through multiple enzymes, and leave only the possibility of lateral enzyme interaction(s). Changes in pillar height were used to evaluate attachment and aggregation of CYP2C9. While conductive studies were completed in contact mode, height images were obtained in tapping mode to avoid possibly moving bonded CYP2C9 off the pillar top during raster scanning ${ }^{250}$. Maximal z-axis resolution of AFM has been shown to be $\sim 0.1 \mathrm{~nm}^{188}$, which is more than sufficient for distinguishing between a single CYP2C9 or two CYP2C9s stacked on top of another. Parameters to obtain monolayer coverage were evaluated on gold films, and height was assessed by scratching a small area clean in contact mode at a high force $(160 \mathrm{nN})$, and re-imaging in tapping mode with a new tip ${ }^{251}$. This showed a height change of $\sim 1.1 \mathrm{~nm}$ for SAM, and $\sim 7 \mathrm{~nm}$ for SAM/CYP2C9, which is in good agreement with monolayer coverage (Figure 3.2). 
a)

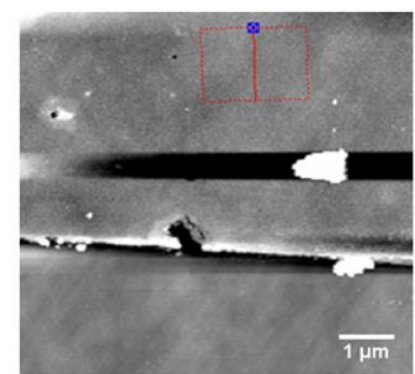

c)

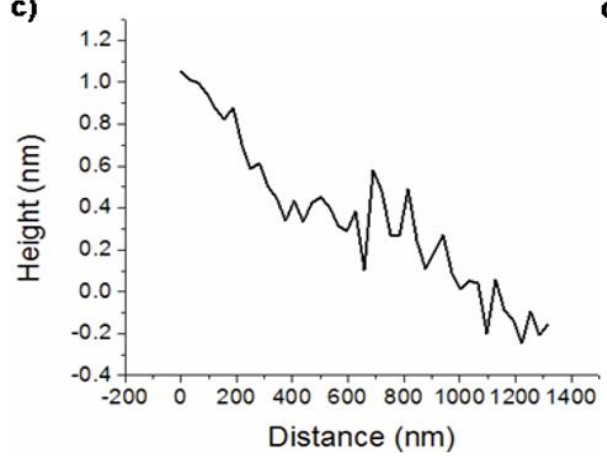

b)

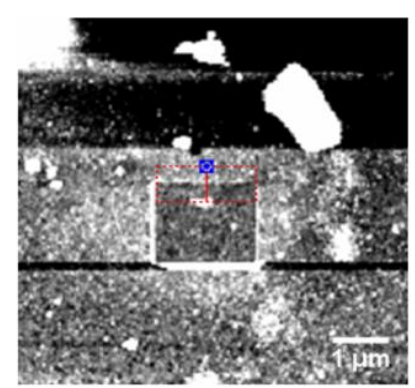

d)

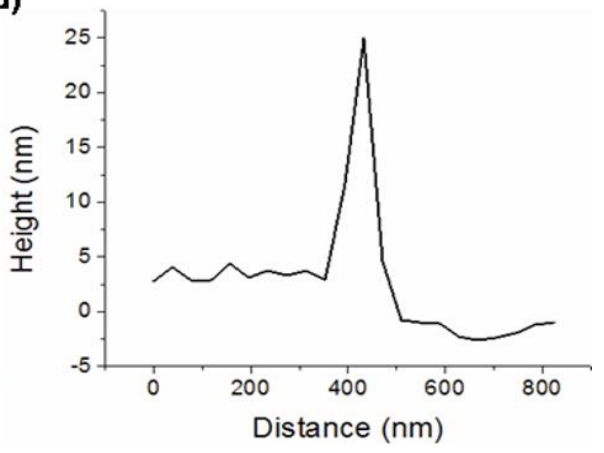

Figure 3.2. a) AFM tapping mode height image of Au film with attached SAM, and b) with attached SAM/CYP2C9 after a small $2 \mu m \times 2 \mu m$ section was scratched clean by contact mode imaging at high force $(160 \mathrm{nN})$. A new tip was used to obtain the tapping mode image from the one used to perform the contact mode scratch cleaning. c) Section graph, red box in a), of the Au film with attached SAM across the contact and non-contacted cleaned area showing a height difference of $\sim 1.1 \mathrm{~nm}$. d) Section graph, red box in b), of the Au film with attached SAM/CYP2C9 across the contact and non-contact cleaned area showing a height difference of $\sim 7 \mathrm{~nm}$. Data from the other sides of the contact cleaned area are in good agreement with those reported in c) and d) (data not shown). 
Figure 3.3 depicts an AFM height image of our platform with pillars highlighted through grain analysis in Gwyddion software. A height threshold was chosen to select only for the gold pillars and not the silicon surface, as our attachment scheme will only allow bonding of our enzyme to gold through amide bond formation with the SAM. A height distribution was extracted for an entire array (441 nanopillars) and fit to a Gaussian line shape to find the peak center. An overlay of the height distributions for bare gold pillars and

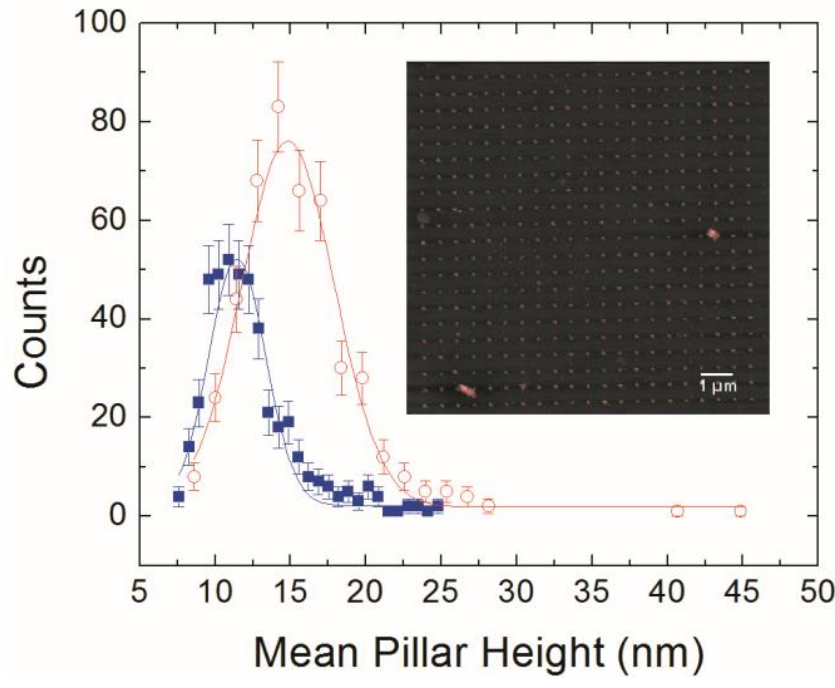

Figure 3.3 Height distributions for pillars before (blue squares) and after (red circles) CYP2C9 attachment. Fittings to a Gaussian model gives a peak center of $11.2 \mathrm{~nm}$ with a width of $3.7 \mathrm{~nm}$ for bare gold pillars (blue curve), and a peak center of $14.7 \mathrm{~nm}$ with a width of 5.9 $\mathrm{nm}$ after CYP2C9 attachment (red curve). Inset: AFM Height image of pillar array with SAM/CYP2C9 attached. Pillars (highlighted in red) were selected for height analysis through grain height thresholding. pillars with SAM/CYP2C9 attached can be seen in Figure 3.3. A shift in pillar height of $3.5 \mathrm{~nm}$ from bare gold pillars to CYP2C9 immobilized pillars can be easily observed in the data. In addition, the peak width increased from $3.7 \mathrm{~nm}$ for bare gold pillars to $6.7 \mathrm{~nm}$ for SAM/CYP2C9 attached pillars. The increase in pillar height with attached SAM/CYP2C9 is in good agreement with attachment of CYP2C9 to approximately $50 \%$ of our pillars given the proteins spherical diameter of $6 \mathrm{~nm}^{6}$. This is further validated by the increase seen in the peak width, which implies that, after attachment, an increase in heterogeneity is observed on our arrays. This is consistent with some pillars bearing attached protein and some remaining without bonded protein. Furthermore, the change in height and peak width is too small to support the case where multiple proteins would be stacking on top of one another, providing strong evidence that we are measuring ETp through a single P450 layer. 
CPAFM I-V curves provide conductance information which occurs through pathways known to dominate the ETp process ${ }^{252}$. Previously we observed that our gold nanopillars yield an ohmic response when voltage is applied, and that attachment of SAM leads to insulating behavior that is slightly reversed by bonding of CYP2C946. To obtain data for CYP2C9, at least five curves were averaged, all of them going from a maximum positive to a minimum negative voltage. The I-V curves measured as a function of applied force for P450 attached to a gold nanopillar are shown in Figure 3.4 for the enzyme alone and in the presence of substrate (flurbiprofen). In both cases, the current increased with probe force, as expected for an organic medium ${ }^{251,253}$. In contrast, no force dependent increase in current was observed for $I-V$ curves taken on the silicon surrounding the pillars.

Force dependent studies demonstrate that a reliable electrical contact to our protein is made at $16 \mathrm{nN}$. At $32 \mathrm{nN}$ the $I-V$ curves

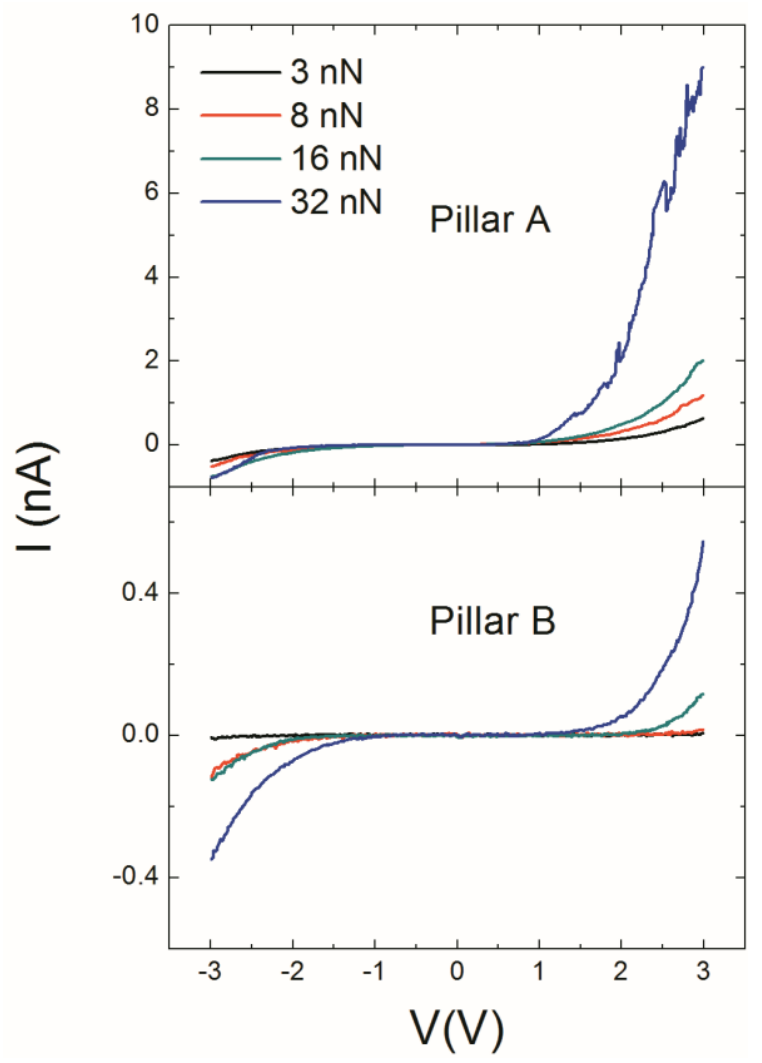

Figure 3.4. Measured I-V curves as a function of force for two nanopillars with attached CYP2C9. Pillar A contains CYP2C9 alone, Pillar B contains CYP2C9 with substrate flurbiprofen attached. Data obtained from two different samples.

indicate a significant loss in resistance, implying a large degree of compression ${ }^{254}$. Therefore, 16 $\mathrm{nN}$ was chosen as the applied force to obtain I-V data of CYP2C9 under various conditions. 
Figure 3.5 shows substrates/inhibitor used in our CPAFM studies of three different nanopillars whose $I-V$ data are graphed in Figure 3.6. All $I-V$ measurements listed for nanopillars were made<smiles>CC(C(=O)O)c1ccc(-c2ccccc2)c(F)c1</smiles><smiles>Nc1ccc(S(=O)(=O)c2ccc(N)cc2)cc1</smiles><smiles>Nc1ccc(S(=O)(=O)Nc2ccnn2-c2ccccc2)cc1</smiles>

Figure 3.5. Structures of the CYP2C9 substrates flurbiprofen and dapsone and the CYP2C9 inhibitor sulfaphenazole. on the same pillar and thus the same CYP2C9 enzyme and with the same applied tip force $(16 \mathrm{nN})$. To vary the substrate bound to the CYP2C9, the nanopillar was treated with substrate solutions under saturating conditions. Once a series of measurements were made, the substrate was washed out by immersion in phosphate buffer, and then immersed in a solution containing a different substrate at a saturating concentration in phosphate buffer.
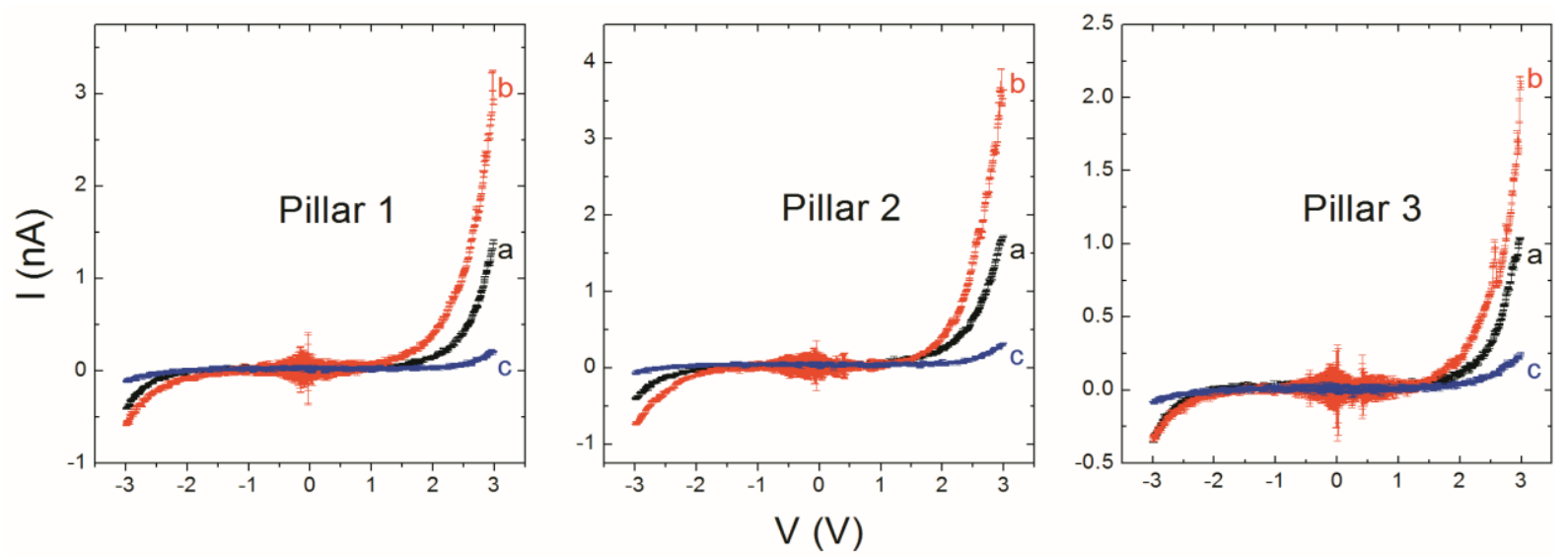

Figure 3.6. I-V curves measured for three nanopillars of (a) CYP2C9 alone, (b) CYP2C9 with flurbiprofen and dapsone, and (c) CYP2C9 with inhibitor sulfaphenazole.

Substrates that enter the active site in solution cannot escape once the sample is removed from solution and blown dry, and thus they are stuck in the active site during the in-air CPAFM measurements. We note that curves were consistent over sequential measurements after removal and re-insertion of samples (data not shown), and that the platform provided reproducible curves in the case of no substrate/inhibitor presence within the active site (Figure 3.7). 

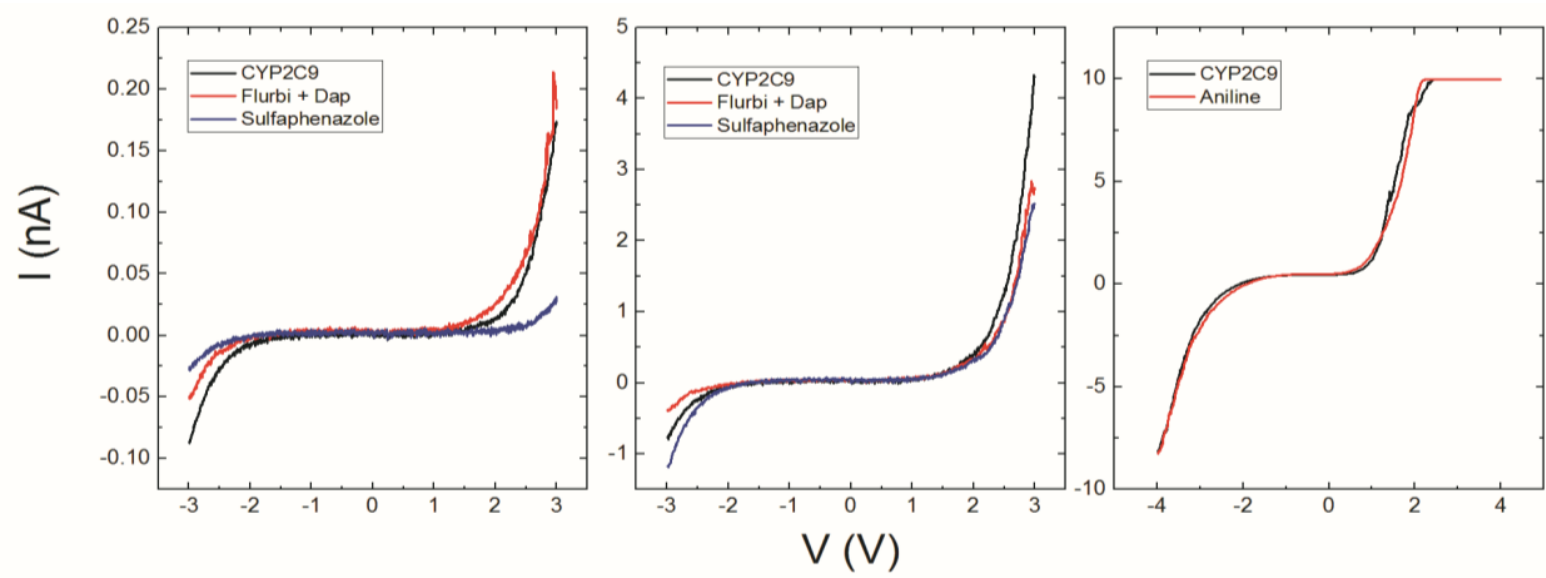

Figure 3.7. Measured I-V curves of three different nanopillars after different incubations with substrate/inhibitor. Measurements were taken in separate sessions up to 24 hours apart, and included removal of sample from AFM, followed by treatment with substrate/inhibitor, and finally re-insertion of sample into AFM and measurement. The left graph shows unchanging curves after treatment with substrate shown in studies to increase conductance, but shows reduced conductance with inhibitor. The middle graph shows unchanging I-V curves for all conditions, but demonstrates reproducibility of measurement. The right graph shows unchanging I-V curves denoting no entry by inhibitor aniline, and demonstrating reproducibility of measurement. These data exhibit the possible lack of substrate/inhibitor into the active site in some immobilized enzymes, and show strong reproducibility in I-V curves in successive measurements.

Here we compare CYP2C9, flurbiprofen/dapsone (metabolized by CYP2C9, see Figure 3.6) and sulfaphenazole (a CYP2C9 inhibitor, see Figure 3.6). First, I-V data were obtained on CYP2C9 by itself (Figure 3.6a). Next, the nanopillar array was immersed in a mixture of flurbiprofen and dapsone. Flurbiprofen and dapsone are substrates readily metabolized by CYP2C9. We have previously demonstrated that flurbiprofen and dapsone can occupy the active site simultaneously ${ }^{48}$. The $I-V$ data demonstrate that with both flurbiprofen and dapsone present with CYP2C9, the enzyme conductance is increased (Figure 3.6b), in good agreement with our previous findings of simultaneous binding of both substrates in the active site ${ }^{46}$. We have previously demonstrated that flurbiprofen metabolism is activated by the presence of dapsone ${ }^{50}$. Thus, the $I-V$ data showing increased conductance agree with the hypothesis that the rate of P450-mediated metabolism accelerates as ET becomes easier.

Following the $I-V$ measurements of the CYP2C9-flurbiprofen/dapsone system, the nanopillars were rinsed with water and treated with a solution of sulfaphenazole (Figure 3.6c) under saturating conditions. Sulfaphenazole is a known inhibitor of CYP2C9-mediated metabolism, that is 
purported to stabilize the heme iron in the low spin state ${ }^{45}$. The $I-V$ data for CYP2C9 with sulfaphenazole bound indicates that ETp is more difficult than the CYP2C9 alone or when readily metabolized substrates are bound (Figure 3.6c). This is in agreement with our previous data regarding aniline, another inhibitor of CYP2C9. Like sulfaphenazole, aniline is a Type II competitive inhibitor purported to stabilize the heme iron in the low spin state ${ }^{45}$, and thus when bound to CYP2C9, it reduced the ease of ET. We have previously demonstrated that the ease of P450 reduction and spin state are not well correlated, as CYP2C9 complexes with either flurbiprofen or dapsone had identical conductance profiles ${ }^{46}$, despite flurbiprofen being known to stabilize the high spin state, and dapsone having little effect on spin state. In addition, ETp was much easier for dapsone than aniline, despite neither altering spin state. Our results with sulfaphenazole indicate that inhibitors may also block ETp. This result presented herein is now the second example of this type of behavior. It is also a novel result because P450 inhibitors are thought to act only by binding to the active site and blocking access to it, and/or stabilizing the heme iron in the low spin state. Finally, we note that sulfaphenazole was chosen due to its similar inhibitory properties in comparison to aniline. However, sulfaphenazole binds tightly to CYP2C9 while aniline binds only weakly ${ }^{45}$. Therefore, the strength of binding does not seem to affect the inhibitor's ability to block ET.

A quantitative analysis was undertaken to better understand the information embedded in the I- $V$ curves. While the Simmons model is generally used for CPAFM analysis at low bias ${ }^{253,254}$, this was not a suitable model for this system due to the low conductivity near $V_{B}=0$. Measurements of conductivity near bias voltage $V_{B}=0$ were zero, and remained zero (to within the noise floor of the data, $\pm 3 \mathrm{pA}$ ) until the conductance turned on at a critical voltage (see Figure 3.6). This indicates that the tunneling barrier for the electrons transferred from the gold nanopillar to the CPAFM tip was large in energy. Instead, the data were fit with the Poole-Frenkel (PF) emission model $^{255}$ to compare with our previous findings and look for reproducible trends. In the PF emission model electrons are trapped in localized states and conduct by hopping from one 
localized state to another within an insulating layer. This model is appropriate for our system in which the protein-SAM, the insulator, and various functional groups within the protein serve as areas where localized states may occur and trap electrons between them ${ }^{6,24,152,158}$.

In the PF model, the current to voltage ratio $I / V$ is expected to behave as

$$
\ln (I / V)=\ln C-\frac{q \Phi_{B}}{k T}+\frac{q}{k T}\left(\frac{q}{\pi d \epsilon_{0} \epsilon_{S}}\right)^{1 / 2} V^{1 / 2},
$$

where $V$ is the applied (bias) voltage, $q$ is the charge of an electron, $\Phi_{B}$ is the effective voltage barrier that the electron must overcome to move from one localized state to another, $d$ is the distance across which the voltage is applied, $\varepsilon_{0}$ is the permittivity of free space, and $\varepsilon_{s}$ is the relative permeability of the material (in this case CYP2C9 and SAM) at high frequencies, assuming that there is no local polarization induced. Also, $k$ is the Boltzmann's constant, $T$ is the absolute temperature, $C$ is a constant that depends on the intrinsic mobility of the charge carriers, the effective area of the electrical contact, and the effective distance $d$ across which $V$ is applied. A plot of $\ln (I / V)$ as a function of $V^{1 / 2}$ should therefore yield a straight line with the $y$-intercept proportional to $\Phi_{B}$. The slope should be sensitive to changes in the effective size $(d)$ of the protein and its effective dielectric constant. As shown previously ${ }^{46}$, although it is not possible to solve for $\Phi_{B}$ because the value of $C$ is unknown, it is possible to obtain relative changes in $d$ and $\Phi_{B}$ assuming that $C$ remains unchanged upon binding of different substrates. 
Figure 3.8 contains a plot of $\ln (\mathrm{I} / \mathrm{V})$ as a function of $V^{1 / 2}$ for the positive bias data for the nanopillars shown in Figure 3.6. The data were fit to Equation 1 for $V>1 \mathrm{~V}$. Data from smaller voltages were poorly fit by the model as was observed in our previous work ${ }^{46}$, and it is possible another mechanism is at play in this region. The quantitative results are presented in Table 3.1, where $\Delta \Phi_{B}$ is the shift of barrier height from the run in which there was no substrate bound in the active site of CYP2C9. The fractional change $\Delta d / d_{0}$, where $d_{0}$ is the distance between hopping sites, was also calculated. The data in Table 3.1 reveal that the slopes for all conditions were similar, implying that $d$ and $\varepsilon_{s}$ were similar in all runs as hypothesized. Thus observed differences in the $y$-intercept should be due to changes seen in $\Phi_{B}$.
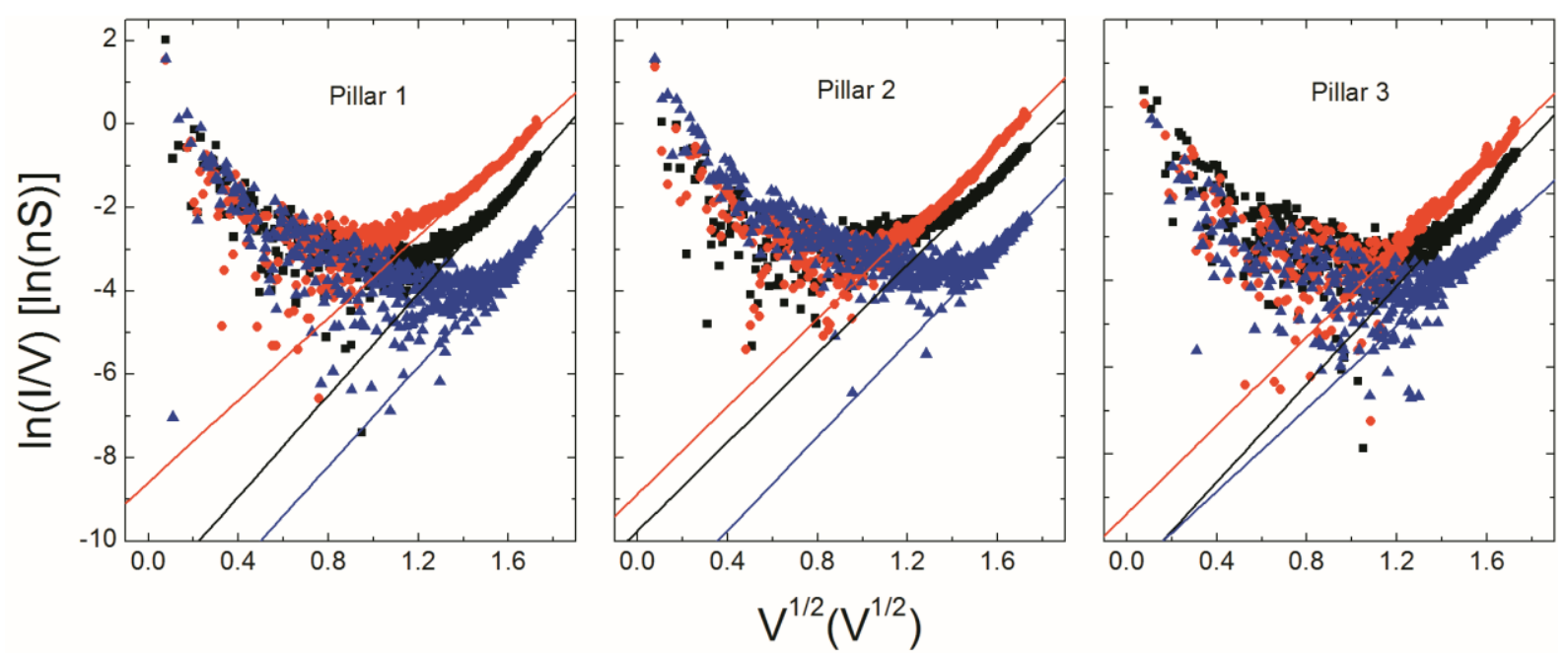

Figure 3.8. Poole-Frenkel plot for the data obtained for positive bias voltages for nanopillars in Figure 3.6. CYP2C9 alone (black), CYP2C9 with flurbiprofen and dapsone (red), and CYP2C9 with inhibitor sulfaphenazole (blue). The symbols are the data and the lines are fits to a straight line. 
Table 3.1 shows that for all nanopillars, the addition of flurbiprofen and dapsone decreased the barrier height. In contrast, the addition of sulfaphenazole does not decrease the barrier height,

Table 3.1 Results of the Fits to the I-V data with the Poole-Frenkel model. ${ }^{a}$

\begin{tabular}{|l|l|l|l|}
\hline & CYP2C9 & Flurbi+Dap & Sulfaphenazole \\
\hline Pillar 1 & & & \\
\hline Intercept & -11.38 & -8.59 & -12.97 \\
\hline Slope & 6.10 & 4.92 & 5.96 \\
\hline$\Delta \phi$ B & 0 & -0.07 & 0.04 \\
\hline$\Delta \mathrm{d} / \mathrm{d}_{0}$ & 0 & 0.39 & 0.04 \\
\hline Pillar 2 & & & \\
\hline Intercept & -9.76 & -8.88 & -12.01 \\
\hline Slope & 5.32 & 5.25 & 5.65 \\
\hline$\Delta \Phi_{\mathrm{B}}$ & 0 & -0.02 & 0.06 \\
\hline$\Delta \mathrm{d} / \mathrm{d}_{0}$ & 0 & 0.03 & -0.12 \\
\hline Pillar 3 & & & \\
\hline Intercept & -10.90 & -9.40 & -10.77 \\
\hline Slope & 5.64 & 5.09 & 4.77 \\
\hline$\Delta \Phi_{\mathrm{B}}$ & 0 & -0.04 & -0.003 \\
\hline$\Delta \mathrm{d} / \mathrm{d}_{0}$ & 0 & 0.19 & 0.31 \\
\hline
\end{tabular}

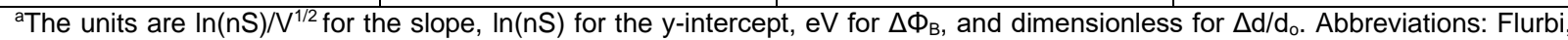
flurbiprofen; Dap, dapsone. ${ }^{\mathrm{b}} \mathrm{A}$ value of $\mathrm{T}=300 \mathrm{k}$ was used to calculate $\Delta \Phi_{\mathrm{B}}$. The error for intercept and slope values were $<4 \%$.

and might slightly increase it. The general trend for the width of the barrier is that of an increase in the addition of substrate, except for sulfaphenazole in nanopillar 2 . These trends are consistent with the general properties of CYP2C9 and how it interacts with these particular substrates. The reduction of CYP2C9 is easier in the presence of metabolized substrates (flurbiprofen and dapsone), but more difficult in the presence of inhibitor (sulfaphenazole). 
Figure 3.9 depicts a plot of $\ln (I / V)$ as a function of $V^{1 / 2}$ for the positive bias data for the nanopillars presented in Figure 3.4. The data were fit to Equation 1 for $V>1 \mathrm{~V}$ as was done in Figure 3.8. For the enzyme alone (pillar A), measured with the largest force $(32 \mathrm{nN})$, the plot reveals a possible phase transition as seen by the multiple linear regions. We fit all three possible linear regions to avoid biasing the data (Figure 3.10). The lack of phase transition observed in the

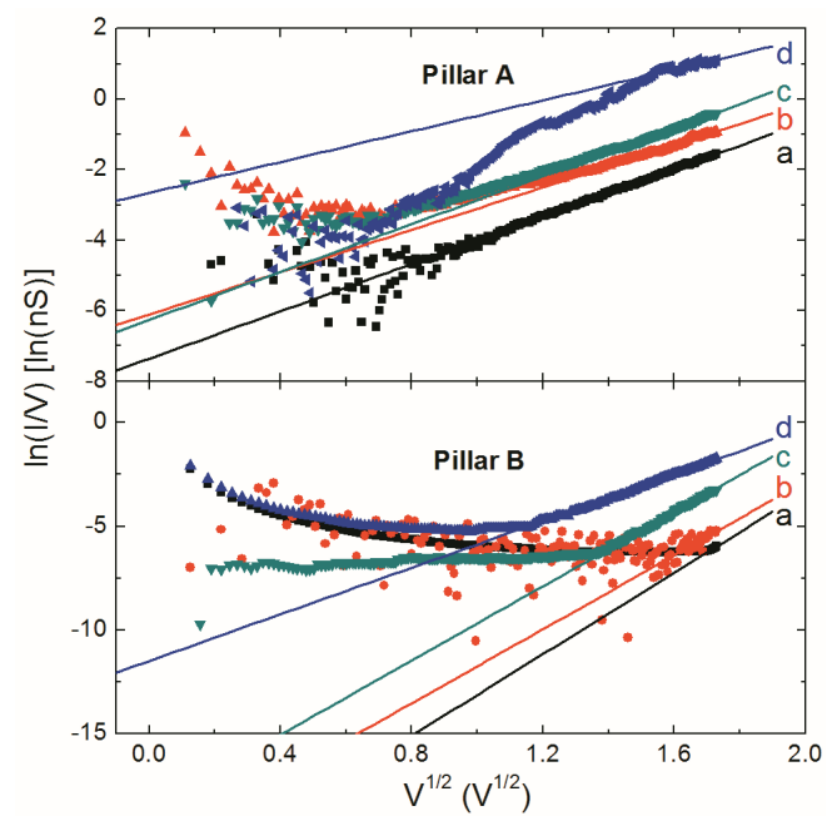

Figure 3.9. Poole-Frenkel plot for the data obtained for positive bias voltages for nanopillars in Figure 3.4 using same color code. Force was modulated with measurements (a) at 2-3 nN, (b) at $8 \mathrm{nN}$, (c) at $16 \mathrm{nN}$, and (d) at $32 \mathrm{nN}$. The symbols are the data and the lines are fits to a straight line. $32 \mathrm{nN}$ on Pillar $A$ was fit for all three linear regimes, with Fit 1 between 1.6 to $1.8 \mathrm{~V}^{1 / 2}$, Fit 2 between 1.2 to $1.6 \mathrm{~V}^{1 / 2}$, and Fit 3 between 1 to $1.2 \mathrm{~V}^{1 / 2}$ (see Figure 3.10).

presence of flurbiprofen could be explained by the known stabilizing effect of substrate binding within $\mathrm{P} 450 \mathrm{~s}^{256}$. The quantitative results are plotted in Figure 3.11 with the same description of variables found in Table 3.1. The relatively large error bars for the Pillar B at $8 \mathrm{nN}$ data are due to a limited linear regime to fit the PF model (see Figure 3.9). Using the low force $(2-3 \mathrm{nN})$ data as the baseline, a decrease in the barrier height with increased applied force (both pillars) is observed. Fit 1 for Pillar A at $32 \mathrm{nN}$ is in good agreement with the trend seen in Pillar B. Fit 2 and 3 both result in an increase in barrier height, which disagrees with the general trend that a decrease in barrier height is associated with an increased applied force ${ }^{253}$. We thus believe that Fit 1 is the correct region to analyze. 
In both pillars there is no change in slope until the highest force is applied (32 $\mathrm{nN})$. This difference in slope implies a change in $d$ or $\varepsilon_{s}$, in contrast to our substrate/inhibitor analysis conducted at a single force, where only minimal changes in slope were observed. Using Fit 1 again for Pillar A at $32 \mathrm{nN}$, good agreement with Pillar B is obtained for $\Delta d / d_{0}$ values.

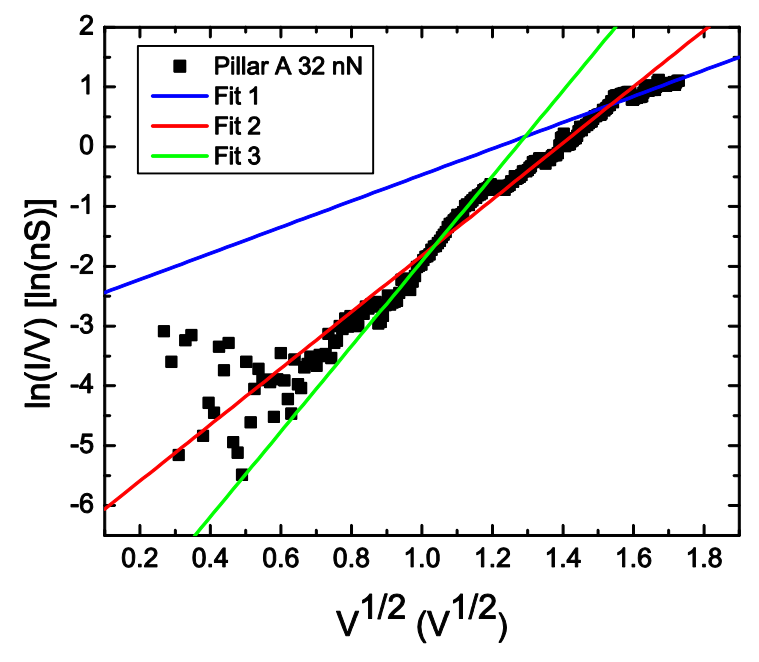

Figure 3.10. Poole-Frenkel plot for I-V curves taken at 32 $n N$ on Pillar A. All three linear regions were fit to obtain values of barrier height and barrier width in Figure 3.11.

Under increased force we expect the protein to become compressed 253,254 . Compression of proteins has also been shown to correlate with a decrease in barrier height ${ }^{257}$, which agrees with our results in Figure 3.11a. Interestingly, Figure 3.11b shows an increase in the spacing of localized states with increased force. This would indicate that even though the P450 is being compressed, the spacing between localized states within the P450 increases on average. 


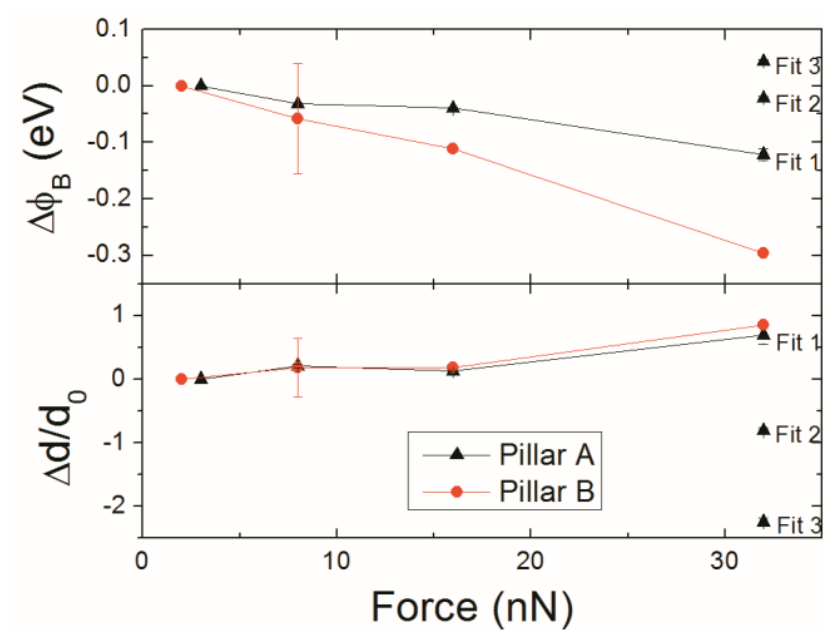

Figure 3.11 a) Change in barrier height as a function of force. Lowest force used as the baseline to generate barrier height values. b) Change in $d / d$ o as a function of force with lowest force used as a baseline to determine values. Data calculated using PooleFrenkel as done in Table 3.1.

These results can be explained by a zig-zag path of electron flow through the protein's different motifs between localized that are not aligned the vertical direction, as shown in Figure 3.12. This can be demonstrated by noting that the distance between two hopping sites is $d=\sqrt{a^{2}+c^{2}}$, where $a$ is the in-plane component and $c$ is the vertical component of the vector $\mathbf{d}$ which extends from one state to the other. Small changes in a and $c$ would result in a small change in $d$ with respect to the change in $c$ of

$$
\frac{\delta d}{\delta c}=\frac{c}{d}+\frac{a}{d} \frac{\delta a}{\delta c}
$$

We now assume that the volume of the protein that contains the two hopping sites is proportional to $V \propto a^{2} c$, and that this volume remains constant when the protein is compressed, that is, the protein deforms when it is pressed by the tip from above. Mathematically, this means that the change in volume

$$
\delta V \propto 2 a c \delta a+a^{2} \delta c=0
$$


Solving equation 4 to obtain a relation between $\delta a$ and $\delta c$, and combining this result with equation 3 , yields a change of the total distance between hopping sites with respect to the change in vertical distance of

$$
\frac{\delta d}{\delta c}=\frac{2 c^{2}-a^{2}}{2 d a}
$$
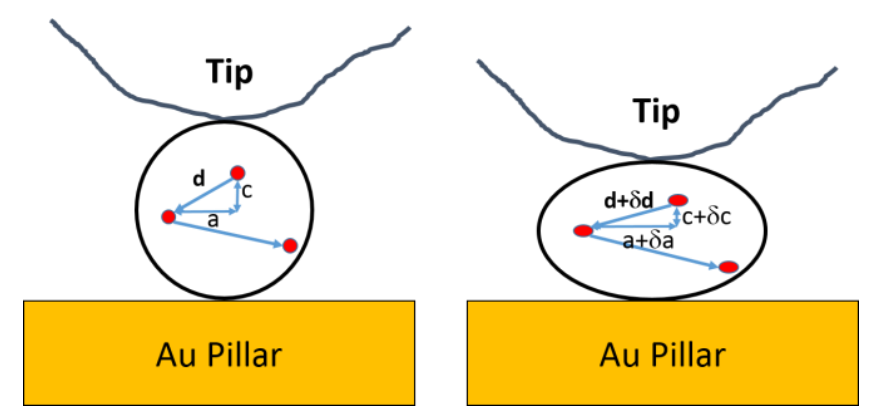

Figure 3.12 Simplified depiction of electron transfer through hopping sites (red circles) within a protein immobilized to gold under non-compressed (left) and compressed (right) conditions. The vector $\boldsymbol{d}$ determines the relative position between the top two hopping sites and $a$ and $c$ indicate the horizontal and vertical distance components, respectively. The values of $\delta \boldsymbol{d}$, $\delta a$ and $\delta c$ are the changes in the vector and distance components when the protein is compressed by the tip.

Equation 5 shows that decreasing the vertical distance $(\delta c<0)$ leads to either an increase or decrease is $d$ depending on whether $2 c^{2}-a^{2}$ is negative or positive, respectively. Our data seem to indicate that, on average, the hopping sites are located such that $a>\sqrt{2} c$. Currently there is no way to determine which specific functional groups in the protein serve as these impurities in order to model their positions under compression by the AFM tip, and further work is necessary to elucidate this process. In any case, the force data further validate that our choice of $16 \mathrm{nN}$ is appropriate and does not lead to large changes in our enzyme size due to CPAFM tip interaction.

\subsection{Conclusions}

We have developed an effective platform to measure the conductance of immobilized proteins that eliminates aggregation effects on ET studies. The data demonstrate that for immobilized CYP2C9 proteins, a correlation exists between the energy barrier height between hopping sites, $\Phi_{B}$, and CYP2C9 metabolic activity. An inverse correlation between $\Phi_{B}$ and tip force was also observed which agrees with other prior work. Interestingly, the data indicate that the protein is 
more stable in the presence of a substrate when subjected to a high tip force. This agrees with the substrate entry into the active site helping to stabilize enzyme conformation. For large forces, the relative distance between hopping sites $\Delta d / d_{0}$ also increases, possibly because protein secondary structures such as alpha helices and beta sheets often serve as pathways for ET ${ }^{127}$, and thus there are numerous ways in which electrons could travel through P450s to obtain this change in $\Delta d / d_{o}$.

Another inhibitor (sulfaphenazole), in addition to the previously studied aniline, was found to increase the barrier height for ETp and thereby makes CYP2C9 reduction more difficult and inhibits metabolism. Although P450 inhibitors are thought to compete for binding to the active site and not be metabolized ${ }^{258}$, our findings suggest that P450 Type II ligands may also decrease the ease of ET processes in the enzyme. Overall we show that our nanopillar platform allows for repetitive and reproducible studies of the same unmodified immobilized molecule in sequential studies. Future studies can be undertaken with this platform to identify protein-protein effects using hetero or homo-dimers ${ }^{259}$, as well as substrate motifs important for metabolism using substrate analogs ${ }^{260}$. 
Chapter 4

\section{Summary}




\subsection{Summary}

In this work the effectors of P450 mediated metabolism were studied using an immobilized platform to control P450 aggregation. Controlled immobilization of P450s to a SAM on gold allows for an enzymatically active P450 that mimics its in vivo conformation. Utilizing this methodology, the nature of P450-P450 interactions was investigated in Chapter 2. CYP2C9 was immobilized to gold via SAM and hetero/homomeric complexes of CYP2C9-CYP2C9, CYP2C9-CYP2D6, CYP2C9-CYP3A4, and CYP2C9-CPR were evaluated. SPR binding analysis revealed potent binding between all of the aforementioned complexes, and demonstrated P450-P450 complexes formed more readily than P450-CPR interactions in the case of CYP2C9-CYP2D6 and CYP2C9CYP2C9.

Using this same immobilization scheme, the effect of P450-P450 interaction on metabolism was evaluated, and compared to the standard solution based reconstituted system with nonimmobilized protein. Given that P450s are membrane bound in vivo, it is likely the amount of aggregation observed in solution based reconstituted systems is not indicative of the in vivo environment. This is of great importance for predicting in vivo metabolism from in vitro data, as it has been well documented that P450 interactions can alter P450 catalytic rates. Studies revealed that metabolic outcomes resulting from P450-P450 interactions were isoform specific, and resulted in both activation and inhibition of CYP2C9 mediated metabolism. It is also demonstrated, by order of addition studies, that the mechanism causing these effects is isoform specific.

Lastly, this study demonstrated, for the first time, the homomeric activation of CYP2C9 mediated metabolism. The controlled immobilization of enzymatically active CYP2C9 confers the unique ability to measure homo-dimerization effects on metabolism, shedding light on possible P450 mechanisms. This is made possible due to hindered metabolism of lipidless P450s in solution. We also demonstrate differences in the effect of P450-P450 interactions in our 
immobilized platform compared to a solution based reconstituted system. We observe strong activation of CYP2C9 metabolism in the presence of CYP2D6 in the solution based assay, but inhibition when CYP2C9 is immobilized. Given that the magnitude of the effects were equivalent at similar CYP2C9:CYP2D6 ratios, we can surmise that the differences in effect are correlated to the different binding modes available between the P450 binding partners. This result signifies the importance of understanding the mechanism of P450 interactions in drug metabolism predictions as our linked model is likely the more correct interpretation of the same parameters.

We further developed an understanding of these P450 interactions using molecular modeling to assess possible binding modalities. Our molecular modeling predictions are in good agreement with our in vitro SPR results, demonstrating the ability to predict possible P450 interactions in silico. P450 binding interactions were shown to form stable complexes that rarely involved the CPR binding site. Thus it is likely that P450 interaction influences on metabolism are due to resultant conformational changes altering P450 binding to CPR or substrate, rather than direct competition for the CPR binding site.

In Chapter 3, a nanopillar platform was employed to further control P450 aggregation. The fabrication of nanopillars of small radius $(20-40 \mathrm{~nm})$ and the use of a SAM allow isolation of a single or small group of immobilized CYP2C9. We demonstrate through height analysis that immobilization of our enzyme produces a single layer of CYP2C9 on top of the pillar and note that changes in height show approximately $50 \%$ of pillars containing CYP2C9, in good agreement with previous estimates of CYP2C9 coverage on gold films in our laboratory. These results demonstrated that our measurements were on a single P450 vertically, and only lateral interactions are possible. Given this, are ETp results would be on a single molecule as the easiest path for the current would be through a single protein.

We also demonstrated force dependent conductance in CYP2C9 using CPAFM. Our results indicated that CYP2C9 experiences compression at higher forces leading to enhanced 
conductance. This agrees with literature theory and results of compression of organic layers leading to enhanced conduction due to shorter electron transfer path. We also observed a difference in the stability of CYP2C9 conductance under force in the presence and absence of flurbiprofen. This demonstrated our platforms ability to measure the known enhanced stability of P450 upon substrate binding. We observed an increase in the distance of ETp under higher force, which is easily explained by a zig zag ETp path that is extended with P450 compression (Figure 3.12).

This platform was also indexed to allow study of the same molecule of CYP2C9 upon different substrate exposure. Given the importance of ET in P450 mediated metabolism, we investigated how ETp was influenced by the presence of different substrates and inhibitors with known metabolic profiles. Flurbiprofen and dapsone were chosen for investigation as we previously showed increases in CYP2C9 ETp due to simultaneous occupancy of both substrates within the active site. Our previous study also demonstrated the novel finding of aniline lowering ETp, indicating a possible secondary mechanism of CYP2C9 inhibition. In this report we demonstrated that sulfaphenazole, another competitive inhibitor like aniline, also decreased ETp through CYP2C9. This finding could be of interest as to why some molecules are not metabolized by P450s based on structure motifs, and could be implemented in better small molecule drug design. The ability to study immobilized proteins on nanopillars offers reduced variability in repeated studies, giving more precise details when measured.

\subsection{Future Directions}

Future studies on this immobilized platform could provide answers to questions about P450 interactions that have thus far eluded researchers. P450-P450 interactions modulate metabolism by either competing for CPR, altering CPR affinity, or altering substrate turnover. Thus it would be of interest to further explore how dimerization modulates CPR and substrate binding. An initial step would be to take the dimer complexes created in Chapter 2, and model the stability of 
substrate in the active site, in comparison to being in the active site of the monomeric P450. This could show how P450 complex formation may shift the position of the substrate in the active site and reveal mechanisms by which substrate turnover can be affected.

Another study could also be conducted on CPR affinity for a P450 complex. CPR could be blind docked utilizing GRAMMX to the dimer complexes obtained in Chapter 2 to reveal favorability of binding compared to CPR binding to monomeric P450s. This study could then be expanded in vitro by using homo/heteromeric fusion proteins to obtain binding constants via SPR. SPR also has the ability to measure kinetic binding constants for two different analytes simultaneously, which would allow us to measure, in real time, how CPR binding to an immobilized P450 changes in the presence of free competing P450. These studies would give us a clear understanding of how P450 interactions modulate CPR ET, and allow for us to better incorporate these effects into in vitro predictions of in vivo metabolism.

We obtained novel results for our CPAFM study, but to further investigate P450 ETp it is important that we understand the orientation of the P450. To figure out which lysines are bonded, we could conduct a proteomic study digesting bonded CYP2C9 and analyzing with mass spectrometry. When comparing enzyme fragments obtained from P450s not attached to the SAM, to those digested after immobilization to gold, we could determine which lysines are bonded by looking for an increase in molecular weight due to the attachment of MUA. This would give us the exact orientation of P450 in our immobilization scheme, and would allow studies such as the modeling of electron flow from the gold surface through the SAM and to the P450 heme group.

Another interesting study would be to compare ETp between mutant forms of P450s with that of normal P450 forms. For example, a mutation in the CYP2C9 gene causes the expression of three allelic variants (CYP2C9*1, CYP2C9*2, and CYP2C9*3). Both CYP2C9*2 and CYP2C9*3 have been shown to have altered kinetics for metabolism of warfarin a CYP2C9 substrate. We could use our knowledge of the rate of metabolism of these substrates to determine ETp 
correlation in CPAFM measurements. From our current studies we would expect to see a more conductive ETp profile for allelic variants that metabolize warfarin at a faster rate. This could enhance our understanding of why these single nucleotide polymorphisms cause such drastic changes in substrate metabolic profile. In addition, conducting CPAFM with immobilized fusion proteins of homodimers or heterodimers could show if modulation of ET plays a role in P450-P450 interactions. We could probe ET on these fusion proteins alone and in the presence of the substrates mentioned above to interrogate effects on ET, and whether they result from protein interactions, substrate interactions, or a combination of both.

In contrast to the CPAFM results presented in Chapter 3, ETp measurements conducted in solution using a closed cell would allow study of the dynamic environment that would closer model the physiological process where the substrate is not tightly bound in the active site, and can also be metabolized completely by the addition of electrons. Utilizing EC-STM, we could obtain mechanistic data about ET in immobilized P450s, and observe changes in heme reduction and conformation. EC-STM would also provide us with $\mathrm{E}^{\circ}$ value, a direct measure of the ease of ET. $\mathrm{E}^{\circ}$ values obtained for the enzyme alone could be compared with the values obtained upon administration of substrates. The high resolution of EC-STM would allow for single protein evaluation of substrate effects on the ease of heme reduction.

In order for ET to occur from CPR favorably there needs to be an anodic shift in the redox potential of P450. Thus if we see an anodic shift in $\mathrm{E}^{\circ}$ due to the presence of substrate we can infer that it is creating a more favorable situation for ET, and thus making itself easier for P450 metabolism. The choice of our substrates will show what factors are correlated with ㅌo changes. Lastly, ECSTM would provide a study without force modulation of the protein through tip contact that we could obtain I-V curves to compare with the CPAFM studies in Chapter 3 to observe how conformational change due to tip interaction alters ET. This would enhance our understanding of our obtained results. 


\subsection{Conclusion}

It has been well documented that solution based P450 assays offer poor control of P450 aggregation. We demonstrate that our immobilized P450 platform allows study of a metabolically active monomeric enzyme. This allows interrogation of ETp, protein-protein interactions, substrate-protein interactions, and atypical enzyme kinetics with a P450 attachment that mimics the in vivo environment. Ability to control aggregation of P450s allows enhanced understanding of metabolic effectors, and may help improve in vivo substrate metabolism predictions in vitro. 
Chapter 5

References 


\section{References}

1. Mueller, G. C. \& Miller, J. A. The Metabolism of 4-Dimethylaminoazobenzene by Rat Liver Homogenates. J. Biol. Chem. 176, 535-544 (1948).

2. Omura, T. \& Sato, R. A New Cytochrome in Liver Microsomes. J. Biol. Chem. 237, PC1375-PC1376 (1962).

3. Lu, A. Y. H. \& Coon, M. J. Role of Hemoprotein P-450 in Fatty Acid w-Hydroxylation in a Soluble Enzyme System from Liver Microsomes. J. Biol. Chem. 243, 1331-1332 (1968).

4. Distlerath, L. M. et al. Purification and characterization of the human liver cytochromes P450 involved in debrisoquine 4-hydroxylation and phenacetin O-deethylation, two prototypes for genetic polymorphism in oxidative drug metabolism. J. Biol. Chem. 260, 9057-9067 (1985).

5. Barnes, H. J., Arlotto, M. P. \& Waterman, M. R. Expression and enzymatic activity of recombinant cytochrome P450 17 alpha-hydroxylase in Escherichia coli. Proc. Natl. Acad. Sci. U. S. A. 88, 5597-5601 (1991).

6. Wester, M. R. et al. The Structure of Human Cytochrome P450 2C9 Complexed with Flurbiprofen at 2.0-Å Resolution. J. Biol. Chem. 279, 35630-35637 (2004).

7. Sevrioukova, I. F., Li, H., Zhang, H., Peterson, J. A. \& Poulos, T. L. Structure of a cytochrome P450-redox partner electron-transfer complex. Proc. Natl. Acad. Sci. U. S. A. 96, 1863-1868 (1999).

8. Nelson, D. R. et al. The P450 Superfamily: Update on New Sequences, Gene Mapping, Accession Numbers, Early Trivial Names of Enzymes, and Nomenclature. DNA Cell Biol. 12, 1-51 (1993).

9. Nebert, D. W. et al. The P450 gene superfamily: recommended nomenclature. DNA Mary Ann Liebert Inc 6, 1-11 (1987). 
10. Steward, D. J. et al. Genetic association between sensitivity to warfarin and expression of CYP2C9*3. Pharmacogenetics 7, 361-367 (1997).

11. Szczesna-Skorupa, E. \& Kemper, B. An N-terminal glycosylation signal on cytochrome P450 is restricted to the endoplasmic reticulum in a luminal orientation. J. Biol. Chem. 268, 1757-1762 (1993).

12. Miller, W. L. Minireview: Regulation of Steroidogenesis by Electron Transfer. Endocrinology 146, 2544-2550 (2005).

13. Guengerich, F. P. Cytochrome P450s and other enzymes in drug metabolism and toxicity. AAPS J. 8, E101-E111 (2006).

14. Williams, J. A. et al. Drug-Drug Interactions for Udp-Glucuronosyltransferase Substrates: A Pharmacokinetic Explanation for Typically Observed Low Exposure (auci/Auc) Ratios. Drug Metab. Dispos. 32, 1201-1208 (2004).

15. Guengerich, F. P. Mechanisms of cytochrome P450 substrate oxidation: MiniReview. J. Biochem. Mol. Toxicol. 21, 163-168 (2007).

16. Guengerich, F. P. \& Johnson, W. W. Kinetics of Ferric Cytochrome P450 Reduction by NADPH-Cytochrome P450 Reductase: Rapid Reduction in the Absence of Substrate and Variations among Cytochrome P450 Systems†. Biochemistry (Mosc.) 36, 14741-14750 (1997).

17. Locuson, C. W., Gannett, P. M. \& Tracy, T. S. Heteroactivator effects on the coupling and spin state equilibrium of CYP2C9. Arch. Biochem. Biophys. 449, 115-129 (2006).

18. Yamazaki, H. et al. Reconstitution of Recombinant Cytochrome P450 2C10(2C9) and Comparison with Cytochrome P450 3A4 and Other Forms: Effects of Cytochrome P450P450 and Cytochrome P450-b5Interactions. Arch. Biochem. Biophys. 342, 329-337 (1997). 
19. Shimada, T., Mernaugh, R. L. \& Guengerich, F. P. Interactions of mammalian cytochrome P450, NADPH-cytochrome P450 reductase, and cytochrome b5 enzymes. Arch. Biochem. Biophys. 435, 207-216 (2005).

20. Wang, M. et al. Three-dimensional structure of NADPH-cytochrome P450 reductase: Prototype for FMN- and FAD-containing enzymes. Proc. Natl. Acad. Sci. U. S. A. 94, 8411-8416 (1997).

21. Iyanagi, T., Xia, C. \& Kim, J.-J. P. NADPH-cytochrome P450 oxidoreductase: prototypic member of the diflavin reductase family. Arch. Biochem. Biophys. 528, 72-89 (2012).

22. Bridges, A. et al. Identification of the Binding Site on Cytochrome P450 2B4 for Cytochrome b 5 and Cytochrome P450 Reductase. J. Biol. Chem. 273, 17036-17049 (1998).

23. Gutierrez, A. et al. Electron transfer in human cytochrome P450 reductase. Biochem. Soc. Trans. 31, 497-501 (2003).

24. Pudney, C. R., Khara, B., Johannissen, L. O. \& Scrutton, N. S. Coupled Motions Direct Electrons along Human Microsomal P450 Chains. PLoS Biol. 9, (2011).

25. Estabrook, R. W., Franklin, M. R., Cohen, B., Shigamatzu, A. \& Hildebrandt, A. G. Biochemical and genetic factors influencing drug metabolism. Influence of hepatic microsomal mixed function oxidation reactions on cellular metabolic control. Metabolism. 20, 187-199 (1971).

26. Reed, J. R., Cawley, G. F. \& Backes, W. L. Inhibition of Cytochrome P450 1A2-Mediated Metabolism and Production of Reactive Oxygen Species by Heme Oxygenase-1 in Rat Liver Microsomes. Drug Metab. Lett. 5, 6-16 (2011).

27. Kawato, S., Gut, J., Cherry, R. J., Winterhalter, K. H. \& Richter, C. Rotation of cytochrome P-450. I. Investigations of protein-protein interactions of cytochrome P-450 in phospholipid vesicles and liver microsomes. J. Biol. Chem. 257, 7023-7029 (1982). 
28. Gut, J., Richter, C., Cherry, R. J., Winterhalter, K. H. \& Kawato, S. Rotation of cytochrome P-450. Complex formation of cytochrome P-450 with NADPH-cytochrome P-450 reductase in liposomes demonstrated by combining protein rotation with antibody-induced crosslinking. J. Biol. Chem. 258, 8588-8594 (1983).

29. Peterson, J. A., Ebel, R. E., O'Keeffe, D. H., Matsubara, T. \& Estabrook, R. W. Temperature dependence of cytochrome P-450 reduction. A model for NADPHcytochrome P-450 reductase:cytochrome P-450 interaction. J. Biol. Chem. 251, 40104016 (1976).

30. Eyer, C. S. \& Backes, W. L. Relationship between the rate of reductase-cytochrome P450 complex formation and the rate of first electron transfer. Arch. Biochem. Biophys. 293, 231-240 (1992).

31. Taniguchi, H., Imai, Y., lyanagi, T. \& Sato, R. Interaction between NADPH-cytochrome P450 reductase and cytochrome P-450 in the membrane of phosphatidylcholine vesicles. Biochim. Biophys. Acta 550, 341-356 (1979).

32. Guengerich, F. P. Common and Uncommon Cytochrome P450 Reactions Related to Metabolism and Chemical Toxicity. Chem. Res. Toxicol. 14, 611-650 (2001).

33. Meunier, B., de Visser, S. P. \& Shaik, S. Mechanism of Oxidation Reactions Catalyzed by Cytochrome P450 Enzymes. Chem. Rev. 104, 3947-3980 (2004).

34. Manyike, P. T., Kharasch, E. D., Kalhorn, T. F. \& Slattery, J. T. Contribution of CYP2E1 and CYP3A to acetaminophen reactive metabolite formation. Clin. Pharmacol. Ther. 67, 275-282 (2000).

35. Cameron, R., Feuer, G. \& de la Iglesia, F. Drug-Induced Hepatotoxicity. (Springer Science \& Business Media, 2012).

36. Cuttle, L. et al. Phenytoin Metabolism by Human Cytochrome P450: Involvement of P450 3A and 2C Forms in Secondary Metabolism and Drug-Protein Adduct Formation. Drug Metab. Dispos. 28, 945-950 (2000). 
37. Arlt, V. M. et al. 3-Aminobenzanthrone, a Human Metabolite of the Environmental Pollutant 3-Nitrobenzanthrone, Forms DNA Adducts after Metabolic Activation by Human and Rat Liver Microsomes: Evidence for Activation by Cytochrome P450 1A1 and P450 1A2. Chem. Res. Toxicol. 17, 1092-1101 (2004).

38. Haouzi, D. et al. Cytochrome P450-generated reactive metabolites cause mitochondrial permeability transition, caspase activation, and apoptosis in rat hepatocytes. Hepatol. Baltim. Md 32, 303-311 (2000).

39. Isley, W. L. \& Oki, J. C. Hepatotoxicity of thiazolidinediones. Diabetes Obes. Metab. 3, 389-392 (2001).

40. Shimada, T. et al. Metabolism of Benzo[a]pyrene to trans-7,8-Dihydroxy-7,8dihydrobenzo[a]pyrene by Recombinant Human Cytochrome P450 1B1 and Purified Liver Epoxide Hydrolase. Chem. Res. Toxicol. 12, 623-629 (1999).

41. Sanderson, J. P., Naisbitt, D. J. \& Park, B. K. Role of bioactivation in drug-induced hypersensitivity reactions. AAPS J. 8, E55-E64 (2006).

42. Baer, B. R., Wienkers, L. C. \& Rock, D. A. Time-dependent inactivation of P450 3A4 by raloxifene: identification of Cys239 as the site of apoprotein alkylation. Chem. Res. Toxicol. 20, 954-964 (2007).

43. Yukinaga, $\mathrm{H}$. et al. Identification of cytochrome P450 3A4 modification site with reactive metabolite using linear ion trap-Fourier transform mass spectrometry. Chem. Res. Toxicol. 20, 1373-1378 (2007).

44. Schenkman, J. B., Sligar, S. G. \& Cinti, D. L. Substrate interaction with cytochrome P-450. Pharmacol. Ther. 12, 43-71 (1981).

45. Locuson, C. W., Hutzler, J. M. \& Tracy, T. S. Visible Spectra of Type II Cytochrome P450Drug Complexes: Evidence that 'Incomplete' Heme Coordination Is Common. Drug Metab. Dispos. 35, 614-622 (2007). 
46. Jett, J. E. et al. Measurement of Electron Transfer through Cytochrome P450 Protein on Nanopillars and the Effect of Bound Substrates. J. Am. Chem. Soc. 135, 3834-3840 (2013).

47. Korzekwa, K. R. et al. Evaluation of Atypical Cytochrome P450 Kinetics with TwoSubstrate Models: Evidence That Multiple Substrates Can Simultaneously Bind to Cytochrome P450 Active Sites†. Biochemistry (Mosc.) 37, 4137-4147 (1998).

48. Hummel, M. A., Gannett, P. M., Aguilar, J. S. \& Tracy, T. S. Effector-Mediated Alteration of Substrate Orientation in Cytochrome P450 2C9†. Biochemistry (Mosc.) 43, 7207-7214 (2004).

49. Roberts, A. G., Campbell, A. P. \& Atkins, W. M. The Thermodynamic Landscape of Testosterone Binding to Cytochrome P450 3A4: Ligand Binding and Spin State Equilibria†. Biochemistry (Mosc.) 44, 1353-1366 (2005).

50. Hutzler, J. M., Kolwankar, D., Hummel, M. A. \& Tracy, T. S. Activation of CYP2C9Mediated Metabolism by a Series of Dapsone Analogs: Kinetics and Structural Requirements. Drug Metab. Dispos. 30, 1194-1200 (2002).

51. Atkins, W. M., Wang, R. W. \& Lu, A. Y. H. Allosteric Behavior in Cytochrome P450Dependent in Vitro Drug-Drug Interactions: A Prospective Based on Conformational Dynamics. Chem. Res. Toxicol. 14, 338-347 (2001).

52. Hill, J. R. in Current Protocols in Pharmacology (John Wiley \& Sons, Inc., 2001). at <http://onlinelibrary.wiley.com/doi/10.1002/0471141755.ph0708s23/abstract>

53. Newton, D. J., Wang, R. W. \& Lu, A. Y. Cytochrome P450 inhibitors. Evaluation of specificities in the in vitrometabolism of therapeutic agents by human liver microsomes. Drug Metab. Dispos. 23, 154-158 (1995).

54. Bourrié, M., Meunier, V., Berger, Y. \& Fabre, G. Cytochrome P450 isoform inhibitors as a tool for the investigation of metabolic reactions catalyzed by human liver microsomes. $J$. Pharmacol. Exp. Ther. 277, 321-332 (1996). 
55. Ahn, T., Guengerich, F. P. \& Yun, C.-H. Membrane Insertion of Cytochrome P450 1A2 Promoted by Anionic Phospholipids. Biochemistry (Mosc.) 37, 12860-12866 (1998).

56. Imaoka, S., Imai, Y., Shimada, T. \& Funae, Y. Role of phospholipids in reconstituted cytochrome P450 3A form and mechanism of their activation of catalytic activity. Biochemistry (Mosc.) 31, 6063-6069 (1992).

57. Causey, K. M., Eyer, C. S. \& Backes, W. L. Dual role of phospholipid in the reconstitution of cytochrome P-450 LM2-dependent activities. Mol. Pharmacol. 38, 134-142 (1990).

58. Brian Houston, J. Utility of in vitro drug metabolism data in predicting in vivo metabolic clearance. Biochem. Pharmacol. 47, 1469-1479 (1994).

59. Cornish-Bowden, A. A simple graphical method for determining the inhibition constants of mixed, uncompetitive and non-competitive inhibitors (Short Communication). Biochem. J. 137, 143-144 (1974).

60. T Kakkar, H. B. Estimation of $\mathrm{Ki}$ in a competitive enzyme-inhibition model: comparisons among three methods of data analysis. Drug Metab. Dispos. Biol. Fate Chem. 27, 756-62 (1999).

61. Geng, W. A Method for Identification of Inhibition Mechanism and Estimation of Ki in in Vitro Enzyme Inhibition Study. Drug Metab. Dispos. 31, 1456-1457 (2003).

62. Dean, W. L. \& Gray, R. D. Relationship between state of aggregation and catalytic activity for cytochrome P-450LM2 and NADPH-cytochrome P-450 reductase. J. Biol. Chem. 257, 14679-14685 (1982).

63. Wagner, S. L., Dean, W. L. \& Gray, R. D. Effect of a zwitterionic detergent on the state of aggregation and catalytic activity of cytochrome P-450LM2 and NADPH-cytochrome P-450 reductase. J. Biol. Chem. 259, 2390-2395 (1984).

64. Hazai, E. \& Kupfer, D. Interactions Between Cyp2c9 and Cyp2c19 in Reconstituted Binary Systems Influence Their Catalytic Activity: Possible Rationale for the Inability of Cyp2c19 
to Catalyze Methoxychlor Demethylation in Human Liver Microsomes. Drug Metab. Dispos. 33, 157-164 (2005).

65. Tan, Y., Patten, C. J., Smith, T. \& Yang, C. S. Competitive interactions between cytochromes P450 2A6 and 2E1 for NADPH-cytochrome P450 oxidoreductase in the microsomal membranes produced by a baculovirus expression system. Arch. Biochem. Biophys. 342, 82-91 (1997).

66. Backes, W. L., Batie, C. J. \& Cawley, G. F. Interactions among P450 Enzymes When Combined in Reconstituted Systems: Formation of a 2B4-1A2 Complex with a High Affinity for NADPH-Cytochrome P450 Reductase†. Biochemistry (Mosc.) 37, 1285212859 (1998).

67. Alston, K., Robinson, R. C., Park, S. S., Gelboin, H. V. \& Friedman, F. K. Interactions among cytochromes P-450 in the endoplasmic reticulum. Detection of chemically crosslinked complexes with monoclonal antibodies. J. Biol. Chem. 266, 735-739 (1991).

68. Kaminsky, L. S. \& Guengerich, F. P. Cytochrome P-450 isozyme/isozyme functional interactions and NADPH-cytochrome $\mathrm{P}-450$ reductase concentrations as factors in microsomal metabolism of warfarin. Eur. J. Biochem. 149, 479-489 (1985).

69. Kanaeva, I. P. et al. Comparative study of monomeric reconstituted and membrane microsomal monooxygenase systems of the rabbit liver. I. Properties of NADPHcytochrome P450 reductase and cytochrome P450 LM2 (2B4) monomers. Arch. Biochem. Biophys. 298, 395-402 (1992).

70. Kanaeva, I. P. et al. Comparative study of monomeric reconstituted and membrane microsomal monooxygenase systems of the rabbit liver. II. Kinetic parameters of reductase and monooxygenase reactions. Arch. Biochem. Biophys. 298, 403-412 (1992).

71. Sevrukova, I. F. et al. Catalytic activity of cytochrome P4501A2 in reconstituted system with Emulgen 913. Arch. Biochem. Biophys. 311, 133-143 (1994). 
72. Myasoedova, K. N., Arutyunyan, A. M. \& Magretova, N. N. Phenomenon of activation of cytochrome P450 by nonionic detergents. Biosci. Rep. 26, 69-78 (2006).

73. Kelley, R. W., Reed, J. R. \& Backes, W. L. Effects of lonic Strength on the Functional Interactions between CYP2B4 and CYP1A2†. Biochemistry (Mosc.) 44, 2632-2641 (2005).

74. Kelley, R. W., Cheng, D. \& Backes, W. L. Heteromeric Complex Formation between CYP2E1 and CYP1A2: Evidence for the involvement of electrostatic interactions. Biochemistry (Mosc.) 45, 15807-15816 (2006).

75. Phillips, A. H. \& Langdon, R. G. Hepatic Triphosphopyridine Nucleotide-Cytochrome c Reductase: Isolation, Characterization, and Kinetic Studies. J. Biol. Chem. 237, 26522660 (1962).

76. Cosme, J. \& Johnson, E. F. Engineering Microsomal Cytochrome P450 $2 \mathrm{C} 5$ to Be a Soluble, Monomeric Enzyme Mutations That Alter Aggregation, Phospholipid Dependence of Catalysis, and Membrane Binding. J. Biol. Chem. 275, 2545-2553 (2000).

77. Hu, G., Johnson, E. F. \& Kemper, B. CYP2C8 Exists as a Dimer in Natural Membranes. Drug Metab. Dispos. 38, 1976-1983 (2010).

78. Subramanian, M., Tam, H., Zheng, H. \& Tracy, T. S. CYP2C9-CYP3A4 Protein-Protein Interactions: Role of the Hydrophobic N Terminus. Drug Metab. Dispos. 38, 1003-1009 (2010).

79. Szczesna-Skorupa, E., Mallah, B. \& Kemper, B. Fluorescence Resonance Energy Transfer Analysis of Cytochromes P450 2C2 and 2E1 Molecular Interactions in Living Cells. J. Biol. Chem. 278, 31269-31276 (2003).

80. Ozalp, C., Szczesna-Skorupa, E. \& Kemper, B. Bimolecular Fluorescence Complementation Analysis of Cytochrome P450 2c2, 2e1, and Nadph-Cytochrome P450 Reductase Molecular Interactions in Living Cells. Drug Metab. Dispos. 33, 1382-1390 (2005). 
81. Reed, J. R. \& Backes, W. L. Formation of P450•P450 Complexes and Their Effect on P450 Function. Pharmacol. Ther. 133, 299-310 (2012).

82. Davis, J. J. et al. Molecular bioelectronics. J. Mater. Chem. 15, 2160-2174 (2005).

83. Finkelstein, J. Metalloproteins. Nature 460, 813-813 (2009).

84. Lu, Y., Yeung, N., Sieracki, N. \& Marshall, N. M. Design of functional metalloproteins. Nature 460, 855-862 (2009).

85. Rasor, J. P. \& Voss, E. Enzyme-catalyzed processes in pharmaceutical industry. Appl. Catal. Gen. 221, 145-158 (2001).

86. van Beilen, J. B., Duetz, W. A., Schmid, A. \& Witholt, B. Practical issues in the application of oxygenases. Trends Biotechnol. 21, 170-177 (2003).

87. Vilker, V. L., Reipa, V., Mayhew, M. \& Holden, M. J. Challenges in capturing oxygenase activity in vitro. J. Am. Oil Chem. Soc. 76, 1283-1289 (1999).

88. Zaks, A. \& Dodds, D. R. Application of biocatalysis and biotransformations to the synthesis of pharmaceuticals. Drug Discov. Today 2, 513-531 (1997).

89. Schulze, B. \& Wubbolts, M. G. Biocatalysis for industrial production of fine chemicals. Curr. Opin. Biotechnol. 10, 609-615 (1999).

90. Tao, J. (Alex), Lin, G.-Q. \& Liese, A. Biocatalysis for the Pharmaceutical Industry: Discovery, Development, and Manufacturing. (John Wiley \& Sons, 2009).

91. Lamb, S. B., Lamb, D. C., Kelly, S. L. \& Stuckey, D. C. Cytochrome P450 immobilisation as a route to bioremediation/biocatalysis. FEBS Lett. 431, 343-346 (1998).

92. Leung, A., Shankar, P. M. \& Mutharasan, R. A review of fiber-optic biosensors. Sens. Actuators B Chem. 125, 688-703 (2007).

93. Wolfbeis, O. S. Fiber-Optic Chemical Sensors and Biosensors. Anal. Chem. 80, 42694283 (2008).

94. Kimmel, D. W., LeBlanc, G., Meschievitz, M. E. \& Cliffel, D. E. Electrochemical Sensors and Biosensors. Anal. Chem. 84, 685-707 (2012). 
95. Kuila, T. et al. Recent advances in graphene-based biosensors. Biosens. Bioelectron. 26, 4637-4648 (2011).

96. Wang, J. Carbon-Nanotube Based Electrochemical Biosensors: A Review. Electroanalysis 17, 7-14 (2005).

97. Carrara, S., Baj-Rossi, C., Boero, C. \& De Micheli, G. Do Carbon Nanotubes contribute to Electrochemical Biosensing? Electrochimica Acta 128, 102-112 (2014).

98. Baj-Rossi, C., De, G. \& Carrar, S. in Biosensors - Emerging Materials and Applications (ed. Serra, P. A.) (InTech, 2011). at <http://www.intechopen.com/books/biosensors-emergingmaterials-and-applications/p450-based-nano-bio-sensors-for-personalized-medicine>

99. Palchetti, I. \& Mascini, M. Electroanalytical biosensors and their potential for food pathogen and toxin detection. Anal. Bioanal. Chem. 391, 455-471 (2008).

100. Ligler, F. S. et al. Array biosensor for detection of toxins. Anal. Bioanal. Chem. 377, 469477 (2003).

101. Carrara, S., Shumyantseva, V. V., Archakov, A. I. \& Samorì, B. Screen-printed electrodes based on carbon nanotubes and cytochrome P450scc for highly sensitive cholesterol biosensors. Biosens. Bioelectron. 24, 148-150 (2008).

102. Baj-Rossi, C., Micheli, G. D. \& Carrara, S. Electrochemical Detection of Anti-Breast-Cancer Agents in Human Serum by Cytochrome P450-Coated Carbon Nanotubes. Sensors 12, 6520-6537 (2012).

103. Yeh, P. \& Kuwana, T. Reversible Electrode Reaction of Cytochrome $<i>c</ l>$. Chem. Lett. 6, 1145-1148 (1977).

104. Eddowes, M. J. \& Hill, H. A. O. Novel method for the investigation of the electrochemistry of metalloproteins: cytochrome c. J. Chem. Soc. Chem. Commun. 771b-772 (1977). doi:10.1039/C3977000771B

105. Scheller, F. et al. Electrochemical investigations on the oxygen activation by cytochrome P-450. Acta Biol. Med. Ger. 38, 503-509 (1979). 
106. Yang, M. et al. Electrocatalytic Drug Metabolism by CYP2C9 Bonded to A Self-Assembled Monolayer-Modified Electrode. Drug Metab. Dispos. 37, 892-899 (2009).

107. Aviram, A. \& Ratner, M. A. Molecular rectifiers. Chem. Phys. Lett. 29, 277-283 (1974).

108. Salemme, F. R. in Bionanotechnology (eds. Renugopalakrishnan, V. \& Lewis, R. V.) 1-8 (Springer Netherlands, 2006). at <http://link.springer.com/chapter/10.1007/978-1-40204375-8_1>

109. Saga, Y., Watanabe, T., Koyama, K. \& Miyasaka, T. Mechanism of Photocurrent Generation from Bacteriorhodopsin on Gold Electrodes. J. Phys. Chem. B 103, 234-238 (1999).

110. Maruccio, G. et al. Towards Protein Field-Effect Transistors: Report and Model of a Prototype. Adv. Mater. 17, 816-822 (2005).

111. Zhang, T. et al. All-optical logic gates using bacteriorhodopsin films. Opt. Eng. 39, 527-534 (2000).

112. Chen, Z. \& Birge, R. R. Protein-based artificial retinas. Trends Biotechnol. 11, 292-300 (1993).

113. Aguilella, V. M. \& Alcaraz, A. Nanobiotechnology: A fluid approach to simple circuits. Nat. Nanotechnol. 4, 403-404 (2009).

114. Ciesielski, P. N. et al. Photosystem I - Based biohybrid photoelectrochemical cells. Bioresour. Technol. 101, 3047-3053 (2010).

115. Ditto, W. L. Chaos in neural systems: from epilepsy to neurocomputing. in Proceedings of the 25th Annual International Conference of the IEEE Engineering in Medicine and Biology Society, 2003 4, 3830-3833 Vol.4 (2003).

116. Ha, S., Wee, Y. \& Kim, J. Nanobiocatalysis for Enzymatic Biofuel Cells. Top. Catal. 55, $1181-1200$ (2012).

117. Reshetilov, A. N. Biosensors and biofuel cells: Research focused on practical application (Review). Appl. Biochem. Microbiol. 51, 264-269 (2015). 
118. Calabrese Barton, S., Gallaway, J. \& Atanassov, P. Enzymatic Biofuel Cells for Implantable and Microscale Devices. Chem. Rev. 104, 4867-4886 (2004).

119. Farahi, R. H., Ferrell, T. L., Guiseppi-Elie, A. \& Hansen, P. Integrated electronics platforms for wireless implantable biosensors. in Life Science Systems and Applications Workshop, 2007. LISA 2007. IEEE/NIH 27-30 (2007). doi:10.1109/LSSA.2007.4400876

120. Guiseppi-Elie, A., Brahim, S., Slaughter, G. \& Ward, K. R. Design of a subcutaneous implantable biochip for monitoring of glucose and lactate. IEEE Sens. J. 5, 345-355 (2005).

121. Berna, A. Z., Anderson, A. R. \& Trowell, S. C. Bio-Benchmarking of Electronic Nose Sensors. PLOS ONE 4, e6406 (2009).

122. Sligar, S. G. \& Salemme, F. R. Protein engineering for molecular electronics. Curr. Biol. 2, 494 (1992).

123. Li, W. et al. Temperature and Force Dependence of Nanoscale Electron Transport via the Cu Protein Azurin. ACS Nano 6, 10816-10824 (2012).

124. Amdursky, N. et al. Electronic Transport via Proteins. Adv. Mater. 26, 7142-7161 (2014).

125. Wierzbinski, E. et al. The Single-Molecule Conductance and Electrochemical ElectronTransfer Rate Are Related by a Power Law. ACS Nano 7, 5391-5401 (2013).

126. Venkatramani, R., Wierzbinski, E., Waldeck, D. H. \& Beratan, D. N. Breaking the simple proportionality between molecular conductances and charge transfer rates. Faraday Discuss. 174, 57-78 (2014).

127. Winkler, J. R., Dunn, A. R., Hess, C. R. \& Gray, H. B. in Bioinorganic Electrochemistry (eds. Hammerich, O. \& Ulstrup, J.) 1-23 (Springer Netherlands, 2008). at <http://link.springer.com/chapter/10.1007/978-1-4020-6500-2_1>

128. Bianco, P. in Encyclopedia of Electrochemistry (Wiley-VCH Verlag GmbH \& Co. KGaA, 2007). at <http://onlinelibrary.wiley.com/doi/10.1002/9783527610426.bard090000/abstract> 
129. Carrara, S., Cavallini, A., Garg, A. \& De Micheli, G. Dynamical spot queries to improve specificity in $\mathrm{P} 450$ s based multi-drugs monitoring. in ICME International Conference on Complex Medical Engineering, 2009. CME 1-6 (2009). doi:10.1109/ICCME.2009.4906648

130. Talbert, J. N. \& Goddard, J. M. Enzymes on material surfaces. Colloids Surf. B Biointerfaces 93, 8-19 (2012).

131. Secundo, F. Conformational changes of enzymes upon immobilisation. Chem. Soc. Rev. 42, 6250-6261 (2013).

132. Malmsten, M. Formation of Adsorbed Protein Layers. J. Colloid Interface Sci. 207, 186199 (1998).

133. Kazlauskaite, J., Westlake, A. C. G., Wong, L.-L. \& Hill, H. A. O. Direct electrochemistry of cytochrome P450cam. Chem. Commun. 2189-2190 (1996). doi:10.1039/CC9960002189

134. Lo, K. K.-W., Wong, L.-L. \& Hill, H. A. O. Surface-modified mutants of cytochrome P450cam: enzymatic properties and electrochemistry. FEBS Lett. 451, 342-346 (1999).

135. Bistolas, N., Wollenberger, U., Jung, C. \& Scheller, F. W. Cytochrome P450 biosensors-a review. Biosens. Bioelectron. 20, 2408-2423 (2005).

136. Lei, C., Wollenberger, U., Jung, C. \& Scheller, F. W. Clay-Bridged Electron Transfer between Cytochrome P450cam and Electrode. Biochem. Biophys. Res. Commun. 268, 740-744 (2000).

137. Niki, K., In:, Bard, A. J., Stratmann, M. \& Wilson, G. Bioelectrochemistry in Encyclopedia of Electrochemistry. 9, (Wiley-VCH, Weinheim, 2002).

138. Sallez, Y., Bianco, P. \& Lojou, E. Electrochemical behavior of c-type cytochromes at claymodified carbon electrodes: a model for the interaction between proteins and soils. $\mathrm{J}$. Electroanal. Chem. 493, 37-49 (2000).

139. Shumyantseva, V. V. et al. Direct Electron Transfer of Cytochrome P450 2B4 at Electrodes Modified with Nonionic Detergent and Colloidal Clay Nanoparticles. Anal. Chem. 76, 60466052 (2004). 
140. Zhang, Z., Nassar, A.-E. F., Lu, Z., Schenkman, J. B. \& Rusling, J. F. Direct electron injection from electrodes to cytochromeP450cam in biomembrane-like films. J. Chem. Soc. Faraday Trans. 93, 1769-1774 (1997).

141. Shukla, A., Gillam, E. M., Mitchell, D. J. \& Bernhardt, P. V. Direct electrochemistry of enzymes from the cytochrome P450 2C family. Electrochem. Commun. 7, 437-442 (2005).

142. Bayburt, T. H. \& Sligar, S. G. Single-molecule height measurements on microsomal cytochrome P450 in nanometer-scale phospholipid bilayer disks. Proc. Natl. Acad. Sci. U. S. A. 99, 6725-6730 (2002).

143. Davydov, D. R., Fernando, H., Baas, B. J., Sligar, S. G. \& Halpert, J. R. Kinetics of dithionite-dependent reduction of cytochrome P450 3A4: heterogeneity of the enzyme caused by its oligomerization. Biochemistry (Mosc.) 44, 13902-13913 (2005).

144. Munge, B., Estavillo, C., Schenkman, J. B. \& Rusling, J. F. Optimization of Electrochemical and Peroxide-Driven Oxidation of Styrene with Ultrathin Polyion Films Containing Cytochrome P450cam and Myoglobin. ChemBioChem 4, 82-89 (2003).

145. Estavillo, C., Lu, Z., Jansson, I., Schenkman, J. B. \& Rusling, J. F. Epoxidation of styrene by human cyt P450 1A2 by thin film electrolysis and peroxide activation compared to solution reactions. Biophys. Chem. 104, 291-296 (2003).

146. Sultana, N., Schenkman, J. B. \& Rusling, J. F. Protein Film Electrochemistry of Microsomes Genetically Enriched in Human Cytochrome P450 Monooxygenases. J. Am. Chem. Soc. 127, 13460-13461 (2005).

147. Krishnan, S., Wasalathanthri, D., Zhao, L., Schenkman, J. B. \& Rusling, J. F. Efficient Bioelectronic Actuation of the Natural Catalytic Pathway of Human Metabolic Cytochrome P450s. J. Am. Chem. Soc. 133, 1459-1465 (2011).

148. Shumyantseva, V. V. et al. Electrochemical Reduction of Flavocytochromes $2 \mathrm{~B} 4$ and $1 \mathrm{~A} 2$ and Their Catalytic Activity. Arch. Biochem. Biophys. 377, 43-48 (2000). 
149. Panicco, P., Dodhia, V. R., Fantuzzi, A. \& Gilardi, G. Enzyme-Based Amperometric Platform to Determine the Polymorphic Response in Drug Metabolism by Cytochromes P450. Anal. Chem. 83, 2179-2186 (2011).

150. Udit, A. K. \& Gray, H. B. Electrochemistry of heme-thiolate proteins. Biochem. Biophys. Res. Commun. 338, 470-476 (2005).

151. González-Granados, Z. et al. Formation of Mixed Monolayers from 11Mercaptoundecanoic Acid and Octanethiol on Au(111) Single Crystal Electrode under Electrochemical Control. J. Phys. Chem. C 117, 24307-24316 (2013).

152. Yan, C., Zharnikov, M., Gölzhäuser, A. \& Grunze, M. Preparation and Characterization of Self-Assembled Monolayers on Indium Tin Oxide. Langmuir 16, 6208-6215 (2000).

153. Love, J. C., Estroff, L. A., Kriebel, J. K., Nuzzo, R. G. \& Whitesides, G. M. Self-Assembled Monolayers of Thiolates on Metals as a Form of Nanotechnology. Chem. Rev. 105, 11031170 (2005).

154. Arya, S. K., Solanki, P. R., Datta, M. \& Malhotra, B. D. Recent advances in self-assembled monolayers based biomolecular electronic devices. Biosens. Bioelectron. 24, 2810-2817 (2009).

155. Mak, L. H., Sadeghi, S. J., Fantuzzi, A. \& Gilardi, G. Control of Human Cytochrome P450 2E1 Electrocatalytic Response as a Result of Unique Orientation on Gold Electrodes. Anal. Chem. 82, 5357-5362 (2010).

156. Cui, X. D. et al. Making electrical contacts to molecular monolayers. Nanotechnology 13, 5 (2002).

157. Wong, L. S., Khan, F. \& Micklefield, J. Selective Covalent Protein Immobilization: Strategies and Applications. Chem. Rev. 109, 4025-4053 (2009).

158. Gannett, P. M. et al. Preparation, Characterization, and Substrate Metabolism of GoldImmobilized Cytochrome P450 2C9. J Am Chem Soc 128, 8374-8375 (2006). 
159. Zheng, Z. \& Gunner, M. R. Analysis of the electrochemistry of hemes with Ems spanning $800 \mathrm{mV}$. Proteins 75, 719-734 (2009).

160. Lang, D. \& Böcker, R. Highly sensitive and specific high-performance liquid chromatographic analysis of 7-hydroxywarfarin, a marker for human cytochrome P-4502C9 activity. J. Chromatogr. B Biomed. Appl. 672, 305-309 (1995).

161. Fantuzzi, A. et al. A New Standardized Electrochemical Array for Drug Metabolic Profiling with Human Cytochromes P450. Anal. Chem. 83, 3831-3839 (2011).

162. Alessandrini, A., Corni, S. \& Facci, P. Unravelling single metalloprotein electron transfer by scanning probe techniques. Phys. Chem. Chem. Phys. PCCP 8, 4383-4397 (2006).

163. Artés, J. M., Díez-Pérez, I., Sanz, F. \& Gorostiza, P. Direct Measurement of Electron Transfer Distance Decay Constants of Single Redox Proteins by Electrochemical Tunneling Spectroscopy. ACS Nano (2011). doi:10.1021/nn103236e

164. Chi, Q., Farver, O. \& Ulstrup, J. Long-range protein electron transfer observed at the single-molecule level: In situ mapping of redox-gated tunneling resonance. Proc. Natl. Acad. Sci. U. S. A. 102, 16203-16208 (2005).

165. Reed, M. A., Zhou, C., Muller, C. J., Burgin, T. P. \& Tour, J. M. Conductance of a Molecular Junction. Science 278, 252-254 (1997).

166. Kergueris, C. et al. Electron transport through a metal-molecule-metal junction. Phys. Rev. B 59, 12505-12513 (1999).

167. Park, J. et al. Coulomb blockade and the Kondo effect in single-atom transistors. Nature 417, 722-725 (2002).

168. Li, D., Gannet, P. M. \& Lederman, D. An investigation into the feasibility of myoglobinbased single-electron transistors. Nanotechnology 23, 395705 (2012).

169. van der Zant, H. S. J. et al. Molecular three-terminal devices: fabrication and measurements. Faraday Discuss. 131, 347-356 (2006). 
170. Smaali, K., Clément, N., Patriarche, G. \& Vuillaume, D. Conductance Statistics from a Large Array of Sub-10 nm Molecular Junctions. ACS Nano 6, 4639-4647 (2012).

171. Rabe, M., Verdes, D. \& Seeger, S. Understanding protein adsorption phenomena at solid surfaces. Adv. Colloid Interface Sci. 162, 87-106 (2011).

172. Fleming, B. D., Johnson, D. L., Bond, A. M. \& Martin, L. L. Recent progress in cytochrome P450 enzyme electrochemistry. Expert Opin. Drug Metab. Toxicol. 2, 581-589 (2006).

173. Billsten, P., Wahlgren, M., Arnebrant, T., McGuire, J. \& Elwing, H. Structural Changes of T4 Lysozyme upon Adsorption to Silica Nanoparticles Measured by Circular Dichroism. J. Colloid Interface Sci. 175, 77-82 (1995).

174. Karlsson, M. \& Carlsson, U. Protein adsorption orientation in the light of fluorescent probes: mapping of the interaction between site-directly labeled human carbonic anhydrase II and silica nanoparticles. Biophys. J. 88, 3536-3544 (2005).

175. Hutzler, J. M., Wienkers, L. C., Wahlstrom, J. L., Carlson, T. J. \& Tracy, T. S. Activation of cytochrome P450 2C9-mediated metabolism: mechanistic evidence in support of kinetic observations. Arch. Biochem. Biophys. 410, 16-24 (2003).

176. Nicolini, C. et al. P450scc Engineering and Nanostructuring for Cholesterol Sensing. Langmuir 17, 3719-3726 (2001).

177. Homola, J., Yee, S. S. \& Gauglitz, G. Surface plasmon resonance sensors: review. Sens. Actuators B Chem. 54, 3-15 (1999).

178. Sexton, B. A., Feltis, B. N. \& Davis, T. J. Characterisation of gold surface plasmon resonance sensor substrates. Sens. Actuators Phys. 141, 471-475 (2008).

179. Ivanov, A. S., Gnedenko, O. V., Molnar, A. A., Archakov, A. I. \& Podust, L. M. FMN Binding Site of Yeast NADPH-Cytochrome P450 Reductase Exposed at the Surface Is Highly Specific. ACS Chem. Biol. 5, 767-776 (2010). 
180. Ivanov, Y. D. et al. Molecular Recognition in the P450cam Monooxygenase System: Direct Monitoring of Protein-Protein Interactions by Using Optical Biosensor. Arch. Biochem. Biophys. 391, 255-264 (2001).

181. McNay, G., Eustace, D., Smith, W. E., Faulds, K. \& Graham, D. Surface-enhanced Raman scattering (SERS) and surface-enhanced resonance Raman scattering (SERRS): a review of applications. Appl. Spectrosc. 65, 825-837 (2011).

182. Todorovic, S., Jung, C., Hildebrandt, P. \& Murgida, D. H. Conformational transitions and redox potential shifts of cytochrome P450 induced by immobilization. J. Biol. Inorg. Chem. JBIC Publ. Soc. Biol. Inorg. Chem. 11, 119-127 (2006).

183. Bonifacio, A. et al. Active-site structure, binding and redox activity of the heme-thiolate enzyme CYP2D6 immobilized on coated Ag electrodes: a surface-enhanced resonance Raman scattering study. J. Biol. Inorg. Chem. 13, 85-96 (2008).

184. Alessandrini, A., Salerno, M., Frabboni, S. \& Facci, P. Single-metalloprotein wet biotransistor. Appl. Phys. Lett. 86, 133902-133902-3 (2005).

185. Davis, J. J. et al. General Discussion. Faraday Discuss. 116, 67-75 (2000).

186. Davis, J. J. et al. A scanning tunnelling study of immobilised cytochrome P450cam. Faraday Discuss. 116, 15-22 (2000).

187. Djuricic, D., Hill, H. A. O., Lo, K. K.-W. \& Wong, L.-L. A scanning tunneling microscopy (STM) investigation of complex formation between cytochrome P450cam and putidaredoxin. J. Inorg. Biochem. 88, 362-367 (2002).

188. Möller, C., Allen, M., Elings, V., Engel, A. \& Müller, D. J. Tapping-Mode Atomic Force Microscopy Produces Faithful High-Resolution Images of Protein Surfaces. Biophys. J. 77, 1150-1158 (1999).

189. Nussio, M. R. et al. AFM study of the interaction of cytochrome P450 2C9 with phospholipid bilayers. Chem. Phys. Lipids 163, 182-189 (2010). 
190. Archakov, A. I. \& Ivanov, Y. D. Application of AFM and optical biosensor for investigation of complexes formed in P450-containing monooxygenase systems. Biochim. Biophys. Acta BBA - Proteins Proteomics 1814, 102-110 (2011).

191. Matsuura, S., Fujii-Kuriyama, Y. \& Tashiro, Y. Immunoelectron microscope localization of cytochrome P-450 on microsomes and other membrane structures of rat hepatocytes. J. Cell Biol. 78, 503-519 (1978).

192. Jungermann, K. Zonation of metabolism and gene expression in liver. Histochem. Cell Biol. 103, 81-91 (1995).

193. Backes, W. L. \& Kelley, R. W. Organization of multiple cytochrome P450s with NADPHcytochrome P450 reductase in membranes. Pharmacol. Ther. 98, 221-233 (2003).

194. Subramanian, M., Low, M., Locuson, C. W. \& Tracy, T. S. CYP2D6-CYP2C9 ProteinProtein Interactions and Isoform-Selective Effects on Substrate Binding and Catalysis. Drug Metab. Dispos. 37, 1682-1689 (2009).

195. Davydov, D. R., Petushkova, N. A., Bobrovnikova, E. V., Knyushko, T. V. \& Dansette, P. Association of cytochromes P450 1A2 and 2B4: are the interactions between different P450 species involved in the control of the monooxygenase activity and coupling? Adv. Exp. Med. Biol. 500, 335-338 (2001).

196. Cawley, G. F., Zhang, S., Kelley, R. W. \& Backes, W. L. Evidence Supporting the Interaction of CYP2B4 and CYP1A2 in Microsomal Preparations. Drug Metab. Dispos. 29, 1529-1534 (2001).

197. Cawley, G. F., Batie, C. J. \& Backes, W. L. Substrate-dependent competition of different P450 isozymes for limiting NADPH-cytochrome P450 reductase. Biochemistry (Mosc.) 34, 1244-1247 (1995).

198. Li, D. N. et al. Competition Between Cytochrome P-450 Isozymes for NADPH-Cytochrome P-450 Oxidoreductase Affects Drug Metabolism. J. Pharmacol. Exp. Ther. 289, 661-667 (1999). 
199. Jamakhandi, A. P., Kuzmic, P., Sanders, D. E. \& Miller, G. P. Global Analysis of ProteinProtein Interactions Reveals Multiple Cytochrome P450 2E1-Reductase Complexes. Biochemistry (Mosc.) 46, 10192-10201 (2007).

200. Davydov, D. R., Knyushko, T. V. \& Hoa, G. H. High pressure induced inactivation of ferrous cytochrome P-450 LM2 (IIB4) CO complex: evidence for the presence of two conformers in the oligomer. Biochem. Biophys. Res. Commun. 188, 216-221 (1992).

201. Davydov, D. R. et al. Electron transfer in the complex of membrane-bound human cytochrome P450 3A4 with the flavin domain of P450BM-3: the effect of oligomerization of the heme protein and intermittent modulation of the spin equilibrium. Biochim. Biophys. Acta 1797, 378 (2010).

202. Dutton, D. R., McMillen, S. K., Sonderfan, A. J., Thomas, P. E. \& Parkinson, A. Studies on the rate-determining factor in testosterone hydroxylation by rat liver microsomal cytochrome P-450: evidence against cytochrome P-450 isozyme:isozyme interactions. Arch. Biochem. Biophys. 255, 316-328 (1987).

203. Wollenberg, L. A. et al. Selective filling of nanowells in nanowell arrays fabricated using polystyrene nanosphere lithography with cytochrome P450 enzymes. Nanotechnology 23, 385101 (2012).

204. Bostick, C. D., Flora, D. R., Gannett, P. M., Tracy, T. S. \& Lederman, D. Nanoscale electron transport measurements of immobilized cytochrome P450 proteins. Nanotechnology 26, 155102 (2015).

205. Cheesman, M. J., Baer, B. R., Zheng, Y.-M., Gillam, E. M. J. \& Rettie, A. E. Rabbit CYP4B1 engineered for high-level expression in Escherichia coli: ligand stabilization and processing of the $\mathrm{N}$-terminus and heme prosthetic group. Arch. Biochem. Biophys. 416, 17-24 (2003).

206. Williams, P. A. et al. Crystal structure of human cytochrome P450 2C9 with bound warfarin. Nature 424, 464-468 (2003). 
207. Wester, M. R. et al. The Structure of Human Cytochrome P450 2C9 Complexed with Flurbiprofen at 2.0-Å Resolution. J. Biol. Chem. 279, 35630-35637 (2004).

208. Eargle, J., Wright, D. \& Luthey-Schulten, Z. Multiple Alignment of protein structures and sequences for VMD. Bioinformatics 22, 504-506 (2006).

209. Rowland, P. et al. Crystal Structure of Human Cytochrome P450 2D6. J. Biol. Chem. 281, 7614-7622 (2006).

210. Yano, J. K. et al. The Structure of Human Microsomal Cytochrome P450 3A4 Determined by X-ray Crystallography to 2.05-Å Resolution. J. Biol. Chem. 279, 38091-38094 (2004).

211. Hamdane, D. et al. Structure and Function of an NADPH-Cytochrome P450 Oxidoreductase in an Open Conformation Capable of Reducing Cytochrome P450. J. Biol. Chem. 284, 11374-11384 (2009).

212. Tovchigrechko, A. \& Vakser, I. A. GRAMM-X public web server for protein-protein docking. Nucleic Acids Res. 34, W310-W314 (2006).

213. Humphrey, W., Dalke, A. \& Schulten, K. VMD - Visual Molecular Dynamics. J Molec Graph. 14, 33-38 (1996).

214. Cojocaru, V., Balali-Mood, K., Sansom, M. S. P. \& Wade, R. C. Structure and Dynamics of the Membrane-Bound Cytochrome P450 2C9. PLoS Comput. Biol. 7, 1-14 (2011).

215. Shen, A. L. \& Kasper, C. B. Role of Acidic Residues in the Interaction of NADPHCytochrome P450 Oxidoreductase with Cytochrome P450 and Cytochrome c. J. Biol. Chem. 270, 27475-27480 (1995).

216. Hazai, E., Bikádi, Z., Simonyi, M. \& Kupfer, D. Association of Cytochrome P450 Enzymes is a Determining Factor in their Catalytic Activity. J. Comput. Aided Mol. Des. 19, 271-285 (2005).

217. Miller, B. et al. MMPBSA.py: An Efficient Program for End-State Free Energy Calculations. J. Chem. Theory Comput. 8, 3314-3321 (2012).

218. Zhang, W., Hou, T., Schafmeister, C., Ross, W. S. \& Case, D. A. LeAP. 
219. Case, D. A. et al. AMBER 12. (2012).

220. Roe, D. R. \& Cheatham, T. E. PTRAJ and CPPTRAJ: Software for Processing and Analysis of Molecular Dynamics Trajectory Data. J. Chem. Theory Comput. 9, 3084-3095 (2013).

221. Meijerman, I., Sanderson, L. M., Smits, P. H. M., Beijnen, J. H. \& Schellens, J. H. M. Pharmacogenetic screening of the gene deletion and duplications of CYP2D6. Drug Metab. Rev. 39, 45-60 (2007).

222. Cojocaru, V., Winn, P. J. \& Wade, R. C. The ins and outs of cytochrome P450s. Biochim. Biophys. Acta BBA - Gen. Subj. 1770, 390-401 (2007).

223. Cojocaru, V., Balali-Mood, K., Sansom, M. S. P. \& Wade, R. C. Structure and Dynamics of the Membrane-Bound Cytochrome P450 2C9. PLoS Comput Biol 7, e1002152 (2011).

224. Scott, E. E. et al. Structure of Mammalian Cytochrome P450 2B4 Complexed with 4-(4Chlorophenyl)imidazole at 1.9- $\AA$ Resolution INSIGHT INTO THE RANGE OF P450 CONFORMATIONS AND THE COORDINATION OF REDOX PARTNER BINDING. J. Biol. Chem. 279, 27294-27301 (2004).

225. Williams, P. A., Cosme, J., Sridhar, V., Johnson, E. F. \& McRee, D. E. Mammalian Microsomal Cytochrome P450 Monooxygenase: Structural Adaptations for Membrane Binding and Functional Diversity. Mol. Cell 5, 121-131 (2000).

226. Roberts, A. G. et al. Intramolecular Heme Ligation of the Cytochrome P450 2C9 R108H Mutant Demonstrates Pronounced Conformational Flexibility of the B-C Loop Region: Implications for Substrate Binding. Biochemistry (Mosc.) 49, 8700-8708 (2010).

227. Hou, T., Wang, J., Li, Y. \& Wang, W. Assessing the Performance of the MM/PBSA and MM/GBSA Methods. 1. The Accuracy of Binding Free Energy Calculations Based on Molecular Dynamics Simulations. J. Chem. Inf. Model. 51, 69-82 (2011). 
228. Karlsson, R., Michaelsson, A. \& Mattsson, L. Kinetic-Analysis of Monoclonal AntibodyAntigen Interactions with a New Biosensor Based Analytical System. J. Immunol. Methods 145, 229-240 (1991).

229. Myszka, D. G. Survey of the 1998 optical biosensor literature. J. Mol. Recognit. 12, 390408 (1999).

230. Wei, L., Locuson, C. W. \& Tracy, T. S. Polymorphic Variants of CYP2C9: Mechanisms Involved in Reduced Catalytic Activity. Mol. Pharmacol. 72, 1280-1288 (2007).

231. Locuson, C. W., Wienkers, L. C., Jones, J. P. \& Tracy, T. S. CYP2C9 Protein Interactions with Cytochrome b5: Effects on the Coupling of Catalysis. Drug Metab. Dispos. 35, 11741181 (2007).

232. Stepankova, V. et al. Expansion of access tunnels and active-site cavities influence activity of haloalkane dehalogenases in organic cosolvents. Chembiochem Eur. J. Chem. Biol. 14, 890-897 (2013).

233. Gorsky, L. D. \& Coon, M. J. Effects of conditions for reconstitution with cytochrome b5 on the formation of products in cytochrome P-450-catalyzed reactions. Drug Metab. Dispos. 14, 89-96 (1986).

234. Shimada, T., Mernaugh, R. L. \& Guengerich, F. P. Interactions of mammalian cytochrome P450, NADPH-cytochrome P450 reductase, and cytochrome b5 enzymes. Arch. Biochem. Biophys. 435, 207-216 (2005).

235. Ingelman-Sundberg, M. Phospholipids and detergents as effectors in the liver microsomal hydroxylase system. Biochim. Biophys. Acta 488, 225-234 (1977).

236. Hamdane, D. et al. Structure and Function of an NADPH-Cytochrome P450 Oxidoreductase in an Open Conformation Capable of Reducing Cytochrome P450. J. Biol. Chem. 284, 11374-11384 (2009).

237. Davydov, D. R., Kariakin, A. A., Petushkova, N. A. \& Peterson, J. A. Association of Cytochromes P450 with Their Reductases: Opposite Sign of the Electrostatic Interactions 
in P450BM-3 As Compared with the Microsomal 2B4 System. Biochemistry (Mosc.) 39, 6489-6497 (2000).

238. Sevrioukova, I. F., Li, H., Zhang, H., Peterson, J. A. \& Poulos, T. L. Structure of a cytochrome P450-redox partner electron-transfer complex. Proc. Natl. Acad. Sci. 96, 1863-1868 (1999).

239. Tezcan, F. A., Winkler, J. R. \& Gray, H. B. Effects of Ligation and Folding on Reduction Potentials of Heme Proteins. J. Am. Chem. Soc. 120, 13383-13388 (1998).

240. Hlavica, P. Control by substrate of the cytochrome p450-dependent redox machinery: mechanistic insights. Curr. Drug Metab. 8, 594-611 (2007).

241. Denisov, I. G., Grinkova, Y. V., McLean, M. A. \& Sligar, S. G. The One-electron Autoxidation of Human Cytochrome P450 3A4. J. Biol. Chem. 282, 26865-26873 (2007).

242. Johnson, D. L., Lewis, B. C., Elliot, D. J., Miners, J. O. \& Martin, L. L. Electrochemical characterisation of the human cytochrome P450 CYP2C9. Biochem. Pharmacol. 69, 15331541 (2005).

243. Das, A., Grinkova, Y. V. \& Sligar, S. G. Redox Potential Control by Drug Binding to Cytochrome P450 3A4. J. Am. Chem. Soc. 129, 13778-13779 (2007).

244. Sadeghi, S. J. \& Gilardi, G. Chimeric P450 enzymes: activity of artificial redox fusions driven by different reductases for biotechnological applications. Biotechnol. Appl. Biochem. 60, 102-110 (2013).

245. Wei, J. et al. Direct Wiring of Cytochrome c's Heme Unit to an Electrode: Electrochemical Studies. J. Am. Chem. Soc. 124, 9591-9599 (2002).

246. Zhao, J. \& Davis, J. J. Molecular electron transfer of protein junctions characterised by conducting atomic force microscopy. Colloids Surf. B Biointerfaces 40, 189-194 (2005).

247. Hummel, M. A. et al. CYP2C9 genotype-dependent effects on in vitro drug-drug interactions: switching of benzbromarone effect from inhibition to activation in the CYP2C9.3 variant. Mol. Pharmacol. 68, 644-651 (2005). 
248. Nečas, D. \& Klapetek, P. Gwyddion: an open-source software for SPM data analysis. Cent. Eur. J. Phys. 10, 181-188 (2012).

249. Chen, X., Ferrigno, R., Yang, J. \& Whitesides, G. M. Redox Properties of Cytochrome c Adsorbed on Self-Assembled Monolayers: A Probe for Protein Conformation and Orientation. Langmuir 18, 7009-7015 (2002).

250. Karrasch, S., Dolder, M., Schabert, F., Ramsden, J. \& Engel, A. Covalent binding of biological samples to solid supports for scanning probe microscopy in buffer solution. Biophys. J. 65, 2437-2446 (1993).

251. Li, W. et al. Temperature and Force Dependence of Nanoscale Electron Transport via the Cu Protein Azurin. ACS Nano 6, 10816-10824 (2012).

252. Ron, I. et al. Proteins as Electronic Materials: Electron Transport through Solid-State Protein Monolayer Junctions. J. Am. Chem. Soc. 132, 4131-4140 (2010).

253. Zhao, J., Davis, J. J., Sansom, M. S. P. \& Hung, A. Exploring the Electronic and Mechanical Properties of Protein Using Conducting Atomic Force Microscopy. J. Am. Chem. Soc. 126, 5601-5609 (2004).

254. Davis, J. J., Wang, N., Morgan, A., Zhang, T. \& Zhao, J. Metalloprotein tunnel junctions: compressional modulation of barrier height and transport mechanism. Faraday Discuss. 131, 167-179; discussion 205-220 (2006).

255. Frenkel, J. On Pre-Breakdown Phenomena in Insulators and Electronic Semi-Conductors. Phys. Rev. 54, 647-648 (1938).

256. Sandhu, P. et al. Expression of modified human cytochrome P450 1A2 in Escherichia coli: stabilization, purification, spectral characterization, and catalytic activities of the enzyme. Arch. Biochem. Biophys. 309, 168-177 (1994).

257. Davis, J. J., Peters, B. \& Xi, W. Force modulation and electrochemical gating of conductance in a cytochrome. J. Phys. Condens. Matter 20, 374123 (2008). 
258. Si, D. et al. Mechanism of CYP2C9 Inhibition by Flavones and Flavonols. Drug Metab. Dispos. 37, 629-634 (2009).

259. Shet, M. S., Fisher, C. W., Holmans, P. L. \& Estabrook, R. W. Human cytochrome P450 3A4: enzymatic properties of a purified recombinant fusion protein containing NADPHP450 reductase. Proc. Natl. Acad. Sci. U. S. A. 90, 11748-11752 (1993).

260. Kumar, V., Locuson, C. W., Sham, Y. Y. \& Tracy, T. S. Amiodarone Analog-Dependent Effects on CYP2C9-Mediated Metabolism and Kinetic Profiles. Drug Metab. Dispos. 34, 1688-1696 (2006). 


\section{Chapter 6 Back Matter}




\title{
Christopher D. Bostick
}

\author{
cbostick@mix.wvu.edu
}

609-540-3316 (cell)

OBJECTIVE

PhD with biochemical and biophysical skillset seeking a postdoctoral fellowship.

\section{EDUCATION}

\begin{tabular}{|c|c|}
\hline $\begin{array}{l}\text { West Virginia University (PhD) - Morgantown, WV } \\
\text { - Doctor of Philosophy in Pharmaceutical and Pharmacological Sciences } \\
\text { - Field: Pharmaceutical Sciences } \\
\text { - Advisor Dr. Grazyna Szklarz }\end{array}$ & August 2010 - November 2015 \\
\hline $\begin{aligned} \text { Cornell University (BS) - Ithaca, NY } \\
\text { - } \quad \text { Bachelor of Science in Biology } \\
\text { - } \quad \text { Minor in Business }\end{aligned}$ & August 2005 - May 2009 \\
\hline
\end{tabular}

\section{RESEARCH EXPERIENCE}

West Virginia University, Morgantown, WV - Graduate Research Fellow/Assistant

Dissertation: Use of Protein Immobilization to Measure Cytochrome P450 Conduction and Metabolism Kinetics

December 2015

- Studied immobilized Cytochrome P450 metabolism kinetics in the presence of various effectors.

- Created novel nanopillar platforms using Electron Beam Lithography for use in the controlled immobilization and isolation of Cytochrome P450s.

- Interrogated Cytochrome P450 metabolism kinetics through the measurement of electron transfer using Conductive Probe Atomic Force Microscopy.

- Analyzed Cytochrome P450 through top-down and bottom-up mass spectrometry using Thermo QExactive and nanoLC, Xcalibur, and Proteome Discoverer.

- Studied fullerene nanoparticles effect on Cytochrome P450 mediated metabolism.

- Characterized nanoparticles using Dynamic Light Scattering, Atomic Force Microscopy and UV-Vis Spectroscopy.

- Determined binding of fullerene nanoparticles to Cytochrome P450 in silico utilizing molecular modeling (Autodock tools, Amber, Insight, and Gramm)

- Developed assay using Surface Plasmon Resonance (Biacore X100) to obtain binding kinetics of fullerene to Cytochrome P450.

- Designed studies to measure nanoparticle effect on Cytochrome P450 metabolism utilizing High Performance Liquid Chromatography and Empower software.

- Implemented pharmacokinetic fitting using Origin Pro to determine nanoparticle binding constants and mechanism of effect.

- Studied P450 Protein Interactions and metabolism effect through binding analysis, molecular modeling, and metabolism studies.

- Developed assay to measure Cytochrome P450 protein-protein interaction kinetics utilizing Surface Plasmon Resonance (Biacore X100).

- Also utilize various other techniques including SDS Page Gel, Scanning Electron Microscopy, Nuclear Magnetic Resonance and Clean room techniques: Sputtering, Electron beam Evaporation, Goniometer, and Xray Photoelectron Spectroscopy.

- Supervised three undergraduate research assistants.

Weizmann Institute of Science, Rehovot, Israel - Visiting Student

Research in the lab of Dr. David Cahen

December 2012, October 2015

- Performed Atomic Force Microscopy to study Electron Transfer on Cytochrome P450 films.

- Received training on proper Conductive Probe Atomic Force Microscopy techniques and data analysis.

- Employed nano-fabrication techniques to generate and study platforms for single molecule studies of Myoglobin.

West Virginia University, Morgantown, WV - Doctoral Research Rotations Research rotations in the laboratories of Dr. Jason Huber and Dr. Jamaal Mustafa

January 2011 - March 2011 
- Assisted on a project looking for genomic biomarkers that could be used for early stroke diagnosis by studying isolated rat RNA and Lymphocytes.

- Assisted on a project characterizing the role of adenosine regulation of vascular tone in hypertensive mice using Lagendorff heart perfusion.

- Performed dissections to remove and clean mouse hearts.

- Genotyped adenosine receptor knockout mice by real time PCR.

\section{AWARDS \& GRANTS}

- IGERT Fellow August 2013 - Present

- WV NANOSafe Graduate Fellow August 2011- August 2013

- $\quad 2^{\text {nd }}$ Place Poster Presentation WVU School of Pharmacy AAPS Regional Meeting \& Research Forum June 2015

- West Virginia University School of Pharmacy Graduate Student Outstanding Achievement Award April 2015

- 1 st Place Podia Presentation Van Liere Research Day WVU February 2014

- 3rd place finisher in Science Idol at NSF EPSCoR National Conference November 2013

- 3rd Place Poster Presentation WVU School of Pharmacy AAPS Regional Meeting \& Research Forum May 2013

- West Virginia University School of Pharmacy Dean's Travel Award April 2013

- 1st Place Poster Presentation WVU ACS Chemical Symposium March 2013

- 2nd Place Poster Presentation Van Liere Research Day WVU March 2012

\section{PUBLICATIONS}

Jett, J.E., Lederman, D., Wollenberg, L.A., Li, D., Flora, D.R., Bostick, C.D., Tracy, T.S., and Gannett, P.M.

Measurement of Electron Transfer through Cytochrome P450 Protein on Nanopillars and the Effect of Bound

Substrates. J. Am. Chem. Soc. 2013, 135(10), 3834-3840

Bostick, C.D., Gannett, P.M., Flora, D.R., Tracy, T.S., and Lederman, David. Nanoscale Electron Transfer Measurements of Immobilized Cytochrome P450 Proteins. Nanotechnology 2015, 26(15), 155102

Hong, K. L., Imlay, K., Battistella, L., Williams, R.M., Hickey, K.M., Bostick, C.D., Gannett, P.M., and Sooter, L.J. Selection of Single-Stranded DNA Molecular Recognition Elements Against Exotoxin A using A Novel Decoy-SELEX Method and Sensitive Detection of Exotoxin A in Human Serum. Biomed Res Int. In press.

Bostick C.D*., Hickey, K.M*., Wollenberg L, Flora, D.R., Tracy T.S., \& Gannett PM. Immobilized Cytochrome P450 for monitoring of P450-P450 Interactions and Metabolism. Drug Metabolism and Disposition Pending Revision

Bostick C.D., Ayscue, R, Wang, L., Tracy T.S., \& Gannett PM. Fullerene Nanoparticles effect on Cytochrome P450 Mediated Metabolism. In preparation.

Bostick C.D., Mukhopadhyay, S., Cahen, D., Lederman, D. Towards Bioelectronics with Immobilized Proteins: a review of what we (do not) know. Invited Manuscript for Reports of Progress in Physics. In preparation.

Co first authorship denoted by *

\section{CONFERENCES \& PRESENTATIONS}

Poster Presentation: Bostick C.D., Hickey K.M., Wollenberg L.L., Flora, D.R., Tracy, T.S., \&Gannett PM. "Controlled Immobilization of P450s to Study Protein-Protein Interactions", American Association of Pharmaceutical Scientist Regional Meeting, June 2015 at WVU.

Poster Presentation: Bostick, C.D., Gannett, P.M., Flora, D.R., Tracy, T.S., and Lederman, David. "Nanoscale Electron Transfer Measurements of Immobilized Cytochrome P450 Proteins”, International Symposium on Organic Electronics and Bioelectronics, May 2015 at South Carolina.

Poster Presentation: Bostick C.D., Wang L., Tish WD, \&Gannett PM. Buckminster Fullerene Inhibition of Cytochrome P450 Mediated Metabolism”, American Chemical Society National Meeting, March 2015 at Colorado. 
Poster Presentation: Bostick C.D., Jett J, Lederman D, Tracy TS, \&Gannett PM. "Cytochrome P450 Electron Transfer Measured through Gold Nanopillar Array”, International Biosensing and Bioelectronic Symposium, April 2014 at West Virginia.

Selected Podia Presentation: "Studying Cytochrome P450 Metabolism Kinetics through Measurement of Electron Transfer," Van Liere Memorial Convocation \& HSC Research Day, February 2014 at West Virginia.

Poster Presentation: Bostick C.D., Jett J, Lederman D, Tracy TS, \&Gannett PM. "Probing Electron Transfer in Isolated Cytochrome P450s", NSF EPSCoR National Conference, November 2013 at Tennessee.

Selected Podia Presentation: "'Single Molecule Direct Measurement of Electron Transfer in Cytochrome P450 and the Effect of Bound Substrates", Materials Research Society Spring Conference, April 2013 at California.

Poster Presentation: Bostick C.D., Tish WD, \&Gannett PM. Buckminster Fullerene Inhibition of Cytochrome P450 CYP2C9 Mediated Metabolism”, ACS Chemical Symposium, March 2013 at West Virginia University.

Poster Presentation: Bostick C.D., Jett J, Lederman D, Tracy TS, \&Gannett PM. "Single Molecule Direct Measurement of Electron Transfer in Cytochrome P450s and the Effect of Bound Substrate”, Star Symposium, April 2012 at West Virginia State University.

Poster Presentation: Bostick C.D., Jett J, Lederman D, Tracy TS, \&Gannett PM. "Electron Transfer in Cytochrome P450s in Relation to Various Substrates", Van Liere Memorial Convocation \& HSC Research Day, March 2012 at West Virginia University.

Poster Presentation: Bostick C.D., Wollenberg L, Hickey K, Kabulski J, Wu N, Tracy TS, \& Gannett PM. "Monitoring and Control of P450 Protein-Protein Interactions", Mid-Atlantic Graduate Student Symposium, June 2010 at Duquesne University.

\section{PROFESIONAL AFFILIATIONS}

- Member of The American Society for Pharmacology and Experimental Therapeutics

- Member of American Association of Pharmaceutical Scientist

- Member of Rho Chi Academic Honor Society in Pharmacy

- Member of American Chemical Society

- Member of Materials Research Society

- Member of Alpha Chi Sigma Professional Chemistry Fraternity

\section{LEADERSHIP \& SERVICE}

- School of Pharmacy Graduate Research Advisory Committee - Student Representative August 2014-2015

- WVU Van Liere Conference 2015 Podia Selection Committee and Judge

- West Virginia University AAPS \& ISPOR School of Pharmacy Regional Research Forum 2014

- Executive Planning Committee Lead

- WVU American Association of Pharmaceutical Scientists Chapter Vice President September 2013-2014

- West Virginia Science Bowl 2014, 2015 Volunteer

- Morgantown Boys and Girls Club 2013-2014 Volunteer

- Morgantown Children's Museum 2011-Present Science Days Volunteer

- Chestnut Ridge Church group leader 2013-present

\section{ADDITIONAL EXPERIENCE}

- Certified Pharmacy Technician at Wegman's Pharmacy, Princeton NJ September 2009 - August 2010

- Claims Representative at Spinal \& Head Trauma Associates, Marlboro, NJ May 2007 - August 2008 